\title{
Z-Enolate Geometry in Thioamide Aldol Reaction Illuminated by 7-Azaindoline Auxiliary
}

\author{
Roman Pluta, Zhao Li, Naoya Kumagai, * and Masakatsu Shibasaki* \\ Institute of Microbial Chemistry (BIKAKEN), Tokyo, Japan \\ mshibasa@bikaken.or.jp, nkumagai@bikaken.or.jp
}

1. General

2. Instrumentation

3. Materials

4. General Procedure

4-1. Preparation of amides

4-2. Preparation of 7-azaindoline thioamides

4-3. Aldol reactions

5. Optimization of Conditions

6. Gram-Scale Reaction

7. Characterization of Products

8. Transformations of Products

9. Crystal Structure Analysis

10. Studies of Copper Complexes

11. References

12. NMR Spectra of New Compounds
S2

$\mathrm{S} 2$

$\mathrm{S} 2$

$\mathrm{S} 2$

$\mathrm{S} 2$

S3

S4

S5

S5

S6-S14

S15

S16-S17

S18-S22

S22

S23-S54 


\section{General}

The catalytic asymmetric aldol reaction of thioamide were performed in a flame-dried $20 \mathrm{~mL}$ glass test tube with a Tefloncoated magnetic stirring bar unless otherwise noted. The flasks or test tubes were fitted with a 3-way glass stopcock and reactions were run under Ar atmosphere. Air- and moisture-sensitive liquids were transferred via a gas-tight syringe and a stainless-steel needle. All work-up and purification procedures were carried out with reagent-grade solvents under ambient atmosphere. An oil bath was used for all reactions needed heating.

\section{Instrumentation}

Infrared (IR) spectra were recorded on a HORIBA FT210 Fourier transform infrared spectrophotometer. NMR was recorded on Bruker AVANCE III HD400 NMR spectrometers. Chemical shifts for proton are reported in parts per million downfield from tetramethylsilane and are referenced to residual protium in the NMR solvent ( $\mathrm{CDCl}_{3}: \delta$ 7.26 ppm). For ${ }^{13} \mathrm{CNMR}$, chemical shifts were reported in the scale relative to NMR solvent $\left(\mathrm{CDCl}_{3}: \delta 77.16 \mathrm{ppm}\right)$ as an internal reference. NMR data are reported as follows: chemical shifts, multiplicity (s: singlet, d: doublet, t: triplet, q: quartet, m: multiplet, br: broad signal), coupling constant $(\mathrm{Hz})$, and integration. Single-crystal X-ray data were collected on a Rigaku R-AXIS RAPID II imaging plate area detector with graphite-monochromated $\mathrm{Cu}-\mathrm{K} \alpha$ radiation. Optical rotation was measured using a $1 \mathrm{~mL}$ cell with a $1.0 \mathrm{dm}$ path length on a JASCO polarimeter P-1030. High-resolution mass spectra (ESI TOF (+)) were measured on Thermo Fisher Scientific LTQ Orbitrap XL. HPLC analysis was conducted on a JASCO HPLC system equipped with Daicel chiral-stationary-phase columns $(\phi 0.46 \mathrm{~cm} \times 25 \mathrm{~cm})$.

\section{Materials}

Unless otherwise noted, materials were purchased from commercial suppliers and were used without further purification. THF was purified by passing through a solvent purification system (Glass Contour). Ligands and $\left[\mathrm{Cu}(\mathrm{MeCN})_{4}\right] \mathrm{PF}_{6}$ were purchased from Strem Chemical, Inc. or Sigma-Aldrich and used as received (opened and handled in a glove box). Amides S2a, S2b were prepared according to previously reported procedures. ${ }^{[1]}$

Column chromatography was performed with silica gel Merck 60 (230-400 mesh ASTM), silica gel 60 N (spherical, neutral, 40-50 $\mathrm{m}$ ) from Kanto Chemical Co., Inc. Preparative TLC plates (1.05744.0001, PLC Silica gel 60 F254) were purchased from Merck.

\section{General procedures}

\section{4-1. Preparation of 7-azaindoline amides}

To a solution of the corresponding carboxylic acid (12 mmol, 1.2 equiv), 2,3-dihydro-7-azaindole (1.20 g, $10 \mathrm{mmol}, 1$ equiv) and DMAP (120 mg, $1.0 \mathrm{mmol}, 10 \mathrm{~mol} \%)$ in dry DCM (50 mL) was added solid EDCI (2.30 g, $12 \mathrm{mmol}, 1.2$ equiv) in one portion and the mixture was stirred at RT overnight. The reaction mixture was diluted with water $(150 \mathrm{~mL})$ and the product was extracted into DCM $(3 \times 50 \mathrm{~mL})$. Combined organic layers were dried over $\mathrm{Na}_{2} \mathrm{SO}_{4}$ and concentrated under reduced pressure. The crude products were purified by silica gel chromatography (ethyl acetate/hexane).

\section{1-(2,3-Dihydro-1H-pyrrolo[2,3-b]pyridin-1-yl)-3-phenylpropan-1-one (S2c)}

The reaction performed according to the general procedure afforded $1.97 \mathrm{~g}(78 \%)$ (ethyl acetate/hexane
$=1 / 3)$. Colorless solid $\left(\mathrm{m} . \mathrm{p} .77-79{ }^{\circ} \mathrm{C}\right) ;{ }^{1} \mathrm{H} \mathrm{NMR}\left(400 \mathrm{MHz}, 300 \mathrm{~K}, \mathrm{CDCl}_{3}\right): \delta 8.14-8.06(\mathrm{~m}, 1 \mathrm{H}), 7.43(\mathrm{ddd}$,
$J=7.3,2.8,1.3 \mathrm{~Hz}, 1 \mathrm{H}), 7.35-7.26(\mathrm{~m}, 4 \mathrm{H}), 7.22-7.15(\mathrm{~m}, 1 \mathrm{H}), 6.85(\mathrm{dd}, J=7.3,5.1 \mathrm{~Hz}, 1 \mathrm{H}), 4.15-4.05(\mathrm{~m}$,
$2 \mathrm{H}), 3.51-3.40(\mathrm{~m}, 2 \mathrm{H}), 3.10-2.98(\mathrm{~m}, 4 \mathrm{H}) ;{ }^{13} \mathrm{C} \mathrm{NMR}\left(101 \mathrm{MHz}, 300 \mathrm{~K}, \mathrm{CDCl}_{3}\right): \delta 172.2,156.0,146.2,141.8$, 133.3, 128.6, 128.3, 126.0, 125.9, 117.9, 45.6, 38.4, 31.1, 24.2; IR (thin film): $\widetilde{v} 3025,2922,1658,1602,1587,1421,1243,782$, 746, $700 \mathrm{~cm}^{-1}$; HRMS (ESI): $\mathrm{m} / \mathrm{z}$ calculated for $\mathrm{C}_{16} \mathrm{H}_{17} \mathrm{~N}_{2} \mathrm{O}^{+}[\mathrm{M}+\mathrm{H}]^{+}: 253.1335$, found: 253.1340 . 


\section{3-(Benzyloxy)-1-(2,3-dihydro-1H-pyrrolo[2,3-b]pyridin-1-yl)propane-1-one (S2d)}

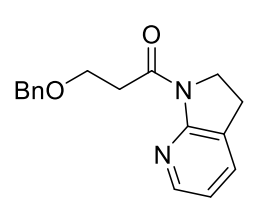

The reaction performed according to the general procedure afforded $1.92 \mathrm{~g}(68 \%)$ (ethyl acetate/hexane = 1/1). Colorless oil; ${ }^{1} \mathrm{H}$ NMR (400 MHz, $\left.300 \mathrm{~K}, \mathrm{CDCl}_{3}\right): \delta 8.09$ (ddt, J= 5.1, 1.8, $\left.1.1 \mathrm{~Hz}, 1 \mathrm{H}\right), 7.43$ (ddt, J = 7.3, 1.7, 1.1 Hz, 1H), 7.37-7.28 (m, 4H), 7.28-7.22 (m, 1H), $6.85(\mathrm{dd}, J=7.3,5.1 \mathrm{~Hz}, 1 \mathrm{H}), 4.58(\mathrm{~s}, 2 \mathrm{H})$, 4.14-4.07 (m, 2H), $3.91(\mathrm{t}, J=6.6 \mathrm{~Hz}, 2 \mathrm{H}), 3.51(\mathrm{t}, J=6.6 \mathrm{~Hz}, 2 \mathrm{H}), 3.07-3.00(\mathrm{~m}, 2 \mathrm{H}) ;{ }^{13} \mathrm{C} \mathrm{NMR}(101 \mathrm{MHz}$, $\left.300 \mathrm{~K}_{,} \mathrm{CDCl}_{3}\right): \delta 170.6,156.0,146.1,138.6,133.3,128.3,127.7,127.5,126.0,117.9,73.0,66.0,45.5,37.1,24.2 ; \mathrm{IR}$ (thin film): $\widetilde{v} 2864,1658,1601,1588,1422,1242,1099,785,738,698 \mathrm{~cm}^{-1} ;$ HRMS (ESI): $m / z$ calculated for $\mathrm{C}_{17} \mathrm{H}_{19} \mathrm{~N}_{2} \mathrm{O}_{2}{ }^{+}[\mathrm{M}+\mathrm{H}]^{+}: 283.1441$, found: 283.1449 .

\section{Ethyl 5-(2,3-dihydro-1H-pyrrolo[2,3-b]pyridin-1-yl)-5-oxopentanoate (S2e)}

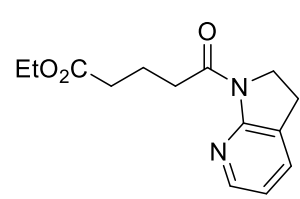

The reaction performed according to the general procedure afforded $1.94 \mathrm{~g}$ (74\%) (ethyl acetate/hexane $=1 / 2)$. Colorless solid (m.p. $\left.74-76^{\circ} \mathrm{C}\right) ;{ }^{1} \mathrm{H} \mathrm{NMR}\left(400 \mathrm{MHz}, 300 \mathrm{~K}, \mathrm{CDCl}_{3}\right): \delta 8.09$ (ddt, $J=5.1,1.6,1.1 \mathrm{~Hz}, 1 \mathrm{H}), 7.45(\mathrm{dq}, J=7.3,1.1 \mathrm{~Hz}, 1 \mathrm{H}), 6.86(\mathrm{dd}, J=7.3,5.1 \mathrm{~Hz}, 1 \mathrm{H}), 4.20-4.03(\mathrm{~m}, 4 \mathrm{H})$, $3.19(\mathrm{t}, J=7.3 \mathrm{~Hz}, 2 \mathrm{H}), 3.10-3.01(\mathrm{~m}, 2 \mathrm{H}), 2.45(\mathrm{t}, J=7.5 \mathrm{~Hz}, 2 \mathrm{H}), 2.06(\mathrm{p}, J=7.4 \mathrm{~Hz}, 2 \mathrm{H}), 1.25(\mathrm{t}, J=$ $7.1 \mathrm{~Hz}, 3 \mathrm{H}) ;{ }^{13} \mathrm{C}$ NMR (101 MHz, $\left.300 \mathrm{~K}, \mathrm{CDCl}_{3}\right)$ : $\delta$ 173.5, 172.3, 156.0, 146.1, 133.3, 126.0, 117.9, 60.2, 45.6, 35.7, 33.8, 24.2, 20.3, 14.3; IR (thin film): $\widetilde{v} 2977,1732,1659,1602,1588,1421,1307,1241,1029,784 \mathrm{~cm}^{-1}$; HRMS (ESI): $m / z$ calculated for $\mathrm{C}_{14} \mathrm{H}_{19} \mathrm{~N}_{2} \mathrm{O}_{3}{ }^{+}[\mathrm{M}+\mathrm{H}]^{+}:$263.1390, found: 263.1394 .

\section{1-(2,3-Dihydro-1H-pyrrolo[2,3-b]pyridin-1-yl)-4,4,4-trifluorobutane-1-one (S2f)}

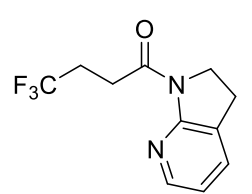

The reaction performed according to the general procedure afforded $1.71 \mathrm{~g}(70 \%)$. (ethyl acetate/hexane = 1/3). Colorless solid (m.p. 64-66 $\left.{ }^{\circ} \mathrm{C}\right) ;{ }^{1} \mathrm{H}$ NMR (400 MHz, $\left.300 \mathrm{~K}, \mathrm{CDCl}_{3}\right): \delta 8.12$ (ddt, J= 5.1, 1.7, $1.1 \mathrm{~Hz}$, $1 \mathrm{H}), 7.48(\mathrm{ddd}, J=7.4,2.9,1.1 \mathrm{~Hz}, 1 \mathrm{H}), 6.90(\mathrm{dd}, J=7.4,5.1 \mathrm{~Hz}, 1 \mathrm{H}), 4.17-4.04(\mathrm{~m}, 2 \mathrm{H}), 3.52-3.35(\mathrm{~m}, 2 \mathrm{H})$, 3.11-3.03 (m, 2H), 2.68-2.49 (m, 2H); ${ }^{13} \mathrm{C} \mathrm{NMR}\left(101 \mathrm{MHz}, 300 \mathrm{~K}, \mathrm{CDCl}_{3}\right): \delta 169.8,155.8,146.3,133.5,127.3$ $(q, J=276.0 \mathrm{~Hz}), 125.9,118.3,45.7,29.8(q, J=3.0 \mathrm{~Hz}), 29.2(\mathrm{q}, J=29.4 \mathrm{~Hz}), 24.3 ;{ }^{19} \mathrm{~F} \mathrm{NMR}(376 \mathrm{MHz}, 300 \mathrm{~K}, \mathrm{CDCl}): \delta-66.4$ (t, $J=10.9 \mathrm{~Hz}$ ); IR (thin film): $\widetilde{v} 2963,1662,1601,1590,1427,1356,1136,977,785,625 \mathrm{~cm}^{-1}$; HRMS (ESI): $m / z$ calculated for $\mathrm{C}_{11} \mathrm{H}_{12} \mathrm{~N}_{2} \mathrm{OF}_{3}{ }^{+}[\mathrm{M}+\mathrm{H}]^{+}:$245.0896, found: 245.0900 .

\section{4-2. Preparation of 7-azaindoline thioamides}

A solution of the corresponding 7-azaindoline amide $(5 \mathrm{mmol})$ and Lawesson's reagent $(1.36 \mathrm{~g}, 3.4 \mathrm{mmol}, 0.67 \mathrm{equiv})$ in dioxane $(50 \mathrm{~mL})$ was refluxed in an oil bath for 1-4 days (formation of product was monitored by TLC analysis). After cooling to room temereature, the solvent was removd under reduced pressure. The residue was filtered through short pad of silica gel $(5 \mathrm{~cm}$ ) eluting with hexane/ethyl acetate 2/1 to remove Lawesson's reagent byproducts, the filtrates containing the product were concentrated under reduced pressure and purified by flash chromatography on silica gel in hexane/ethyl acetate gradient. The products were obtained as pale yellow solids. If necessary, the thioamides can be recrystallized form dichloromethane/hexane.

\section{1-(2,3-Dihydro-1H-pyrrolo[2,3-b]pyridin-1-yl)propane-1-thione (2a)}

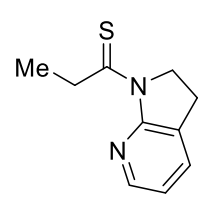

The reaction performed according to the general procedure afforded $625 \mathrm{mg}(65 \%)$. (ethyl acetate/hexane = 1/9). Pale yellow crystals (m.p. $\left.75-77^{\circ} \mathrm{C}\right) ;{ }^{1} \mathrm{H}$ NMR $\left(400 \mathrm{MHz}, 300 \mathrm{~K}, \mathrm{CDCl}_{3}\right): \delta 8.22(\mathrm{ddt}, J=5.0,1.8,1.1$ $\mathrm{Hz}, 1 \mathrm{H}), 7.57(\mathrm{ddd}, J=7.4,2.9,1.1 \mathrm{~Hz}, 1 \mathrm{H}), 6.99(\mathrm{dd}, J=7.4,5.0 \mathrm{~Hz}, 1 \mathrm{H}), 4.56-4.47(\mathrm{~m}, 2 \mathrm{H}), 3.72(\mathrm{q}, J=7.3$

$\mathrm{Hz}, 2 \mathrm{H}), 3.16-3.06(\mathrm{~m}, 2 \mathrm{H}), 1.36(\mathrm{t}, J=7.3 \mathrm{~Hz}, 3 \mathrm{H}) ;{ }^{13} \mathrm{C} \mathrm{NMR}\left(101 \mathrm{MHz}, 300 \mathrm{~K}, \mathrm{CDCl}_{3}\right): \delta 208.4,155.6,146.6$, 134.1, 128.2, 119.3, 53.9, 38.5, 23.9, 14.2; IR (thin film): $\widetilde{v} 2965,2930,1589,1440,1409,1359,1235,1104,946,794 \mathrm{~cm}^{-1} ; \mathrm{HRMS}^{\circ}$ (ESI): $m / z$ calculated for $\mathrm{C}_{10} \mathrm{H}_{13} \mathrm{~N}_{2} \mathrm{~S}^{+}[\mathrm{M}+\mathrm{H}]^{+}:$193.0794, found: 193.0799 .

\section{1-(2,3-Dihydro-1H-pyrrolo[2,3-b]pyridin-1-yl)butane-1-thione (2b)}

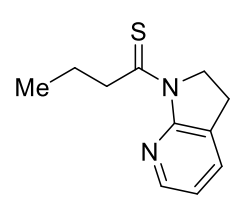

The reaction performed according to the general procedure afforded $675 \mathrm{mg}$ (65\%). (ethyl acetate/hexane $=1 / 9)$. Pale yellow crystals (m.p. $\left.72-74{ }^{\circ} \mathrm{C}\right) ;{ }^{1} \mathrm{H} \mathrm{NMR}\left(400 \mathrm{MHz}, 300 \mathrm{~K}, \mathrm{CDCl}_{3}\right): \delta 8.22(\mathrm{ddt}$, $J=5.0,1.7,1.0 \mathrm{~Hz}, 1 \mathrm{H}), 7.57(\mathrm{ddd}, J=7.4,2.7,1.0 \mathrm{~Hz}, 1 \mathrm{H}), 6.98(\mathrm{dd}, J=7.4,5.0 \mathrm{~Hz}, 1 \mathrm{H}), 4.57-4.45$ (m, $2 \mathrm{H})$, 3.77-3.66 (m, 2H), 3.17-3.04 (m, 2H), 1.88-1.74 (m, 2H), 1.05 (t, J=7.3 Hz, 3H); ${ }^{33} \mathrm{C} \mathrm{NMR}(101 \mathrm{MHz}, 300 \mathrm{~K}$, 

$\mathrm{CDCl}_{3}$ ): $\delta$ 207.0, 155.6, 146.6, 134.1, 128.2, 119.3, 53.8, 47.0, 23.9, 23.4, 13.8; IR (thin film): $\widetilde{v} 2963,1589,1437,1407,1234,791$ $\mathrm{cm}^{-1}$; HRMS (ESI): $m / z$ calculated for $\mathrm{C}_{11} \mathrm{H}_{15} \mathrm{~N}_{2} \mathrm{~S}^{+}[\mathrm{M}+\mathrm{H}]^{+}:$207.0950, found: 207.0952.

\section{1-(2,3-Dihydro-1H-pyrrolo[2,3-b]pyridin-1-yl)-3-phenylpropane-1-thione (2c)}

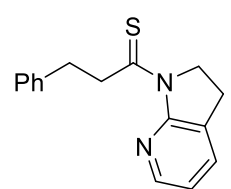

The reaction performed according to the general procedure afforded $835 \mathrm{mg}(62 \%)$.(ethyl acetate/hexane = 1/9). Pale yellow crystals (m.p. $\left.75-77^{\circ} \mathrm{C}\right) ;{ }^{1} \mathrm{H} \mathrm{NMR}\left(400 \mathrm{MHz}, 300 \mathrm{~K}, \mathrm{CDCl}_{3}\right): \delta 8.26(\mathrm{ddt}, J=5.0,1.7,1.1$ $\mathrm{Hz}, 1 \mathrm{H}), 7.57(\mathrm{ddd}, J=7.4,2.8,1.1 \mathrm{~Hz}, 1 \mathrm{H}), 7.39-7.32(\mathrm{~m}, 2 \mathrm{H}), 7.32-7.26(\mathrm{~m}, 2 \mathrm{H}), 7.22-7.14(\mathrm{~m}, 1 \mathrm{H}), 7.00$ $(\mathrm{dd}, J=7.4,5.0 \mathrm{~Hz}, 1 \mathrm{H}), 4.57-4.46(\mathrm{~m}, 2 \mathrm{H}), 4.08-4.00(\mathrm{~m}, 2 \mathrm{H}), 3.15-3.06(\mathrm{~m}, 4 \mathrm{H}) ;{ }^{13} \mathrm{C}$ NMR $(101 \mathrm{MHz}, 300$ $\mathrm{K}, \mathrm{CDCl}_{3}$ ): $\delta$ 205.4, 155.6, 146.6, 141.5, 134.3, 128.7, 128.3, 128.2, 126.0, 119.5, 53.9, 47.1, 36.0, 24.0; IR (thin film): $\widetilde{v} 3024$, 2947, 1592, 1432, 1412, 1243, 1131, 785, $699 \mathrm{~cm}^{-1}$; HRMS (ESI): $\mathrm{m} / z$ calculated for $\mathrm{C}_{16} \mathrm{H}_{17} \mathrm{~N}_{2} \mathrm{~S}^{+}[\mathrm{M}+\mathrm{H}]^{+}: 269.1107$, found: 269.1113.

\section{3-(Benzyloxy)-1-(2,3-dihydro-1H-pyrrolo[2,3-b]pyridin-1-yl)propane-1-thione (2d)}

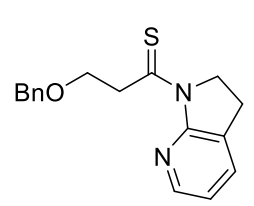

The reaction performed according to the general procedure afforded $690 \mathrm{mg}$ (64\%). (ethyl acetate/hexane =1/5). Pale yellow crystals (m.p. $\left.66-68^{\circ} \mathrm{C}\right) ;{ }^{1} \mathrm{H} \mathrm{NMR}\left(400 \mathrm{MHz}, 300 \mathrm{~K}, \mathrm{CDCl}_{3}\right): \delta 8.19$ $(\mathrm{ddt}, J=5.0,1.8,1.1 \mathrm{~Hz}, 1 \mathrm{H}), 7.55(\mathrm{ddd}, J=7.4,2.9,1.1 \mathrm{~Hz}, 1 \mathrm{H}), 7.37-7.28(\mathrm{~m}, 4 \mathrm{H}), 7.27-7.22(\mathrm{~m}, 1 \mathrm{H}), 6.98$ $(\mathrm{dd}, \mathrm{J}=7.4,5.0 \mathrm{~Hz}, 1 \mathrm{H}), 4.60(\mathrm{~s}, 2 \mathrm{H}), 4.54-4.44(\mathrm{~m}, 2 \mathrm{H}), 4.14-4.07(\mathrm{~m}, 2 \mathrm{H}), 4.03-3.97(\mathrm{~m}, 2 \mathrm{H}), 3.12-3.05(\mathrm{~m}$, 2H); ${ }^{13} \mathrm{C}$ NMR (101 MHz, $\left.300 \mathrm{~K}, \mathrm{CDCl}_{3}\right): \delta$ 202.2, 155.7, 146.5, 138.7, 134.2, 128.3, 128.2, 127.7, 127.4, 119.5, 72.9, 69.6, 53.8, 44.5, 23.9; IR (thin film): $\widetilde{v} 3028,2860,1591,1413,1246,1099,786,737,698 \mathrm{~cm}^{-1}$; HRMS (ESI): $\mathrm{m} / z$ calculated for $\mathrm{C}_{17} \mathrm{H}_{19} \mathrm{~N}_{2} \mathrm{OS}^{+}[\mathrm{M}+\mathrm{H}]^{+}: 299.1213$, found: 299.1219 .

\section{Ethyl 5-(2,3-dihydro-1H-pyrrolo[2,3-b]pyridin-1-yl)-5-thioxopentanoate (2e)}

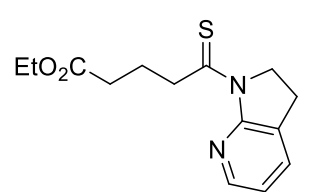

The reaction performed according to the general procedure afforded $710 \mathrm{mg}$ (51\%). (ethyl acetate/hexane =1/9). Pale yellow crystals (m.p. $\left.57-59^{\circ} \mathrm{C}\right) ;{ }^{1} \mathrm{H} \mathrm{NMR}\left(400 \mathrm{MHz}, 300 \mathrm{~K}, \mathrm{CDCl}_{3}\right): \delta 8.23-$ $8.18(\mathrm{~m}, 1 \mathrm{H}), 7.60-7.55(\mathrm{~m}, 1 \mathrm{H}), 6.99(\mathrm{dd}, J=7.4,5.0 \mathrm{~Hz}, 1 \mathrm{H}), 4.55-4.43(\mathrm{~m}, 2 \mathrm{H}), 4.12(\mathrm{q}, J=7.1 \mathrm{~Hz}$, $2 \mathrm{H}), 3.83-3.67(\mathrm{~m}, 2 \mathrm{H}), 3.17-3.05(\mathrm{~m}, 2 \mathrm{H}), 2.55-2.41(\mathrm{~m}, 2 \mathrm{H}), 2.21-2.05(\mathrm{~m}, 2 \mathrm{H}), 1.25(\mathrm{t}, J=7.1 \mathrm{~Hz}, 3 \mathrm{H})$; ${ }^{13} \mathrm{C}$ NMR (101 MHz, $\left.300 \mathrm{~K}, \mathrm{CDCl}_{3}\right): \delta$ 205.2, 173.5, 155.6, 146.6, 134.2, 128.2, 119.4, 60.2, 53.8, 44.1, 33.7, 24.8, 23.9, 14.3; IR (thin film): $\widetilde{v} 2979,1726,1590,1412,1245,787 \mathrm{~cm}^{-1} ; \mathrm{HRMS}$ (ESI): $\mathrm{m} / z$ calculated for $\mathrm{C}_{14} \mathrm{H}_{19} \mathrm{~N}_{2} \mathrm{O}_{2} \mathrm{~S}^{+}[\mathrm{M}+\mathrm{H}]^{+}: 279.1162$, found: 279.1166.

\section{1-(2,3-Dihydro-1H-pyrrolo[2,3-b]pyridin-1-yl)-4,4,4-trifluorobutane-1-thione (2f)}

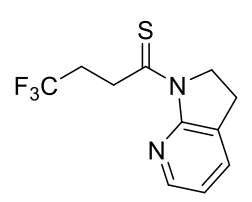

The reaction performed according to the general procedure afforded $720 \mathrm{mg}$ (55\%). (ethyl acetate/hexane $=1 / 10)$. Pale yellow crystals (m.p. 90-92 $\left.{ }^{\circ} \mathrm{C}\right) ;{ }^{1} \mathrm{H}$ NMR $\left(400 \mathrm{MHz}, 300 \mathrm{~K}, \mathrm{CDCl}_{3}\right): \delta 8.22$ $(\mathrm{ddt}, J=5.0,1.8,1.1 \mathrm{~Hz}, 1 \mathrm{H}), 7.60(\mathrm{ddd}, J=7.4,2.8,1.1 \mathrm{~Hz}, 1 \mathrm{H}), 7.03(\mathrm{dd}, J=7.4,5.0 \mathrm{~Hz}, 1 \mathrm{H}), 4.55-4.45$ $(\mathrm{m}, 2 \mathrm{H}), 3.97-3.87(\mathrm{~m}, 2 \mathrm{H}), 3.20-3.10(\mathrm{~m}, 2 \mathrm{H}), 2.78-2.62(\mathrm{~m}, 2 \mathrm{H}) ;{ }^{13} \mathrm{C} \mathrm{NMR}\left(101 \mathrm{MHz}, 300 \mathrm{~K}, \mathrm{CDCl}_{3}\right): \delta$ 201.5, 155.5, 146.7, 134.4, 128.2, 127.0 (q, $J=276.8 \mathrm{~Hz}), 119.8,54.0,37.3(\mathrm{q}, J=3.0 \mathrm{~Hz}), 33.6$ (q, $J=28.7 \mathrm{~Hz}), 24.0 ;{ }^{19} \mathrm{~F}$ NMR (376 MHz, $\left.300 \mathrm{~K}, \mathrm{CDCl}_{3}\right): \delta-66.10$ (t, J = $11.0 \mathrm{~Hz}$ ); IR (thin film): $\widetilde{v} 2978$ 1590, 1421, 1375, 1243, 1124, 1093, 965, $792 \mathrm{~cm}^{-1}$; HRMS (ESI): $m / z$ calculated for $\mathrm{C}_{11} \mathrm{H}_{12} \mathrm{~N}_{2} \mathrm{~F}_{3} \mathrm{~S}^{+}[\mathrm{M}+\mathrm{H}]^{+}: 261.0668$, found: 261.0673 .

\section{4-3. Aldol reactions}

\section{Procedure A:}

A flame-dried $20 \mathrm{~mL}$ test tube equipped with a magnetic stirring bar and a 3-way glass stopcock were charged with thioamide $2(0.1 \mathrm{mmol}),(S, S)-\mathrm{Ph}-\mathrm{BPE}(3.05 \mathrm{mg}, 6.0 \mu \mathrm{mol}, 6 \mathrm{~mol} \%)$ and $\left[\mathrm{Cu}(\mathrm{MeCN})_{4}\right] \mathrm{PF}_{6}(1.9 \mathrm{mg}, 5.0 \mu \mathrm{mol}, 5 \mathrm{~mol} \%)$ in a glove box. To the mixture was added anhydrous THF $(0.85 \mathrm{~mL})$ via syringe with a stainless-steel needle at room temperature. The resulting solution was stirred for $15 \mathrm{~min}$ and transferred to cooling bath kept at $-60{ }^{\circ} \mathrm{C}$. Aldehyde $(0.2 \mathrm{mmol})$ was added by syringe followed by a solution of lithium phenoxide $(0.15 \mathrm{~mL}, 0.1 \mathrm{M}$ in THF, $15.0 \mu \mathrm{mol}, 15$ mol\%). After $48 \mathrm{~h}$ of stirring at $-60^{\circ} \mathrm{C}$, the reaction mixture was quenched with a solution of acetic acid $(0.1 \mathrm{M}$ in THF $1.0 \mathrm{~mL}, 0.1 \mathrm{mmol}$ ). After evaporation of the solvent under reduced pressure, the residue was purified by silica gel chromatography. The enantiomeric excess (ee) was determined by HPLC analysis. The diastereomeric ratio (d.r.) was 
determined by ${ }^{1} \mathrm{HNMR}$ spectroscopy of the crude product.

\section{Procedure B:}

Following the above Procedure A, however reaction was conducted in $-78^{\circ} \mathrm{C}$ for $96 \mathrm{~h}$ before addition of a solution of acetic acid.

\section{Optimization of Conditions}
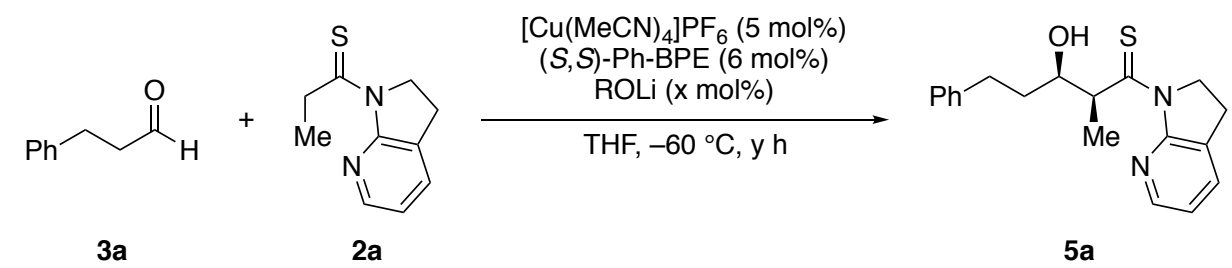

\begin{tabular}{|c|c|c|c|c|c|c|}
\hline & & a & & & & $5 a$ \\
\hline entry & ROLi & x (mol\%) & $y(h)$ & yield of $5 \mathbf{a}(\%)^{a}$ & $d r($ syn/anti) & ee of syn (\%) \\
\hline 1 & $p-\mathrm{MeOC}_{6} \mathrm{H}_{4} \mathrm{OLi}$ & 5 & 48 & 49 & $>20 / 1$ & 95 \\
\hline 2 & ${ }^{t} \mathrm{BuOLi}$ & 5 & 48 & 71 & $6 / 1$ & 20 \\
\hline 3 & PhOLi & 5 & 48 & 52 & $>20 / 1$ & 95 \\
\hline 4 & PhOLi & 5 & 96 & 68 & $>20 / 1$ & 95 \\
\hline 5 & PhOLi & 15 & 48 & $71^{b}$ & $>20 / 1$ & 95 \\
\hline
\end{tabular}

\section{Aldol reactions using Mesitylcopper}

A flame-dried 20-mL test tube equipped with a magnetic stir bar and a 3-way glass stopcock was charged with thioamide 2a $(19.2 \mathrm{mg}, 0.1 \mathrm{mmol})$. THF $(0.9 \mathrm{~mL})$ was added to the mixture and the test tube was immersed into an electronicallycontrolled cooling bath at $-60^{\circ} \mathrm{C}$. The catalyst solution was prepared as follows and added to the solution. A flame-dried 20-mL test tube equipped with a magnetic stir bar and a 3-way glass stopcock was charged with (S,S)-Ph-BPE (3.05 mg, $6.0 \mu \mathrm{mol}, 6 \mathrm{~mol} \%)$ and mesitylcopper $(0.9 \mathrm{mg}, 5.0 \mu \mathrm{mol}, 5 \mathrm{~mol} \%)$ in a glove box. THF $(0.1 \mathrm{~mL})$ was added to the mixture via a syringe equipped with a stainless-steel needle at room temperature. A pale-yellow solution of $(S, S)-\mathrm{Ph}$ $\mathrm{BPE} / \mathrm{mesitylcopper}$ was obtained, which was used within $5 \mathrm{~min}$. After the addition of and aldehyde $3 \mathrm{a}(0.2 \mathrm{mmol})$ at $-60{ }^{\circ} \mathrm{C}$, the mixture was stirred for $48 \mathrm{~h}$ at the same temperature. The reaction was quenched with a solution of acetic acid $(0.1 \mathrm{M}$ in THF $1.0 \mathrm{~mL}, 0.1 \mathrm{mmol})$ and then evaporated under reduced pressure before analyzed.

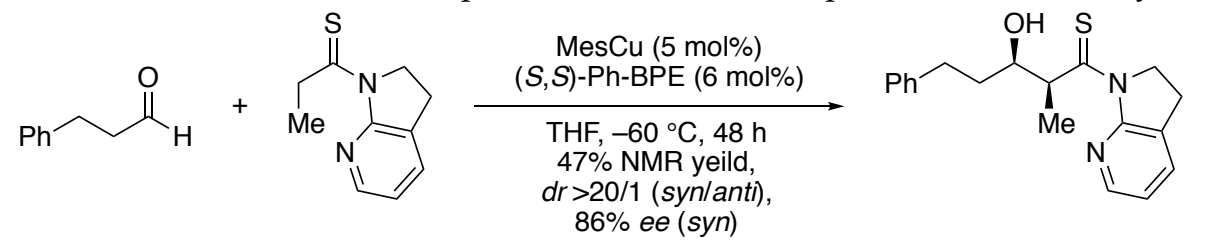

\section{Gram-Scale Reaction}

A flame-dried $100 \mathrm{~mL}$ flask equipped with a magnetic stirring bar and a 3-way glass stopcock were charged with 2a (0.96 g, $5 \mathrm{mmol}),(S, S)-\mathrm{Ph}-\mathrm{BPE}(152 \mathrm{mg}, 0.3 \mathrm{mmol}, 6 \mathrm{~mol} \%)$ and $\left[\mathrm{Cu}(\mathrm{MeCN})_{4}\right] \mathrm{PF}_{6}(93 \mathrm{mg}, 0.25 \mathrm{mmol}, 5 \mathrm{~mol} \%)$ in a glove box. To the mixture was added anhydrous THF $(49 \mathrm{~mL})$ via syringe with a stainless-steel needle at room temperature. The resulting solution was stirred for $5 \mathrm{~min}$ and transferred to cooling bath kept at $-60{ }^{\circ} \mathrm{C}$. 3-Phenylpropionaldehyde $3 \mathbf{a}$ $(1.32 \mathrm{~mL}, 1.34 \mathrm{~g}, 10 \mathrm{mmol}, 2$ equiv) was added by syringe followed by a solution of lithium phenoxide $(0.75 \mathrm{~mL}, 1 \mathrm{M}$ in THF, $0.75 \mathrm{mmol}, 15 \mathrm{~mol} \%$ ). After $48 \mathrm{~h}$ of stirring at $-60^{\circ} \mathrm{C}$, the reaction mixture was quenched with a solution of acetic acid (10 mL, 0.5 M in THF). After evaporation of volatiles under reduced pressure, the residue was purified by silica gel chromatography (ethyl acetate/hexane $=2 / 3$ ) giving product $5 \mathrm{a}(1.13 \mathrm{~g}$, 69\%). The enantiomeric excess (ee) was determined by HPLC analysis (94\%). The diastereomeric ratio (d.r.) was determined by ${ }^{1}$ HNMR spectroscopy of the crude product (d.r. $>20: 1)$. 


\section{Characterization of Products}

\section{(2S,3R)-1-(2,3-Dihydro-1H-pyrrolo[2,3-b]pyridin-1-yl)-3-hydroxy-2-methyl-5-phenylpentane-1-thione (5a)}

Reaction performed according to procedure $A$ gave after purification product 5a $(23.3 \mathrm{mg}, 71 \%$, d.r. $>20: 1)$ (ethyl acetate/hexane $=2 / 3$ ). Reaction performed according to Gram-Scale Reaction gave after purification product $5 \mathbf{a}(1.13 \mathrm{~g}$, $69 \%$, d.r. $>20: 1$ ) (ethyl acetate/hexane $=2 / 3$ ). The diastereomeric ratio (d.r.) was determined by NMR analysis .

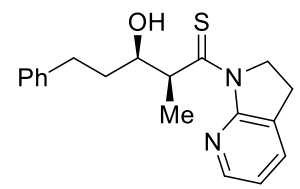

Pale yellow oil; ${ }^{1} \mathrm{H}$ NMR (400 MHz, $\left.300 \mathrm{~K}, \mathrm{CDCl}_{3}\right): \delta 8.17$ (ddt, $\left.J=5.0,1.6,1.1 \mathrm{~Hz}, 1 \mathrm{H}\right), 7.59$ (ddd, $J$ $=7.4,2.8,1.1 \mathrm{~Hz}, 1 \mathrm{H}), 7.30-7.20(\mathrm{~m}, 4 \mathrm{H}), 7.19-7.12(\mathrm{~m}, 1 \mathrm{H}), 7.01(\mathrm{dd}, J=7.4,5.0 \mathrm{~Hz}, 1 \mathrm{H}), 5.28(\mathrm{qd}, J=$ 6.8, $1.7 \mathrm{~Hz}, 1 \mathrm{H}), 4.59-4.44(\mathrm{~m}, 2 \mathrm{H}), 4.33(\mathrm{~s}, 1 \mathrm{H}), 4.06-3.97(\mathrm{~m}, 1 \mathrm{H}), 3.11(\mathrm{t}, J=8.2 \mathrm{~Hz}, 2 \mathrm{H}), 2.90(\mathrm{ddd}$, $J=13.9,10.3,5.3 \mathrm{~Hz}, 1 \mathrm{H}), 2.71(\mathrm{ddd}, J=13.9,10.0,6.5 \mathrm{~Hz}, 1 \mathrm{H}), 2.05-1.93(\mathrm{~m}, 1 \mathrm{H}), 1.81-1.68(\mathrm{~m}, 1 \mathrm{H})$, $1.31(\mathrm{~d}, J=6.8 \mathrm{~Hz}, 3 \mathrm{H}) ;{ }^{13} \mathrm{C}$ NMR $\left(101 \mathrm{MHz}, 300 \mathrm{~K}, \mathrm{CDCl}_{3}\right): \delta 212.1,155.6,146.4,142.6,134.5,128.7,128.5,128.3,125.6$, 119.9, 72.6, 53.8, 46.4, 36.6, 32.5, 23.8, 14.3; IR (thin film): $\widetilde{v} 3399,2934,2863,1592,1413,1313,1240,788,700 \mathrm{~cm}^{-1}$; HRMS (ESI): $m / z$ calculated for $\mathrm{C}_{19} \mathrm{H}_{22} \mathrm{~N}_{2} \mathrm{OSNa}^{+}[\mathrm{M}+\mathrm{Na}]^{+}: 349.1345$, found: $349.1348 ;[\alpha]_{\mathrm{D}}^{26} 5.3\left(c 1.0, \mathrm{CHCl}_{3}, 95 \%\right.$ ee sample).

Enantiomeric excess was determined to be $95 \%$ ee (procedure A) and enantiomeric excess was determined to be $94 \%$ ee (Gram-Scale Reaction), by chiral stationary phase HPLC analysis (CHIRALPAKIG3 $(\phi 0.46 \mathrm{~cm} \times 25 \mathrm{~cm}), n$ hexane/ethanol =90/10, flow rate $1.0 \mathrm{~mL} / \mathrm{min}$, detection at $254 \mathrm{~nm}, \mathrm{t}_{\mathrm{R}}=20.3 \mathrm{~min}$ (major), $23.9 \mathrm{~min}$ (minor), $27.2 \mathrm{~min}$ (anti), $31.2 \min ($ anti).
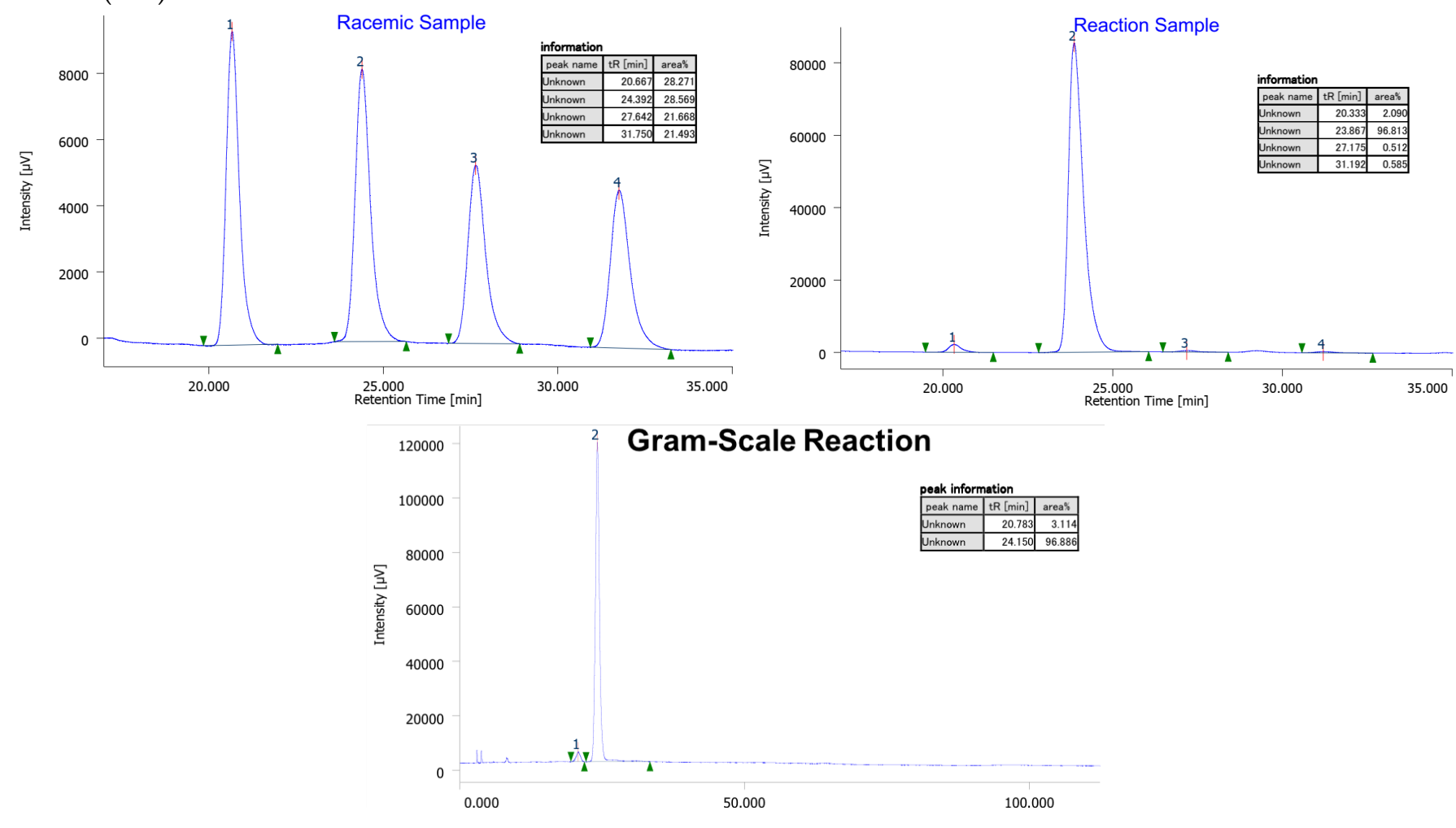

\section{(2S,3R)-1-(2,3-Dihydro-1H-pyrrolo[2,3-b]pyridin-1-yl)-3-hydroxy-2-methyldecane-1-thione (5b)}

Reaction performed according to procedure $A$ gave after purification product $5 \mathbf{b}(25.1 \mathrm{mg}, 78 \%$, d.r. $>20: 1)$ (ethyl acetate/hexane $=2 / 3$ ). The diastereomeric ratio (d.r.) was determined by NMR analysis.

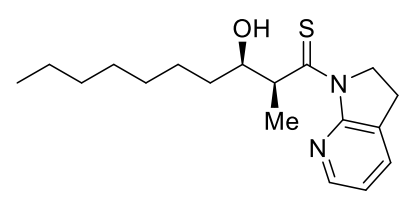

Pale yellow oil; ${ }^{1} \mathrm{H}$ NMR (400 MHz, $\left.300 \mathrm{~K}, \mathrm{CDCl}_{3}\right): \delta 8.20$ (ddt, $\left.J=4.9,1.7,1.1 \mathrm{~Hz}, 1 \mathrm{H}\right), 7.60$ $(\mathrm{ddd}, J=7.5,2.9,1.1 \mathrm{~Hz}, 1 \mathrm{H}), 7.02(\mathrm{dd}, J=7.4,5.0 \mathrm{~Hz}, 1 \mathrm{H}), 5.30(\mathrm{qd}, J=6.8,1.7 \mathrm{~Hz}, 1 \mathrm{H}), 4.62-$ $4.45(\mathrm{~m}, 2 \mathrm{H}), 4.25(\mathrm{~s}, 1 \mathrm{H}), 4.00-3.93(\mathrm{~m}, 1 \mathrm{H}), 3.12(\mathrm{t}, J=8.2 \mathrm{~Hz}, 2 \mathrm{H}), 1.71-1.58(\mathrm{~m}, 1 \mathrm{H}), 1.56-$

$1.42(\mathrm{~m}, 2 \mathrm{H}), 1.41-1.19(\mathrm{~m}, 12 \mathrm{H}), 0.87(\mathrm{t}, J=7.0 \mathrm{~Hz}, 3 \mathrm{H}) ;{ }^{13} \mathrm{C} \mathrm{NMR}\left(101 \mathrm{MHz}, 300 \mathrm{~K}, \mathrm{CDCl}_{3}\right): \delta$ 212.5, 155.6, 146.3, 134.5, 128.7, 119.9, 73.2, 53.9, 46.1, 34.6, 31.9, 29.7, 29.3, 26.1, 23.9, 22.7, 14.1, 14.1; IR (thin film): $\widetilde{v} 3404$, 2927, 2854, 1591, 1413, 1312, 1241, 958, $786 \mathrm{~cm}^{-1}$; HRMS (ESI): $\mathrm{m} / z$ calculated for $\mathrm{C}_{18} \mathrm{H}_{28} \mathrm{~N}_{2} \mathrm{OSNa}^{+}[\mathrm{M}+\mathrm{Na}]^{+}: 343.1815$, found: 
343.1818; $[\alpha]_{\mathrm{D}}^{26}-1.0$ (c 1.0, $\mathrm{CHCl}_{3}, 94 \%$ ee sample).

Enantiomeric excess was determined to be $94 \%$ ee (procedure A), by chiral stationary phase HPLC analysis (CHIRALPAK IA3 $(\phi 0.46 \mathrm{~cm} \times 25 \mathrm{~cm}), n$-hexane $/ 2$-propanol $=95 / 5$, flow rate $1.0 \mathrm{~mL} / \mathrm{min}$, detection at $254 \mathrm{~nm}, \mathrm{t}_{\mathrm{R}}=12.2$ $\min$ (major), $13.6 \mathrm{~min}$ (minor).
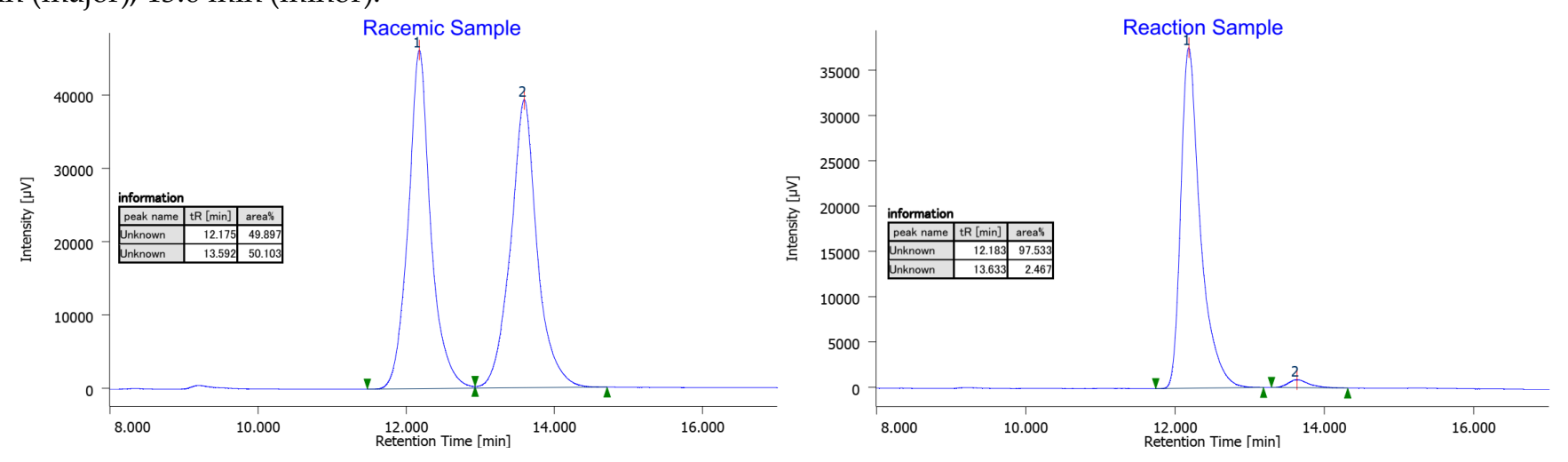

(2S,3R)-1-(2,3-Dihydro-1H-pyrrolo[2,3-b]pyridin-1-yl)-3-hydroxy-2,5-dimethylhexane-1-thione (5c)

Reaction performed according to procedure $A$ gave after purification product 5 c $(20.8 \mathrm{mg}, 74 \%$, d.r. $>20 / 1)$ (ethyl acetate/hexane $=2 / 3$ ). The diastereomeric ratio (d.r.) was determined by NMR analysis.

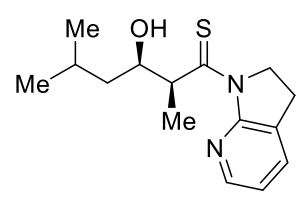

Pale yellow oil; ${ }^{1} \mathrm{H}$ NMR (400 MHz, $\left.300 \mathrm{~K}, \mathrm{CDCl}_{3}\right)$ : $\delta$ 8.24-8.17 (m, 1H), 7.64-7.57 (m, 1H), 7.03 (dd, $J=7.4,5.0 \mathrm{~Hz}, 1 \mathrm{H}), 5.22(\mathrm{qd}, J=6.7,1.3 \mathrm{~Hz}, 1 \mathrm{H}), 4.62-4.44(\mathrm{~m}, 2 \mathrm{H}), 4.18(\mathrm{~s}, 1 \mathrm{H}), 4.13-4.03(\mathrm{~m}, 1 \mathrm{H})$, $3.13(\mathrm{t}, J=8.1 \mathrm{~Hz}, 2 \mathrm{H}), 1.95-1.79(\mathrm{~m}, 1 \mathrm{H}), 1.61(\mathrm{ddd}, J=14.1,9.2,5.3 \mathrm{~Hz}, 1 \mathrm{H}), 1.29(\mathrm{~d}, J=6.8 \mathrm{~Hz}, 3 \mathrm{H})$,

1.27-1.18 (m, 1H), $0.94(\mathrm{~d}, J=3.6 \mathrm{~Hz}, 3 \mathrm{H}), 0.92(\mathrm{~d}, J=3.5 \mathrm{~Hz}, 3 \mathrm{H}) ;{ }^{13} \mathrm{C} \mathrm{NMR}\left(101 \mathrm{MHz}, 300 \mathrm{~K}, \mathrm{CDCl}_{3}\right):$

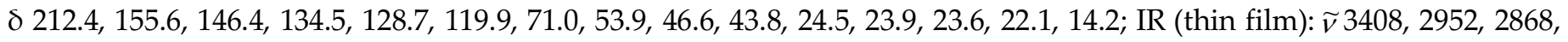
1591, 1413, 1311, 1240, 964, $786 \mathrm{~cm}^{-1}$; HRMS (ESI): $\mathrm{m} / z$ calculated for $\mathrm{C}_{15} \mathrm{H}_{22} \mathrm{~N}_{2} \mathrm{OSNa}{ }^{+}[\mathrm{M}+\mathrm{Na}]^{+}: 301.1435$, found: 301.1435; $[\alpha]_{\mathrm{D}}^{26}-7.6\left(c 1.0, \mathrm{CHCl}_{3}, 96 \%\right.$ ee sample).

Enantiomeric excess was determined to be $96 \%$ ee (procedure A), by chiral stationary phase HPLC analysis (CHIRALPAK IG3 $(\phi 0.46 \mathrm{~cm} \times 25 \mathrm{~cm}), n$-hexane/2-propanol =90/10, flow rate $1.0 \mathrm{~mL} / \mathrm{min}$, detection at $254 \mathrm{~nm}, \mathrm{tr}_{\mathrm{R}}=18.1$ $\min$ (major), $21.2 \mathrm{~min}$ (minor).
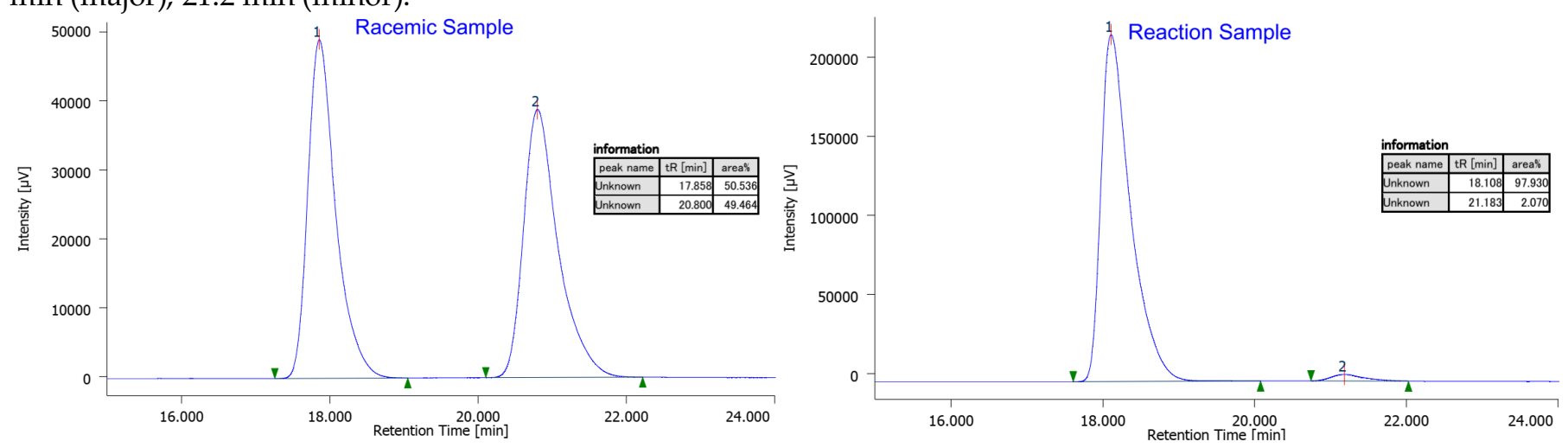

(2S,3R)-1-(2,3-Dihydro-1H-pyrrolo[2,3-b]pyridin-1-yl)-3-hydroxy-2-methyltridec-12-ene-1-thione (5d)

Reaction performed according to procedure A gave after purification product 5d (25.0 mg, 69\%, d.r. >20:1) (ethyl acetate/hexane $=1 / 1)$. The diastereomeric ratio (d.r.) was determined by NMR analysis.

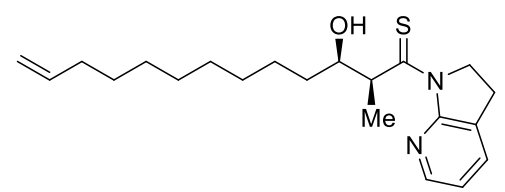

Pale yellow oil; ${ }^{1} \mathrm{H}$ NMR $\left(400 \mathrm{MHz}, 300 \mathrm{~K}, \mathrm{CDCl}_{3}\right): \delta 8.20$ (ddt, $\left.J=5.0,1.8,1.1 \mathrm{~Hz}, 1 \mathrm{H}\right)$, $7.60(\mathrm{dq}, J=7.4,1.1 \mathrm{~Hz}, 1 \mathrm{H}), 7.02(\mathrm{dd}, J=7.4,5.0 \mathrm{~Hz}, 1 \mathrm{H}), 5.81(\mathrm{ddt}, J=16.9,10.2,6.7$ $\mathrm{Hz}, 1 \mathrm{H}), 5.30(\mathrm{qd}, J=6.8,1.6 \mathrm{~Hz}, 1 \mathrm{H}), 5.03-4.87(\mathrm{~m}, 2 \mathrm{H}), 4.61-4.44(\mathrm{~m}, 2 \mathrm{H}), 4.26(\mathrm{~s}, 1 \mathrm{H})$, 4.00-3.92 (m, $1 \mathrm{H}), 3.12(\mathrm{t}, J=8.2 \mathrm{~Hz}, 2 \mathrm{H}), 2.08-1.98(\mathrm{~m}, 2 \mathrm{H}), 1.70-1.58(\mathrm{~m}, 1 \mathrm{H}), 1.55-1.41$ (m, 2H), 1.41-1.22 (m, 14H); ${ }^{13} \mathrm{C}$ NMR (101 MHz, $\left.300 \mathrm{~K}, \mathrm{CDCl}_{3}\right): \delta$ 212.4, 155.6, 146.3, 139.3, 134.5, 128.7, 119.9, 114.1, 73.1, 

53.9, 46.1, 34.6, 33.8, 29.7, 29.6, 29.5, 29.1, 28.9, 26.1, 23.8, 14.1; IR (thin film): $\widetilde{v} 3406,2925,2853,1639,1591,1312,1241,909$, $786 \mathrm{~cm}^{-1}$; HRMS (ESI): $\mathrm{m} / z$ calculated for $\mathrm{C}_{21} \mathrm{H}_{32} \mathrm{~N}_{2} \mathrm{OSNa}^{+}[\mathrm{M}+\mathrm{Na}]^{+}: 383.2128$, found: $383.2127 ;[\alpha]_{\mathrm{D}}^{26}-1.7\left(c 1.0, \mathrm{CHCl}_{3}\right.$, $94 \%$ ee sample).

Enantiomeric excess was determined to be $94 \%$ ee (procedure $A$ ), by chiral stationary phase HPLC analysis $\left(\right.$ CHIRALPAK IG3 $(\phi 0.46 \mathrm{~cm} \times 25 \mathrm{~cm}), n$-hexane/2-propanol =90/10, flow rate $1.0 \mathrm{~mL} / \mathrm{min}$, detection at $254 \mathrm{~nm}, \mathrm{t}_{\mathrm{R}}=18.2$ $\min$ (major), $22.1 \mathrm{~min}$ (minor).
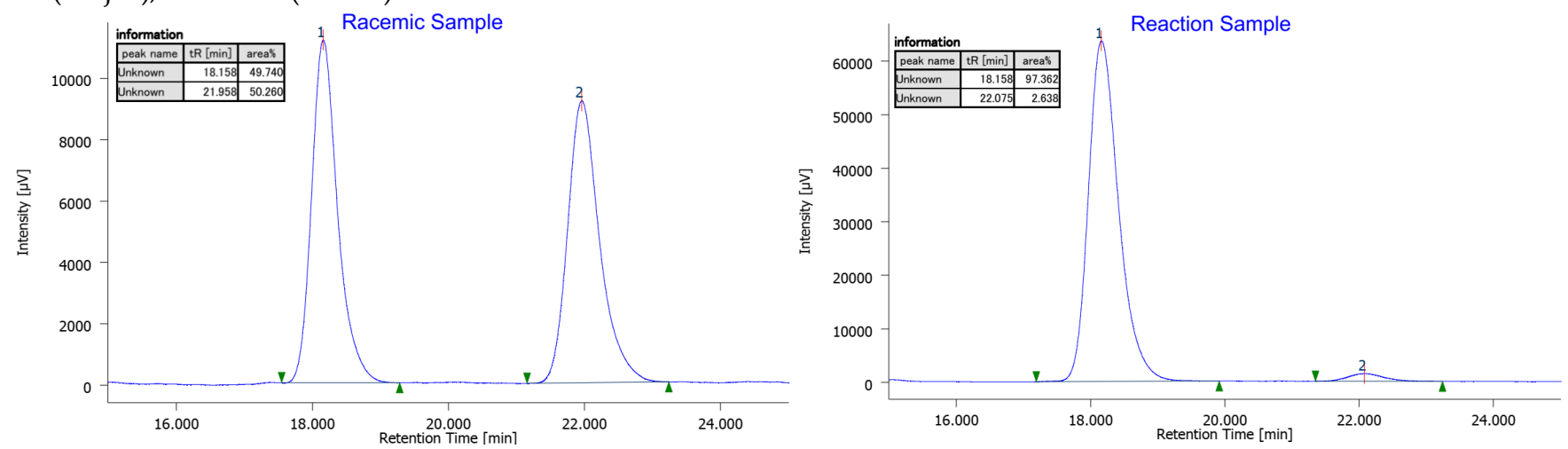

(2S,3R)-1-(2,3-Dihydro-1H-pyrrolo[2,3-b]pyridin-1-yl)-3-hydroxy-2-methyl-5-(trityloxy)pentane-1-thione (5e)

Reaction performed according to procedure A gave after purification product 5e (39.2 mg, 77\%, d.r. >20:1) (ethyl acetate/hexane $=1 / 4$ ). The diastereomeric ratio (d.r.) was determined by NMR analysis.

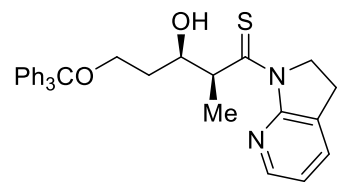

Pale yellow oil; ${ }^{1} \mathrm{H}$ NMR (400 MHz, $\left.300 \mathrm{~K}, \mathrm{CDCl}_{3}\right): \delta 8.07$ (ddt, J=4.9, 1.7, $\left.1.2 \mathrm{~Hz}, 1 \mathrm{H}\right), 7.58$ (dq, $J=7.5,1.2 \mathrm{~Hz}, 1 \mathrm{H}), 7.44-7.39(\mathrm{~m}, 6 \mathrm{H}), 7.28-7.21(\mathrm{~m}, 6 \mathrm{H}), 7.21-7.15(\mathrm{~m}, 3 \mathrm{H}), 6.99(\mathrm{dd}, J=7.5,4.9 \mathrm{~Hz}$, $1 \mathrm{H}), 5.25(\mathrm{qd}, J=6.7,2.7 \mathrm{~Hz}, 1 \mathrm{H}), 4.59-4.43(\mathrm{~m}, 2 \mathrm{H}), 4.24-4.16(\mathrm{~m}, 1 \mathrm{H}), 4.12(\mathrm{~s}, 1 \mathrm{H}), 3.34-3.21(\mathrm{~m}$, 2H), $3.10(\mathrm{t}, J=8.2 \mathrm{~Hz}, 2 \mathrm{H}), 2.00-1.88(\mathrm{~m}, 1 \mathrm{H}), 1.88-1.79(\mathrm{~m}, 1 \mathrm{H}), 1.30(\mathrm{~d}, J=6.7 \mathrm{~Hz}, 3 \mathrm{H}) ;{ }^{13} \mathrm{C} \mathrm{NMR}$

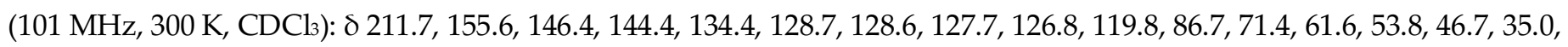
23.8, 14.8; IR (thin film): $\widetilde{v} 3395,3356,2930,1592,1412,1312,1240,1066,706 \mathrm{~cm}^{-1}$; HRMS (ESI): $\mathrm{m} / z$ calculated for $\mathrm{C}_{32} \mathrm{H}_{32} \mathrm{~N}_{2} \mathrm{O}_{2} \mathrm{SNa}^{+}[\mathrm{M}+\mathrm{Na}]^{+}:$531.2077, found: 531.2075; $[\alpha]_{\mathrm{D}}^{26}-36.0$ (c 1.0, $\mathrm{CHCl}_{3}, 96 \%$ ee sample).

Enantiomeric excess was determined to be $96 \%$ ee (procedure $A$ ), by chiral stationary phase HPLC analysis $($ CHIRALPAK IA3 $(\phi 0.46 \mathrm{~cm} \times 25 \mathrm{~cm}), n$-hexane/2-propanol = 95/5, flow rate $1.0 \mathrm{~mL} / \mathrm{min}$, detection at $254 \mathrm{~nm}$, tr $=18.2$ $\min$ (major), $21.5 \mathrm{~min}$ (minor).
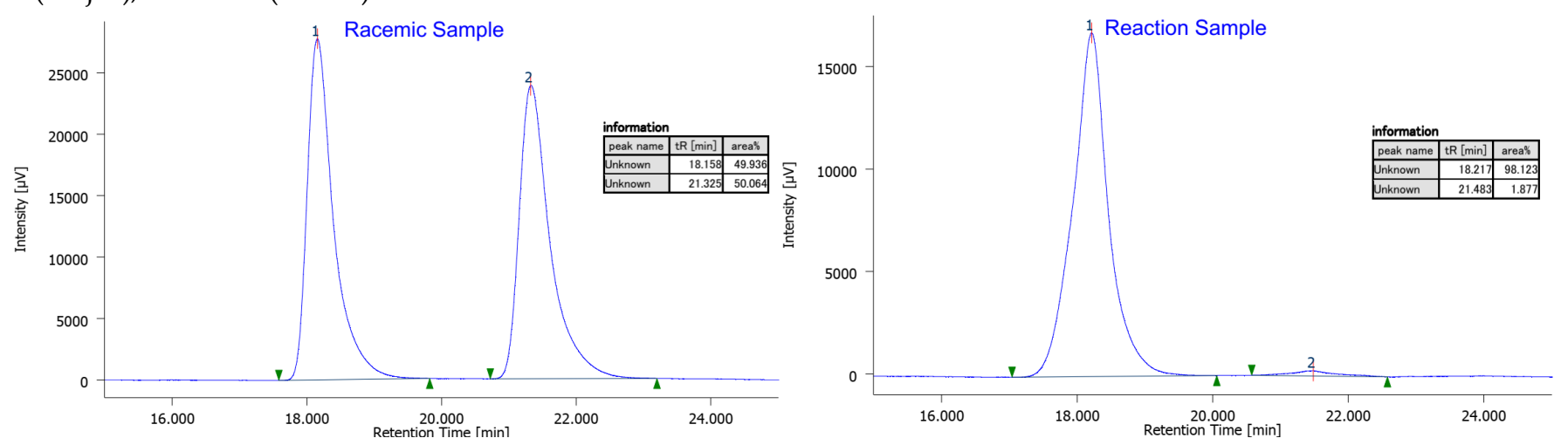

Methyl (6R,7S)-8-(2,3-dihydro-1H-pyrrolo[2,3-b]pyridin-1-yl)-6-hydroxy-7-methyl-8-thioxooctanoate (5f)

Reaction performed according to procedure $A$ gave after purification product 5f $(24.6 \mathrm{mg}$, 70\%, d.r $>20: 1)$. The diastereomeric ratio (d.r.) (ethyl acetate/hexane $=1 / 3$ ) was determined by NMR analysis. 


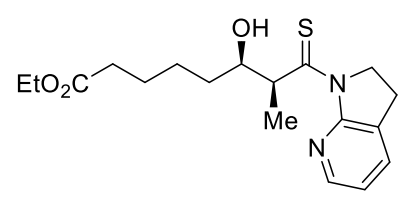
Pale yellow oil; ${ }^{1} \mathrm{H}$ NMR $\left(400 \mathrm{MHz}, 300 \mathrm{~K}, \mathrm{CDCl}_{3}\right): \delta 8.20$ (ddt, J=5.0, 1.6, $\left.1.1 \mathrm{~Hz}, 1 \mathrm{H}\right), 7.61$ (ddd, $J=7.4,2.8,1.1 \mathrm{~Hz}, 1 \mathrm{H}), 7.03(\mathrm{dd}, J=7.4,5.0 \mathrm{~Hz}, 1 \mathrm{H}), 5.28(\mathrm{qd}, J=6.8,1.7 \mathrm{~Hz}, 1 \mathrm{H}), 4.61-$ $4.44(\mathrm{~m}, 2 \mathrm{H}), 4.20(\mathrm{~s}, 1 \mathrm{H}), 4.11(\mathrm{q}, J=7.1 \mathrm{~Hz}, 2 \mathrm{H}), 3.97(\mathrm{ddd}, J=8.5,4.4,1.7 \mathrm{~Hz}, 1 \mathrm{H}), 3.13(\mathrm{t}, J$ $=8.2 \mathrm{~Hz}, 2 \mathrm{H}), 2.31(\mathrm{t}, J=7.6 \mathrm{~Hz}, 2 \mathrm{H}), 1.76-1.60(\mathrm{~m}, 3 \mathrm{H}), 1.60-1.37(\mathrm{~m}, 3 \mathrm{H}), 1.28(\mathrm{~d}, J=6.8 \mathrm{~Hz}$, 3H), $1.24(\mathrm{t}, J=7.1 \mathrm{~Hz}, 3 \mathrm{H}) ;{ }^{13} \mathrm{C}$ NMR $\left(101 \mathrm{MHz}, 300 \mathrm{~K}, \mathrm{CDCl}_{3}\right): \delta$ 212.1, 173.8, 155.6, 146.4, 134.5, 128.8, 119.9, 72.9, 60.2, 53.9, 46.2, 34.4, 34.2, 25.7, 25.0, 23.8, 14.3, 14.2; IR (thin film): $\widetilde{v} 3411,2935,2863,1731,1591,1413,1241,788 \mathrm{~cm}^{-1}$; HRMS (ESI): $m / z$ calculated for $\mathrm{C}_{18} \mathrm{H}_{26} \mathrm{~N}_{2} \mathrm{O}_{3} \mathrm{SNa}^{+}[\mathrm{M}+\mathrm{Na}]^{+}: 373.1556$, found: 373.1556; $[\alpha]_{\mathrm{D}}^{26}-3.1$ ( $1.0, \mathrm{CHCl}_{3}, 97 \%$ ee sample).

Enantiomeric excess was determined to be $97 \%$ ee (procedure A), by chiral stationary phase HPLC analysis (CHIRALPAK IG3 $(\phi 0.46 \mathrm{~cm} \times 25 \mathrm{~cm}), n$-hexane/2-propanol =75/25, flow rate $1.0 \mathrm{~mL} / \mathrm{min}$, detection at $254 \mathrm{~nm}, \mathrm{tr}=19.4$ min (major), $24.1 \mathrm{~min}$ (minor).
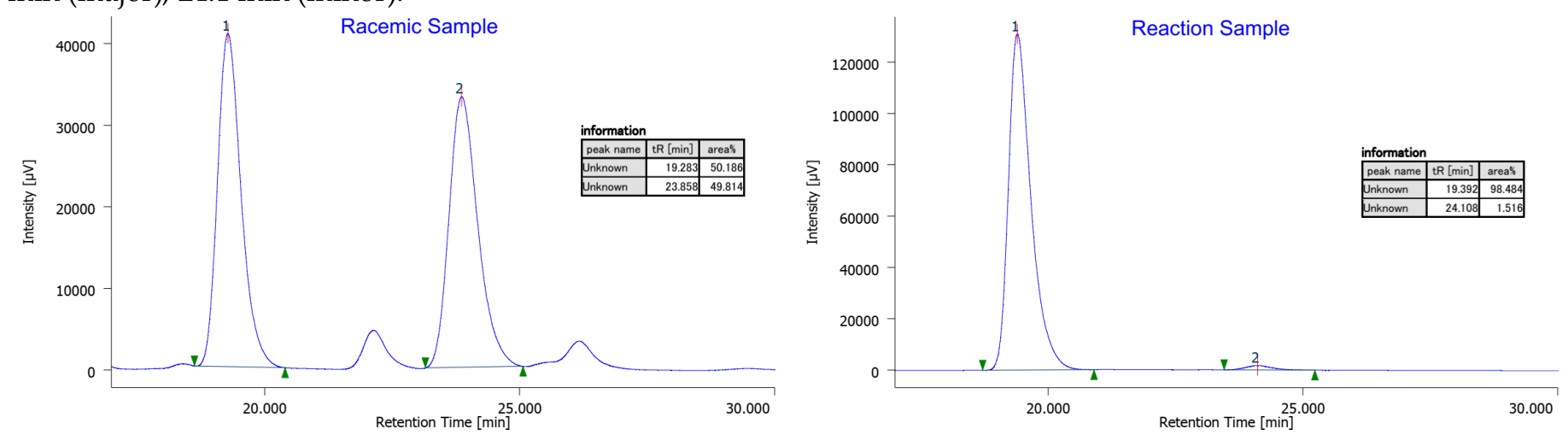

tert-Butyl carbamate (5g)

benzyl((3R,4S)-5-(2,3-dihydro-1H-pyrrolo[2,3-b]pyridin-1-yl)-3-hydroxy-4-methyl-5-thioxopentyl)

Reaction performed according to procedure $A$ gave after purification product $5 \mathrm{~g}(31.0 \mathrm{mg}$, 68\%, d.r. >20:1) (ethyl acetate/hexane $=1 / 1$ ). The diastereomeric ratio (d.r.) was determined by NMR analysis.<smiles>OC(CCNBr)C(=S)N1CCc2cccnc21</smiles>

Pale yellow oil; ${ }^{1} \mathrm{H}$ NMR (400 MHz, $343 \mathrm{~K}, \mathrm{CD}_{3} \mathrm{CN}$ ): $\delta 8.14$ (ddt, J = 5.0, 1.8, $\left.0.9 \mathrm{~Hz}, 1 \mathrm{H}\right), 7.69$ (ddd, $J=7.5,3.0,1.3 \mathrm{~Hz}, 1 \mathrm{H}), 7.34-7.25(\mathrm{~m}, 2 \mathrm{H}), 7.25-7.18(\mathrm{~m}, 3 \mathrm{H}), 7.07(\mathrm{dd}, J=7.5,5.0 \mathrm{~Hz}, 1 \mathrm{H}), 5.34(\mathrm{qd}$, $J=6.6,4.8 \mathrm{~Hz}, 1 \mathrm{H}), 4.50-4.40(\mathrm{~m}, 3 \mathrm{H}), 4.35(\mathrm{~d}, J=15.6 \mathrm{~Hz}, 1 \mathrm{H}), 3.88(\mathrm{dq}, J=4.7,3.6 \mathrm{~Hz}, 1 \mathrm{H}), 3.62(\mathrm{~s}$, $1 \mathrm{H}), 3.40-3.30(\mathrm{~m}, 1 \mathrm{H}), 3.24(\mathrm{ddd}, J=13.9,8.7,4.8 \mathrm{~Hz}, 1 \mathrm{H}), 3.14-3.05(\mathrm{~m}, 2 \mathrm{H}), 1.84-1.71(\mathrm{~m}, 1 \mathrm{H})$, 1.65-1.52 (m, 1H), $1.42(\mathrm{~s}, 9 \mathrm{H}), 1.28$ (d, J = 6.6 Hz, 3H); ${ }^{13} \mathrm{C}$ NMR (101 MHz, $\left.343 \mathrm{~K}, \mathrm{CD}_{3} \mathrm{CN}\right): \delta 212.3,157.1,157.0,147.0$, 140.5, 136.1, 130.9, 129.6, 128.6, 128.1, 121.2, 80.4, 73.7, 55.5, 51.6, 48.0, 45.4, 34.6, 29.0, 24.8, 16.9; IR (thin film): $\widetilde{v} 3420,2974$, 1687, 1591, 1413, 1241, 1165, 966, 786, $700 \mathrm{~cm}^{-1}$; HRMS (ESI): $\mathrm{m} / z$ calculated for $\mathrm{C}_{25} \mathrm{H}_{33} \mathrm{~N}_{3} \mathrm{O}_{3} \mathrm{SNa}^{+}[\mathrm{M}+\mathrm{Na}]^{+}: 478.2135$, found: 478.2135; $[\alpha]_{\mathrm{D}}^{26} 0.5$ ( $c$ 1.0, $\mathrm{CHCl}_{3}, 96 \%$ ee sample).

Enantiomeric excess was determined to be $96 \%$ ee (procedure A), by chiral stationary phase HPLC analysis (CHIRALPAK IG3 $(\phi 0.46 \mathrm{~cm} \times 25 \mathrm{~cm}), n$-hexane $/ 2$-propanol $=75 / 25$, flow rate $1.0 \mathrm{~mL} / \mathrm{min}$, detection at $254 \mathrm{~nm}, \mathrm{t}_{\mathrm{R}}=20.6$ $\min$ (major), $25.0 \mathrm{~min}$ (minor).
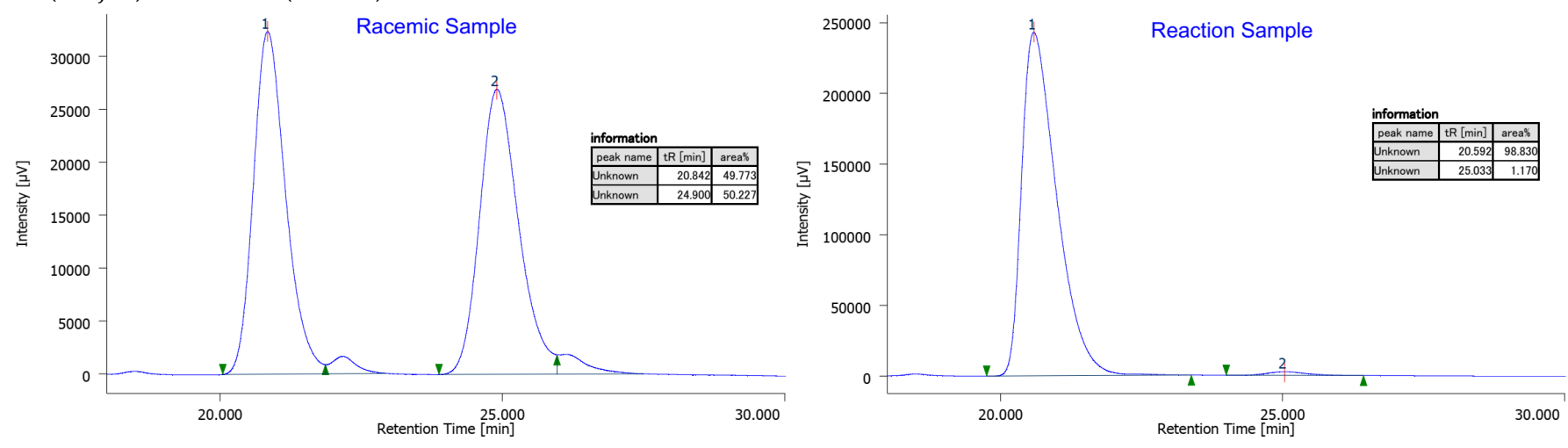
Benzyl 4-((2R,3S)-4-(2,3-dihydro-1H-pyrrolo[2,3-b]pyridin-1-yl)-2-hydroxy-3-methyl-4-thioxobutyl)piperidine-1carboxylate (5h)

Reaction performed according to procedure $A$ gave after purification product $5 \mathbf{h}(30.9 \mathrm{mg}, 68 \%$, d.r. $>20 / 1)$ (ethyl acetate/hexane $=1 / 1$ ). The diastereomeric ratio (d.r.) was determined by NMR analysis.

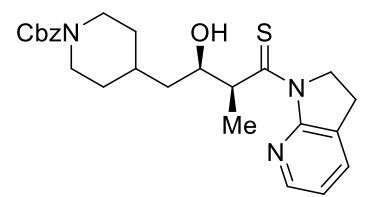

Pale yellow oil; ${ }^{1} \mathrm{H}$ NMR $\left(400 \mathrm{MHz}, 300 \mathrm{~K}, \mathrm{CD}_{3} \mathrm{CN}\right): \delta 8.16$ (ddt, $\left.J=5.0,1.8,0.9 \mathrm{~Hz}, 1 \mathrm{H}\right), 7.70-$ $7.65(\mathrm{~m}, 1 \mathrm{H}), 7.38-7.25(\mathrm{~m}, 5 \mathrm{H}), 7.06(\mathrm{dd}, J=7.5,5.0 \mathrm{~Hz}, 1 \mathrm{H}), 5.29(\mathrm{qd}, J=6.7,4.1 \mathrm{~Hz}, 1 \mathrm{H}), 5.06(\mathrm{~s}$, $2 \mathrm{H}), 4.52-4.32(\mathrm{~m}, 2 \mathrm{H}), 4.07-3.95(\mathrm{~m}, 3 \mathrm{H}), 3.69(\mathrm{~d}, J=3.3 \mathrm{~Hz}, 1 \mathrm{H}), 3.07(\mathrm{t}, J=8.1 \mathrm{~Hz}, 2 \mathrm{H}), 2.75(\mathrm{~s}$, $2 \mathrm{H}), 1.79-1.62(\mathrm{~m}, 2 \mathrm{H}), 1.62-1.54(\mathrm{~m}, 1 \mathrm{H}), 1.42(\mathrm{ddd}, J=13.9,9.4,4.7 \mathrm{~Hz}, 1 \mathrm{H}), 1.36-1.27(\mathrm{~m}, 1 \mathrm{H})$, $1.25(\mathrm{~d}, J=6.7 \mathrm{~Hz}, 3 \mathrm{H}), 1.13-0.91(\mathrm{~m}, 2 \mathrm{H}) ;{ }^{13} \mathrm{C} \mathrm{NMR}\left(101 \mathrm{MHz}, 300 \mathrm{~K}, \mathrm{CD}_{3} \mathrm{CN}\right): \delta 212.0,156.7,155.9,146.8,138.5,135.9$, 130.7, 129.4, 128.8, 128.6, 121.1, 72.1, 67.3, 55.2, 47.7, 45.0, 44.9, 42.2, 33.8, 33.2, 32.2, 24.5, 16.1; IR (thin film): $\widetilde{v} 3393,2929$, 1697, 1590, 1412, 1240, 962, 787, 944, $698 \mathrm{~cm}^{-1}$; HRMS (ESI): $\mathrm{m} / z$ calculated for $\mathrm{C}_{25} \mathrm{H}_{31} \mathrm{~N}_{3} \mathrm{O}_{3} \mathrm{SNa}^{+}[\mathrm{M}+\mathrm{H}]^{+}: 476.1978$, found: 476.1977; $[\alpha]_{\mathrm{D}}^{26}-16.9$ ( $c 1.0, \mathrm{CHCl}_{3}, 98 \%$ ee sample).

Enantiomeric excess was determined to be $98 \%$ ee (procedure A), by chiral stationary phase HPLC analysis (CHIRALPAK IG3 $(\phi 0.46 \mathrm{~cm} \times 25 \mathrm{~cm}$ ), $n$-hexane/2-propanol $=65 / 35$, flow rate $1.0 \mathrm{~mL} / \mathrm{min}$, detection at $254 \mathrm{~nm}$, $\mathrm{t}=28.5$ min (major), 31.9 min (minor).
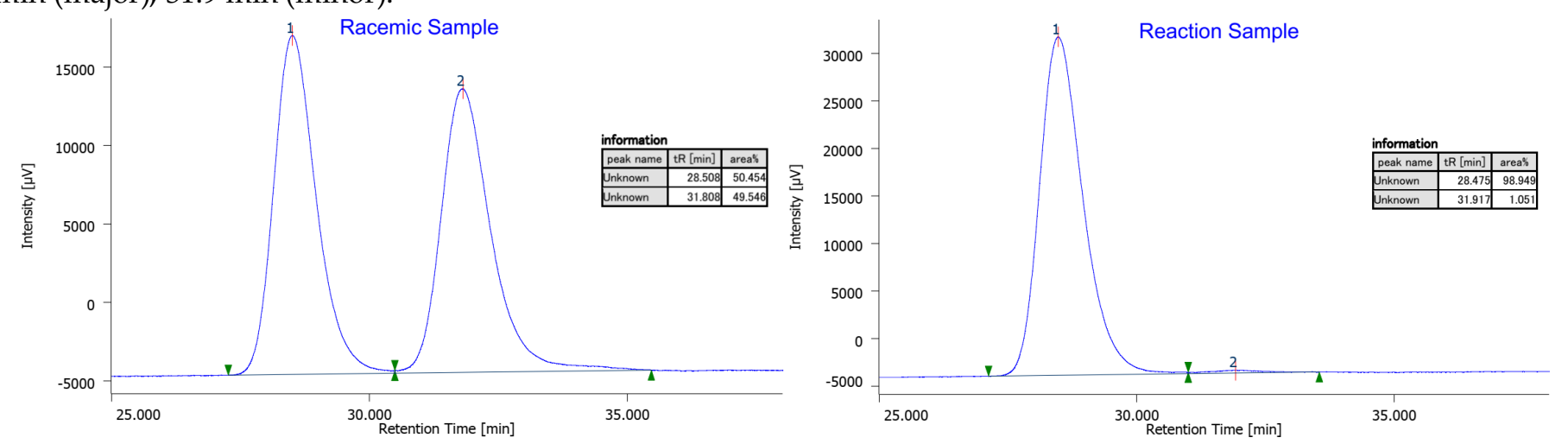

(2S,3R)-1-(2,3-Dihydro-1H-pyrrolo[2,3-b]pyridin-1-yl)-3-hydroxy-2-methyl-8-(triisopropylsilyl)oct-7-yne-1-thione (5i) Reaction performed according to procedure $A$ gave after purification product $5 \mathbf{i}$ (32.1 mg, 72\%, d.r. >20:1) (ethyl acetate/hexane $=1 / 2$ ). The diastereomeric ratio (d.r.) was determined by NMR analysis.

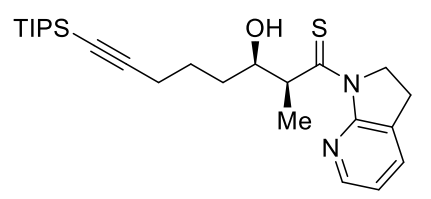

Pale yellow oil ; ${ }^{1} \mathrm{H}$ NMR $\left(400 \mathrm{MHz}, 300 \mathrm{~K}, \mathrm{CDCl}_{3}\right)$ : $\delta$ 8.24- $8.15(\mathrm{~m}, 1 \mathrm{H}), 7.63-7.56(\mathrm{~m}, 1 \mathrm{H})$, $7.02(\mathrm{dd}, J=7.4,5.0 \mathrm{~Hz}, 1 \mathrm{H}), 5.31(\mathrm{qd}, J=6.7,1.5 \mathrm{~Hz}, 1 \mathrm{H}), 4.61-4.42(\mathrm{~m}, 2 \mathrm{H}), 4.28(\mathrm{~s}, 1 \mathrm{H}), 4.06-$ $3.91(\mathrm{~m}, 1 \mathrm{H}), 3.12(\mathrm{t}, J=8.2 \mathrm{~Hz}, 2 \mathrm{H}), 2.41-2.21(\mathrm{~m}, 2 \mathrm{H}), 1.85-1.59(\mathrm{~m}, 4 \mathrm{H}), 1.29(\mathrm{~d}, J=6.8 \mathrm{~Hz}$, $3 \mathrm{H}), 1.11-0.93(\mathrm{~m}, 21 \mathrm{H}) ;{ }^{13} \mathrm{C} \mathrm{NMR}\left(101 \mathrm{MHz}, 300 \mathrm{~K}, \mathrm{CDCl}_{3}\right): \delta 212.2,155.6,146.4,134.5,128.7$, 119.9, 109.2, 80.1, 72.5, 53.8, 46.2, 33.7, 25.6, 23.8, 19.9, 18.6, 14.1, 11.3; IR (thin film): $\widetilde{v} 3402,22941,2864,2169,1591,1414$, 1313, 1241, 883, 786, $676 \mathrm{~cm}^{-1}$; HRMS (ESI): $\mathrm{m} / z$ calculated for $\mathrm{C}_{25} \mathrm{H}_{40} \mathrm{~N}_{2} \mathrm{OSSSiNa}^{+}[\mathrm{M}+\mathrm{Na}]^{+}: 467.2523$, found: 467.2524; $[\alpha]_{\mathrm{D}}^{26} 0.8\left(c 1.0, \mathrm{CHCl}_{3}, 97 \%\right.$ ee sample).

Enantiomeric excess was determined to be $97 \%$ ee (procedure A), by chiral stationary phase HPLC analysis (CHIRALPAK IG3 $\left(\phi 0.46 \mathrm{~cm} \times 25 \mathrm{~cm}\right.$ ), $n$-hexane/2-propanol = 98/2, flow rate $1.0 \mathrm{~mL} / \mathrm{min}$, detection at $254 \mathrm{~nm}$, $\mathrm{tR}_{\mathrm{R}}=17.6$ $\min$ (major), $20.0 \mathrm{~min}$ (minor). 

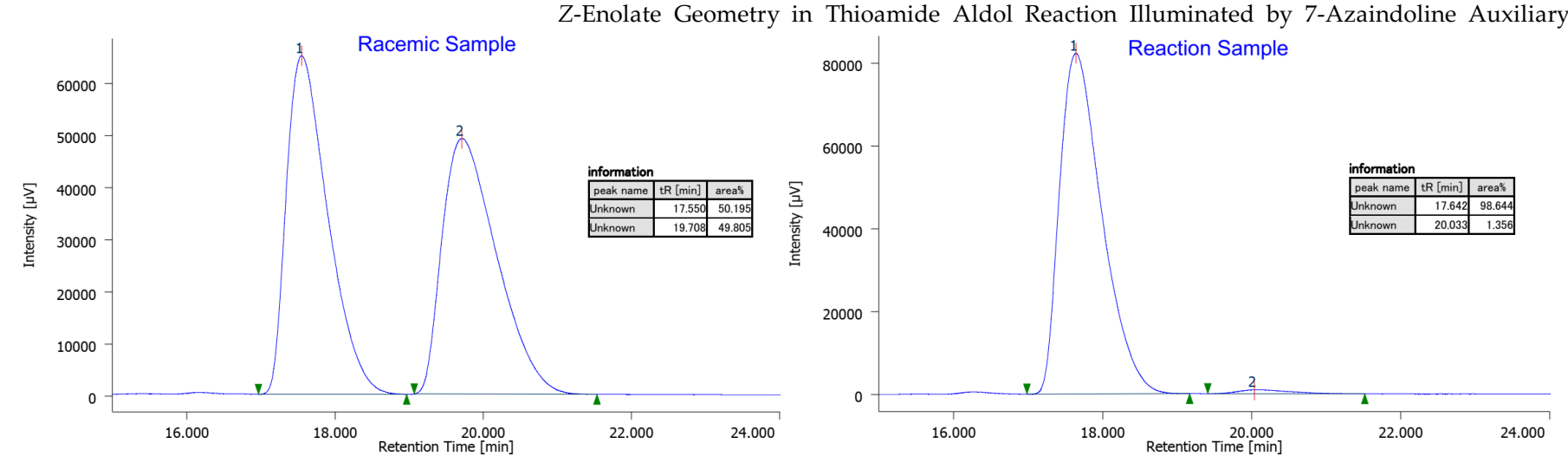

(2S,3R)-1-(2,3-Dihydro-1H-pyrrolo[2,3-b]pyridin-1-yl)-3-hydroxy-2-methyl-5-(tetrahydro-2H-thiopyran-4-yl)pentane1-thione (5j)

Reaction performed according to procedure $A$ gave after purification product 5j (15.8 mg, 45\%, d.r. 9:1) (ethyl acetate/hexane $=2 / 3$ ). The diastereomeric ratio (d.r.) was determined by NMR analysis.

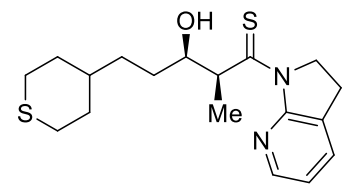

Pale yellow oil ; ${ }^{1} \mathrm{H}$ NMR (400 MHz, $\left.300 \mathrm{~K}, \mathrm{CDCl}_{3}\right)$ : $\delta$ 8.24-8.16 (m, 1H), 7.65-7.57 (m, 1H), 7.04 $(\mathrm{dd}, J=7.4,5.0 \mathrm{~Hz}, 1 \mathrm{H}), 5.29(\mathrm{qd}, J=6.7,1.6 \mathrm{~Hz}, 1 \mathrm{H}), 4.61-4.45(\mathrm{~m}, 2 \mathrm{H}), 4.29(\mathrm{~s}, 1 \mathrm{H}), 3.97-3.87(\mathrm{~m}$, $1 \mathrm{H}), 3.17-3.08(\mathrm{~m}, 2 \mathrm{H}), 2.72-2.51(\mathrm{~m}, 4 \mathrm{H}), 2.09-1.96(\mathrm{~m}, 2 \mathrm{H}), 1.68-1.55(\mathrm{~m}, 1 \mathrm{H}), 1.55-1.42(\mathrm{~m}, 2 \mathrm{H})$, $1.42-1.30(\mathrm{~m}, 4 \mathrm{H}), 1.28(\mathrm{~d}, J=6.8 \mathrm{~Hz}, 3 \mathrm{H}) ;{ }^{13} \mathrm{C} \mathrm{NMR}\left(101 \mathrm{MHz}, 300 \mathrm{~K}, \mathrm{CDCl}_{3}\right): \delta 212.1,155.6,146.3$, 134.6, 128.8, 119.9, 73.3, 53.9, 46.2, 37.1, 34.2, 34.2, 33.7, 31.3, 28.8, 23.8, 14.2; IR (thin film): $\widetilde{v} 3405,2917,1591,1411,1239$, 962, 916, $787 \mathrm{~cm}^{-1}$; HRMS (ESI): $m / z$ calculated for $\mathrm{C}_{18} \mathrm{H}_{26} \mathrm{~N}_{2} \mathrm{OSS}_{2} \mathrm{Na}^{+}[\mathrm{M}+\mathrm{Na}]^{+}: 373.1379$, found: $373.1377 ;[\alpha]_{\mathrm{D}}^{26}-6.4(c 1.0$, $\mathrm{CHCl}_{3}, 96 \%$ ee sample).

Enantiomeric excess was determined to be $96 \%$ ee (procedure $A$ ), by chiral stationary phase HPLC analysis $($ CHIRALPAK IC3 $(\phi 0.46 \mathrm{~cm} \times 25 \mathrm{~cm}), n$-hexane/2-propanol = 85/15, flow rate $1.0 \mathrm{~mL} / \mathrm{min}$, detection at $254 \mathrm{~nm}$, tr $=36.1$ min (minor), 64.7 min (major).
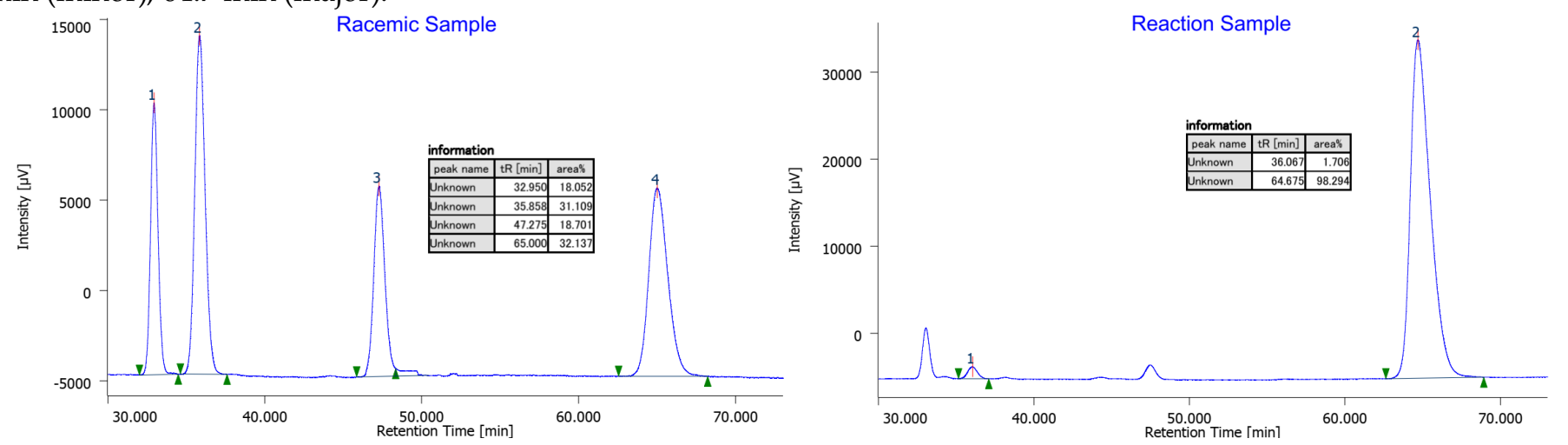

(2S,3R)-1-(2,3-Dihydro-1H-pyrrolo[2,3-b]pyridin-1-yl)-3-hydroxy-2-ethyl-5-phenylpentane-1-thione (5k)

Reaction performed according to procedure $A$ gave after purification product 5k (23.9 mg, 70\%, d.r. 20/1) (ethyl acetate/hexane $=1 / 3$ ). The diastereomeric ratio (d.r.) was determined by NMR analysis.

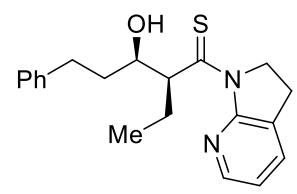

Pale yellow oil; ${ }^{1} \mathrm{H}$ NMR (400 MHz, $\left.300 \mathrm{~K}, \mathrm{CDCl}_{3}\right): \delta 8.18-8.12(\mathrm{~m}, 1 \mathrm{H}), 7.59$ (ddd, J = 7.4, 2.8, $1.2 \mathrm{~Hz}$, $1 \mathrm{H}), 7.31-7.10(\mathrm{~m}, 5 \mathrm{H}), 7.01(\mathrm{dd}, J=7.4,5.0 \mathrm{~Hz}, 1 \mathrm{H}), 5.58(\mathrm{ddd}, J=10.2,3.8,2.6 \mathrm{~Hz}, 1 \mathrm{H}), 4.66-4.43(\mathrm{~m}$, 2H), $4.19(\mathrm{~s}, 1 \mathrm{H}), 4.00-3.87(\mathrm{~m}, 1 \mathrm{H}), 3.22-3.01(\mathrm{~m}, 2 \mathrm{H}), 2.90(\mathrm{ddd}, J=13.8,10.3,5.3 \mathrm{~Hz}, 1 \mathrm{H}), 2.70(\mathrm{ddd}$, $J=13.8,10.0,6.5 \mathrm{~Hz}, 1 \mathrm{H}), 2.19-1.95(\mathrm{~m}, 2 \mathrm{H}), 1.94-1.78(\mathrm{~m}, 2 \mathrm{H}), 0.83(\mathrm{t}, J=7.4 \mathrm{~Hz}, 3 \mathrm{H}) ;{ }^{13} \mathrm{C}$ NMR $(101$ $\left.\mathrm{MHz}, 300 \mathrm{~K}, \mathrm{CDCl}_{3}\right): \delta 210.7,155.9,145.8,142.6,134.7,128.9,128.5,128.2,125.6,120.0,73.4,54.2,52.6,36.4,32.6,23.9,23.8$, 11.9; IR (thin film): $\widetilde{v} 3390,3024,2958,2871,1592,1412,1238,981,787,700 \mathrm{~cm}^{-1}$; HRMS (ESI): $m / z$ calculated for $\mathrm{C}_{20} \mathrm{H}_{25} \mathrm{~N}_{2} \mathrm{OS}^{+}[\mathrm{M}+\mathrm{H}]^{+}:$341.1682, found: 341.1684; $[\alpha]_{\mathrm{D}}^{26}-5.7$ (c 1.0, $\mathrm{CHCl}_{3}, 92 \%$ ee sample).

Enantiomeric excess was determined to be $92 \%$ ee (procedure $A$ ), by chiral stationary phase HPLC analysis 

(CHIRALPAK IG3 $(\phi 0.46 \mathrm{~cm} \times 25 \mathrm{~cm}), n$-hexane/2-propanol =90/10, flow rate $1.0 \mathrm{~mL} / \mathrm{min}$, detection at $254 \mathrm{~nm}$, tR $=27.3$ min (minor), 36.5 min (major).
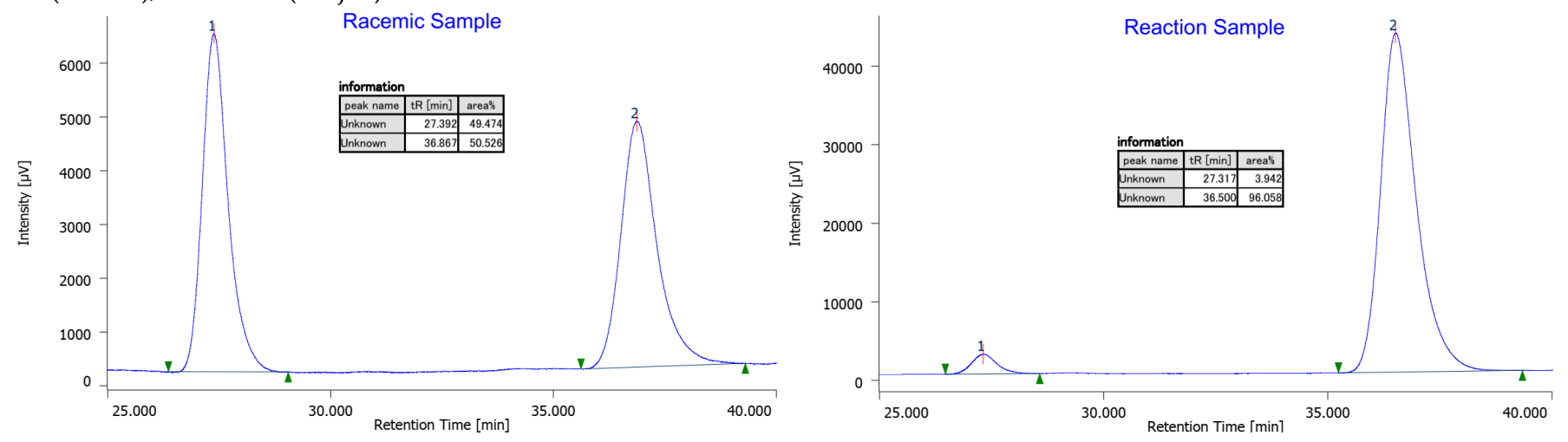

(2S,3R)-2-Benzyl-1-(2,3-dihydro-1H-pyrrolo[2,3-b]pyridin-1-yl)-3-hydroxy-5-phenylpentane-1-thione (51)

Reaction performed according to procedure $B$ gave after purification product 51 (25.0 mg, 62\%, d.r. 20/1) (ethyl acetate/hexane $=1 / 2$ ). The diastereomeric ratio (d.r.) was determined by NMR analysis.

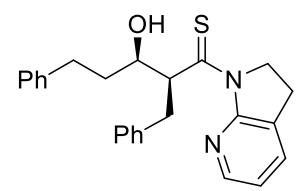

Pale yellow oil; ${ }^{1} \mathrm{H}$ NMR (400 MHz, 300 K, CDCl 3$): \delta 8.14-8.07$ (m, 1H), 7.42 (ddd, J = 7.4, 2.6, 1.2 Hz, $1 \mathrm{H}), 7.31-7.22(\mathrm{~m}, 4 \mathrm{H}), 7.21-7.13(\mathrm{~m}, 1 \mathrm{H}), 7.11-7.06(\mathrm{~m}, 2 \mathrm{H}), 7.03-6.95(\mathrm{~m}, 3 \mathrm{H}), 6.93(\mathrm{dd}, J=7.4,5.0$ $\mathrm{Hz}, 1 \mathrm{H}), 6.09$ (ddd, $J=10.1,5.0,2.2 \mathrm{~Hz}, 1 \mathrm{H}), 4.41-4.31(\mathrm{~m}, 2 \mathrm{H}), 4.23(\mathrm{ddd}, J=13.2,9.9,8.0 \mathrm{~Hz}, 1 \mathrm{H})$, 4.16-4.09 (m, 1H), $3.24(\mathrm{dd}, J=13.3,10.2 \mathrm{~Hz}, 1 \mathrm{H}), 3.15(\mathrm{dd}, J=13.3,5.0 \mathrm{~Hz}, 1 \mathrm{H}), 2.95(\mathrm{ddd}, J=13.8$, $10.3,5.4 \mathrm{~Hz}, 1 \mathrm{H}), 2.89-2.65(\mathrm{~m}, 3 \mathrm{H}), 2.13-2.00(\mathrm{~m}, 1 \mathrm{H}), 2.00-1.87(\mathrm{~m}, 1 \mathrm{H}) ;{ }^{13} \mathrm{C} \mathrm{NMR}(101 \mathrm{MHz}, 300 \mathrm{~K}, \mathrm{CDCl}): \delta 209.6,155.5$, $145.5,142.6,139.6,134.3,129.2,128.7,128.6,128.3,127.5,125.7,125.7,119.9,73.0,54.1,52.0,37.1,36.6,32.5,23.8$; IR (thin film): $\widetilde{v} 3393,3025,2926,1592,1495,1413,1239,1128,1030,787,746,699 \mathrm{~cm}^{-1}$; HRMS (ESI): $m / z$ calculated for $\mathrm{C}_{25} \mathrm{H}_{27} \mathrm{~N}_{2} \mathrm{OS}^{+}$ $[\mathrm{M}+\mathrm{H}]^{+}:$403.1839, found: 403.1841; $[\alpha]_{\mathrm{D}}^{26}-60.1$ ( c 1.0, $\mathrm{CHCl}_{3}, 87 \%$ ee sample).

Enantiomeric excess was determined to be $87 \%$ ee (procedure $B$ ), by chiral stationary phase HPLC analysis $($ CHIRALPAK IG3 $(\phi 0.46 \mathrm{~cm} \times 25 \mathrm{~cm}), n$-hexane/2-propanol = 85/15, flow rate $1.0 \mathrm{~mL} / \mathrm{min}$, detection at $254 \mathrm{~nm}$, tr $=31.1$ min (minor), 39.7 min (major).
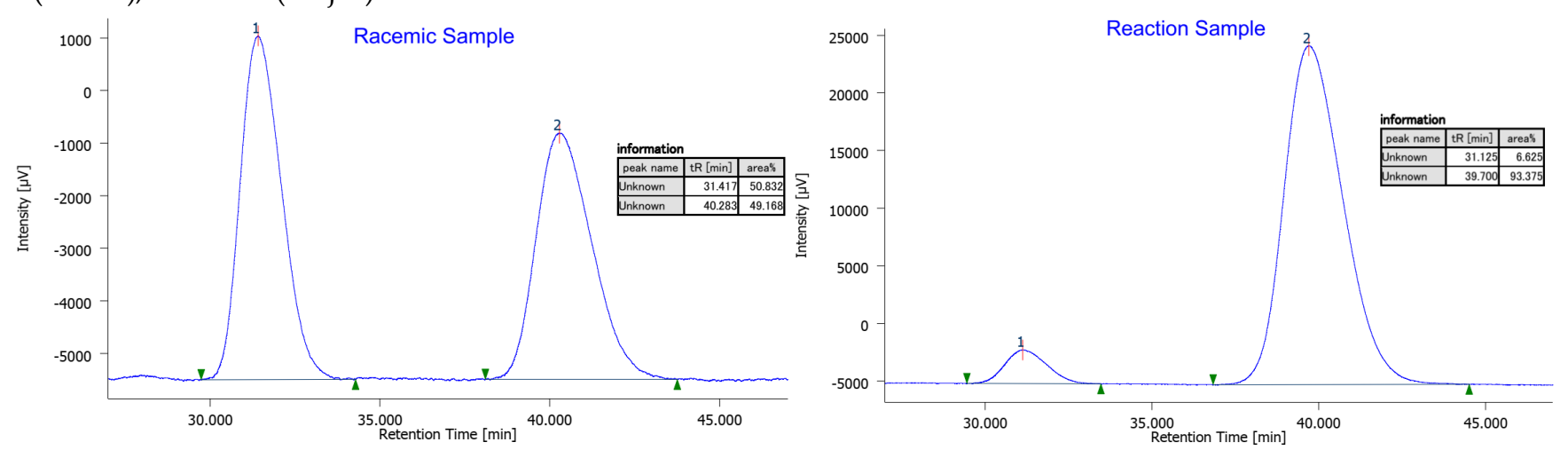

(2S,3R)-2-((Benzyloxy)methyl)-1-(2,3-dihydro-1H-pyrrolo[2,3-b]pyridin-1-yl)-3-hydroxy-5-phenylpentane-1-thione (5m)

Reaction performed according to gram-scale procedure gave after purification product 5m (21.2 mg, 65\%, d.r. 15/1) (ethyl acetate/hexane $=1 / 1$ ). The diastereomeric ratio (d.r.) was determined by NMR analysis.

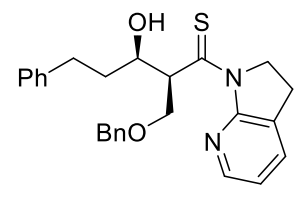

Pale yellow oil; ${ }^{1} \mathrm{H}$ NMR (400 MHz, $\left.300 \mathrm{~K}, \mathrm{CDCl}_{3}\right): \delta 8.17(\mathrm{ddt}, J=5.0,1.7,1.1 \mathrm{~Hz}, 1 \mathrm{H}), 7.56(\mathrm{dq}, J=$ $2.9,1.1 \mathrm{~Hz}, 1 \mathrm{H}), 7.29-7.20(\mathrm{~m}, 7 \mathrm{H}), 7.20-7.16(\mathrm{~m}, 2 \mathrm{H}), 7.16-7.11(\mathrm{~m}, 1 \mathrm{H}), 7.00(\mathrm{dd}, J=7.4,5.0 \mathrm{~Hz}, 1 \mathrm{H})$, $5.95(\mathrm{td}, J=6.6,3.8 \mathrm{~Hz}, 1 \mathrm{H}), 4.57(\mathrm{ddd}, J=13.5,8.6,6.9 \mathrm{~Hz}, 1 \mathrm{H}), 4.52(\mathrm{~s}, 2 \mathrm{H}), 4.43(\mathrm{ddd}, J=13.5,9.1$, $7.9 \mathrm{~Hz}, 1 \mathrm{H}), 4.32(\mathrm{~s}, 1 \mathrm{H}), 4.14(\mathrm{dt}, J=8.6,3.9 \mathrm{~Hz}, 1 \mathrm{H}), 4.11-4.04(\mathrm{~m}, 2 \mathrm{H}), 3.09-3.02(\mathrm{~m}, 2 \mathrm{H}), 2.98-2.84$ (m, 1H), 2.76-2.62 (m, 1H), 2.07-1.89 (m, 2H); $\left.{ }^{13} \mathrm{C} \mathrm{NMR} \mathrm{(101} \mathrm{MHz,} 300 \mathrm{~K}, \mathrm{CDCl}_{3}\right): \delta 206.3,155.8,146.0,142.5,138.3,134.6$, $128.8,128.5,128.2,128.2,127.5,127.4,125.6,120.0,73.7,73.3,72.7,54.1,51.8,36.4,32.4,23.9$; IR (thin film): $\widetilde{v} 3408,3025$, 

2921, 2860, 1592, 1412, 1239, 1099, 787, 741, $699 \mathrm{~cm}^{-1}$; HRMS (ESI): m/z calculated for $\mathrm{C}_{26} \mathrm{H}_{29} \mathrm{~N}_{2} \mathrm{O}_{2} \mathrm{~S}^{+}\left[\mathrm{M}^{+} \mathrm{H}\right]^{+}: 433.1944$, found: $433.1948 ;[\alpha]_{\mathrm{D}}^{26}-14.3$ (c 1.0, $\mathrm{CHCl}_{3}, 91 \%$ ee sample).

Enantiomeric excess was determined to be $91 \%$ ee (procedure B ), by chiral stationary phase HPLC analysis $($ CHIRALPAK IG3 $(\phi 0.46 \mathrm{~cm} \times 25 \mathrm{~cm}), n$-hexane/2-propanol = 75/25, flow rate $1.0 \mathrm{~mL} / \mathrm{min}$, detection at $254 \mathrm{~nm}$, tr $=20.3$ min (major), $25.2 \mathrm{~min}$ (minor).
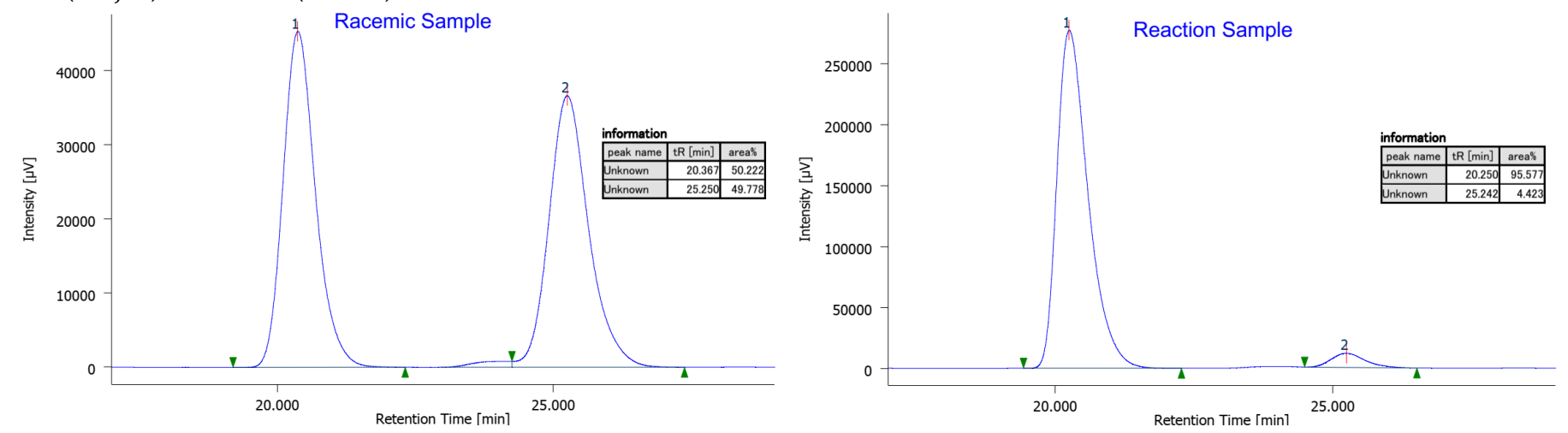

Ethyl (4S,5R)-4-(2,3-dihydro-1H-pyrrolo[2,3-b]pyridine-1-carbonothioyl)-5-hydroxy-7-phenylheptanoate (5n)

Reaction performed according to procedure $A$ gave after purification product 5 n $(28.1 \mathrm{mg}$, 68\%, d.r. 9/1) (ethyl acetate/hexane $=1 / 1$ ). The diastereomeric ratio (d.r.) was determined by NMR analysis.

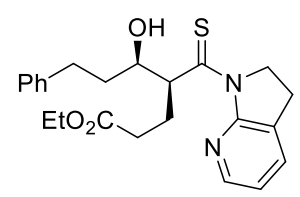

Pale yellow oil; ${ }^{1} \mathrm{H}$ NMR (400 MHz, $300 \mathrm{~K}, \mathrm{CDCl}_{3}$ ): $\delta 8.12$ (ddt, $\left.J=4.9,1.7,1.1 \mathrm{~Hz}, 1 \mathrm{H}\right), 7.60$ (ddd, $J$ $=7.5,2.9,1.1 \mathrm{~Hz}, 1 \mathrm{H}), 7.28-7.18(\mathrm{~m}, 4 \mathrm{H}), 7.18-7.11(\mathrm{~m}, 1 \mathrm{H}), 7.02(\mathrm{dd}, J=7.4,5.0 \mathrm{~Hz}, 1 \mathrm{H}), 5.66(\mathrm{dt}, J=$ 5.9, 3.2 Hz, 1H), 4.63-4.46 (m, 2H), 4.16-3.88 (m, 4H), 3.21-3.03 (m, 2H), $2.88(\mathrm{ddd}, J=14.0,10.2,5.3$ $\mathrm{Hz}, 1 \mathrm{H}), 2.71(\mathrm{ddd}, J=14.0,9.9,6.5 \mathrm{~Hz}, 1 \mathrm{H}), 2.50-2.14(\mathrm{~m}, 4 \mathrm{H}), 2.08-1.96(\mathrm{~m}, 1 \mathrm{H}), 1.94-1.82(\mathrm{~m}, 1 \mathrm{H})$, $1.14(\mathrm{t}, J=7.1 \mathrm{~Hz}, 3 \mathrm{H}) ;{ }^{13} \mathrm{C} \mathrm{NMR}\left(101 \mathrm{MHz}, 300 \mathrm{~K}, \mathrm{CDCl}_{3}\right)$ : $\delta$ 209.2, 173.5, 155.7, 145.9, 142.5, 134.8, 129.0, 128.5, 128.3, 125.6, 120.1, 73.3, 60.2, 54.3, 50.2, 36.3, 32.4, 32.1, 25.8, 23.9, 14.2; IR (thin film): $\widetilde{v} 3931,1732,1592,1413,1351,1248,1038,789$, 747, $700 \mathrm{~cm}^{-1}$; HRMS (ESI): $m / z$ calculated for $\mathrm{C}_{23} \mathrm{H}_{29} \mathrm{~N}_{2} \mathrm{O}_{3} \mathrm{~S}^{+}[\mathrm{M}+\mathrm{H}]^{+}: 413.1893$, found: 413.1898; $[\alpha]_{\mathrm{D}}^{26} 71.6(c$ 1.0, $\mathrm{CHCl}$, $94 \%$ ee sample).

Enantiomeric excess was determined to be $94 \%$ ee (procedure B ), by chiral stationary phase HPLC analysis $\left(\right.$ CHIRALPAK IG3 $(\phi 0.46 \mathrm{~cm} \times 25 \mathrm{~cm}), n$-hexane/ethanol $=65 / 35$, flow rate $1.0 \mathrm{~mL} / \mathrm{min}$, detection at $254 \mathrm{~nm}$, $\mathrm{t}_{\mathrm{R}}=18.1 \mathrm{~min}$ (minor), $12.1 \mathrm{~min}$ (major).
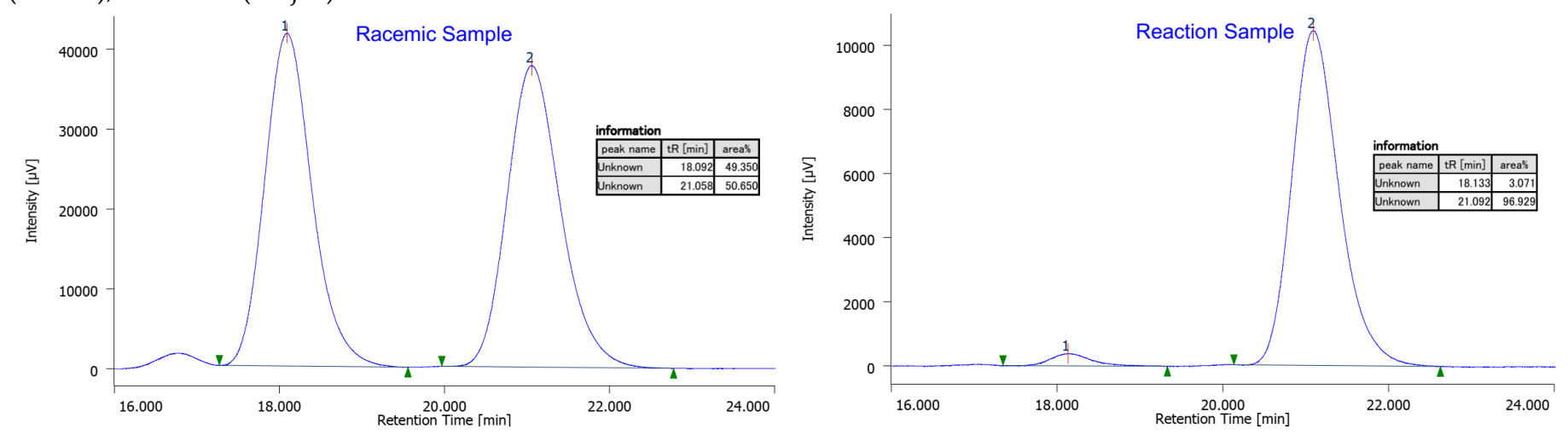

(2S,3R)-1-(2,3-Dihydro-1H-pyrrolo[2,3-b]pyridin-1-yl)-3-hydroxy-2-methyl-5-phenyl-2-(2,2,2-trifluoroethyl)pentane1-thione (5o)

Reaction performed according to procedure $A$ gave after purification product 50 (24.1 mg, 61\%, d.r. 10/1) (ethyl acetate/hexane $=1 / 2$ ). The diastereomeric ratio (d.r.) was determined by NMR analysis. 


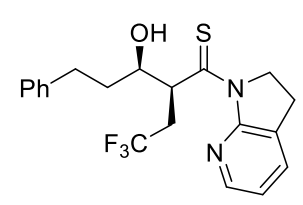
Pale yellow oil; ${ }^{1} \mathrm{H}$ NMR $\left(400 \mathrm{MHz}, 300 \mathrm{~K}, \mathrm{CDCl}_{3}\right): \delta 8.18-8.14(\mathrm{~m}, 1 \mathrm{H}), 7.61(\mathrm{dq}, J=7.5,1.4 \mathrm{~Hz}, 1 \mathrm{H})$, 7.29-7.23 (m, 2H), 7.23-7.19 (m, 2H), 7.19-7.13 (m, 1H), 7.04 (dd, J = 7.5, 5.0 Hz, 1H), $6.18(\mathrm{~d}, J=10.3$ $\mathrm{Hz}, 1 \mathrm{H}), 4.61(\mathrm{ddd}, J=13.8,9.9,5.1 \mathrm{~Hz}, 1 \mathrm{H}), 4.46(\mathrm{ddd}, J=13.6,10.0,7.4 \mathrm{~Hz}, 1 \mathrm{H}), 3.92-3.85(\mathrm{~m}, 1 \mathrm{H})$, $3.79(\mathrm{~s}, 1 \mathrm{H}), 3.30(\mathrm{dqd}, J=15.3,11.3,10.6 \mathrm{~Hz}, 1 \mathrm{H}), 3.21-3.03(\mathrm{~m}, 2 \mathrm{H}), 2.90(\mathrm{ddd}, J=14.3,9.9,5.6 \mathrm{~Hz}$, 1H), 2.75 (ddd, $J=13.9,9.6,6.8 \mathrm{~Hz}, 1 \mathrm{H}), 2.59$ (dqd, $J=15.3,11.3,1.7 \mathrm{~Hz}, 1 \mathrm{H}), 2.05-1.85$ (m, 2H); ${ }^{13} \mathrm{C}$ NMR $(101 \mathrm{MHz}, 300$ $\left.\mathrm{K}, \mathrm{CDCl}_{3}\right): \delta$ 207.0, 155.6, 146.0, 142.0, 134.9, 128.8, 128.5, 128.4, 127.3 (q, $\left.J=277.1 \mathrm{~Hz}\right), 125.8,120.4,72.7,54.5,44.8$ (q, $J=$ $2.1 \mathrm{~Hz}), 36.2,35.0$ (q, J = 28.0 Hz), 32.1, 23.9; $\left.{ }^{19} \mathrm{~F} \mathrm{NMR} \mathrm{(376} \mathrm{MHz,} 300 \mathrm{~K}, \mathrm{CDCl}_{3}\right)$ : $\delta-64.5(\mathrm{t}, J=11.3 \mathrm{~Hz}, 3 \mathrm{~F})$; IR (thin film): $\widetilde{v} 3394,2951,1593,1414,1372,1238,1136,788,747,700 \mathrm{~cm}^{-1}$; HRMS (ESI): $\mathrm{m} / z$ calculated for $\mathrm{C}_{20} \mathrm{H}_{22} \mathrm{~N}_{2} \mathrm{OF}_{3} \mathrm{~S}^{+}[\mathrm{M}+\mathrm{H}]^{+}$: 395.1399, found: 395.1404; $[\alpha]_{\mathrm{D}}^{26} 15.0\left(c 1.0, \mathrm{CHCl}_{3}, 88 \%\right.$ ee sample).

Enantiomeric excess was determined to be $88 \%$ ee (procedure B), by chiral stationary phase HPLC analysis (CHIRALPAK IG3 $(\phi 0.46 \mathrm{~cm} \times 25 \mathrm{~cm}), n$-hexane/2-propanol $=90 / 10$, flow rate $1.0 \mathrm{~mL} / \mathrm{min}$, detection at $254 \mathrm{~nm}, \mathrm{tr}=12.8$ $\min$ (major), $14.9 \mathrm{~min}$ (minor).
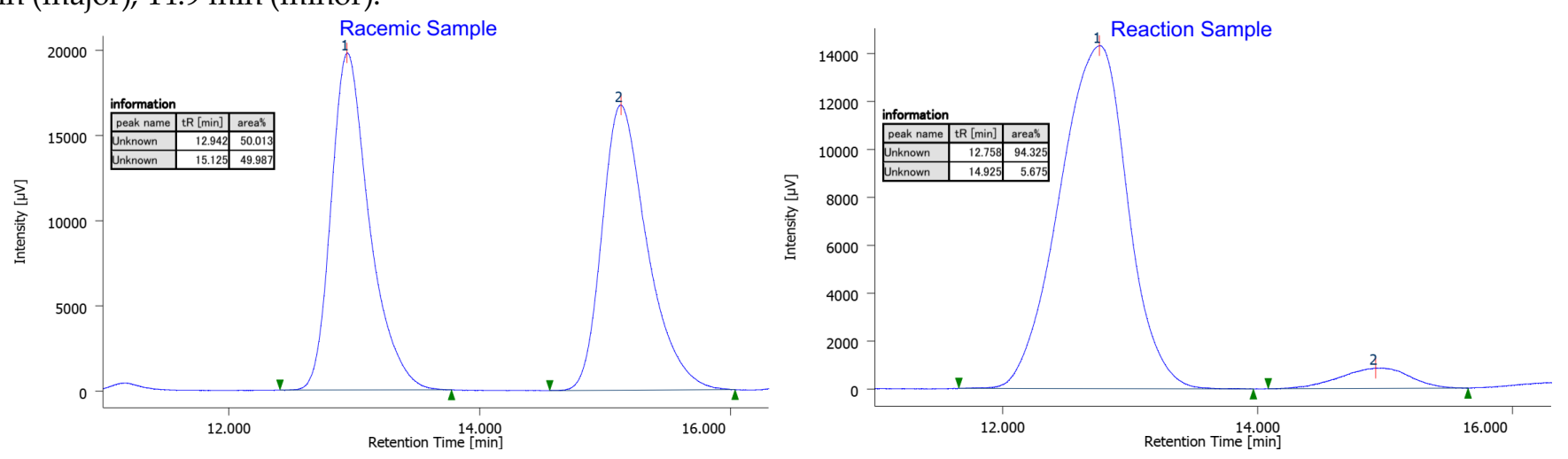

(2S,3R,5S)-1-(2,3-dihydro-1H-pyrrolo[2,3-b]pyridin-1-yl)-3-hydroxy-2,5,9-trimethyldec-8-ene-1-thione (5p)

Reaction performed according to procedure $A$ conducted with $(S, S)$-Ph-BPE gave after purification product 5 p $(26.0 \mathrm{mg}$, $75 \%$, d.r. 15/1) (ethyl acetate/hexane =1/3). The diastereomeric ratio (d.r.) was determined by NMR analysis.

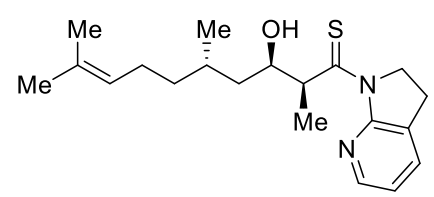

Pale yellow oil; ${ }^{1} \mathrm{H}$ NMR (400 MHz, $\left.300 \mathrm{~K}, \mathrm{CDCl}_{3}\right)$ : $\delta$ 8.23-8.18 (m, 1H), 7.60 (ddd, J = 7.4, $2.8,1.2 \mathrm{~Hz}, 1 \mathrm{H}), 7.02(\mathrm{dd}, J=7.4,5.0 \mathrm{~Hz}, 1 \mathrm{H}), 5.20(\mathrm{qd}, J=6.7,1.7 \mathrm{~Hz}, 1 \mathrm{H}), 5.12-5.06(\mathrm{~m}, 1 \mathrm{H})$, 4.60-4.46 (m, 2H), $4.17(\mathrm{~s}, 1 \mathrm{H}), 4.14-4.06(\mathrm{~m}, 1 \mathrm{H}), 3.12(\mathrm{t}, J=8.2 \mathrm{~Hz}, 2 \mathrm{H}), 2.10-1.88(\mathrm{~m}, 2 \mathrm{H})$, 1.80-1.66 (m, 2H), $1.65(\mathrm{~d}, J=1.0 \mathrm{~Hz}, 3 \mathrm{H}), 1.58(\mathrm{~s}, 3 \mathrm{H}), 1.40-1.32(\mathrm{~m}, 1 \mathrm{H}), 1.30(\mathrm{~d}, J=6.7 \mathrm{~Hz}$, 3H), 1.24-1.11 (m, 2H), 0.92 (d, J = 6.6 Hz, 3H); $\left.{ }^{13} \mathrm{C} \mathrm{NMR} \mathrm{(101} \mathrm{MHz,} 300 \mathrm{~K}, \mathrm{CDCl}_{3}\right): \delta$ 212.4, 155.6, 146.4, 134.5, 130.9, 128.7, 125.0, 119.9, 70.8, 53.9, 46.9, 42.1, 38.1, 29.0, 25.7, 25.6, 23.8, 19.1, 17.6, 14.3; IR (thin film): $\widetilde{v} 3397,3925,1591,1413,1311$, 1240, 913, 786, $744 \mathrm{~cm}^{-1}$; HRMS (ESI): $m / z$ calculated for $\mathrm{C}_{20} \mathrm{H}_{30} \mathrm{~N}_{2} \mathrm{OSNa}^{+}[\mathrm{M}+\mathrm{Na}]^{+}: 369.1971$, found: $369.1974 ;[\alpha]_{\mathrm{D}}^{26}-7.9(c$ $\left.1.0, \mathrm{CHCl}_{3}\right)$.

(2R,3S,5S)-1-(2,3-dihydro-1H-pyrrolo[2,3-b]pyridin-1-yl)-3-hydroxy-2,5,9-trimethyldec-8-ene-1-thione (5q)

Reaction performed according to procedure $A$ with $(R, R)$-Ph-BPE (ent-L5) gave after purification product $\mathbf{5 q}(15.3 \mathbf{m g}$, $44 \%$, d.r. 15/1) (ethyl acetate/hexane =1/3). The diastereomeric ratio (d.r.) was determined by NMR analysis.

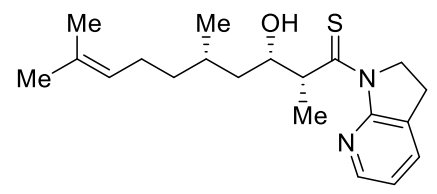

Pale yellow oil; ${ }^{1} \mathrm{H}$ NMR (400 MHz, $\left.300 \mathrm{~K}, \mathrm{CDCl}_{3}\right)$ : $\delta$ 8.21-8.16 (m, 1H), 7.60 (ddd, J = 7.4, $2.9,1.2 \mathrm{~Hz}, 1 \mathrm{H}), 7.02(\mathrm{dd}, J=7.4,5.0 \mathrm{~Hz}, 1 \mathrm{H}), 5.28(\mathrm{qd}, J=6.7,1.5 \mathrm{~Hz}, 1 \mathrm{H}), 5.14-5.06(\mathrm{~m}, 1 \mathrm{H})$, 4.61-4.46 (m, 2H), $4.22(\mathrm{~s}, 1 \mathrm{H}), 4.13-4.05(\mathrm{~m}, 1 \mathrm{H}), 3.13(\mathrm{t}, J=8.2 \mathrm{~Hz}, 2 \mathrm{H}), 2.11-1.89(\mathrm{~m}, 2 \mathrm{H})$, $1.78-1.61(\mathrm{~m}, 4 \mathrm{H}), 1.59(\mathrm{~s}, 3 \mathrm{H}), 1.55-1.39(\mathrm{~m}, 3 \mathrm{H}), 1.29(\mathrm{~d}, J=6.8 \mathrm{~Hz}, 3 \mathrm{H}), 1.14(\mathrm{dddd}, J=13.5$, 9.8, 8.0, $5.8 \mathrm{~Hz}, 1 \mathrm{H}), 0.92$ (d, J = 6.7 Hz, 3H); ${ }^{13} \mathrm{C} \mathrm{NMR}\left(101 \mathrm{MHz}, 300 \mathrm{~K}, \mathrm{CDCl}_{3}\right): \delta$ 212.5, 155.7, 146.3, 134.5, 130.9, 128.7, 125.1, 119.9, 71.0, 53.9, 46.0, 41.9, 36.7, 29.2, 25.7, 25.5, 23.9, 20.4, 17.7, 14.0; IR (thin film): $\widetilde{v} 3399,2961,2925,1592,1413$, 1311,1241, 960, $787 \mathrm{~cm}^{-1}$; HRMS (ESI): $\mathrm{m} / z$ calculated for $\mathrm{C}_{20} \mathrm{H}_{30} \mathrm{~N}_{2} \mathrm{OSNa}^{+}[\mathrm{M}+\mathrm{Na}]^{+}: 369.1971$, found: 369.1972; $[\alpha]_{\mathrm{D}}^{26} 5.6(c$ $\left.1.0, \mathrm{CHCl}_{3}\right)$. 


\section{Transformations of Products}

\section{4-1. Hydrolysis of amide to methyl ester}

A solution of the thioamide (5a) $(32.7 \mathrm{mg}, 0.1 \mathrm{mmol})$ in $2 \mathrm{M} \mathrm{HCl}$ in $\mathrm{MeOH}(2 \mathrm{~mL})$ was heated by an oil bath in a sealed pressure tube at $60^{\circ} \mathrm{C}$ for $8 \mathrm{~h}$. After cooling to RT the volatiles were removed under reduced pressure (caution: evolution of $\mathrm{H}_{2} \mathrm{~S}$ !) and the residue was purified by flash chromatography with hexane/ethyl acetate (1/1) to provide $20.3 \mathrm{mg}(85 \%$, d.r. $>20 / 1$ ) of 6 .

\section{Methyl (2S,3R)-3-hydroxy-2-methyl-5-phenylpentanoate (6)}<smiles>CC(=O)C(O)C(O)CCc1ccccc1</smiles>

Reaction performed according to the general procedure afforded $620.3 \mathrm{mg}$ (85\%, d.r. >20/1).

Colorless oil; ${ }^{1} \mathrm{H}$ NMR (400 MHz, $\left.300 \mathrm{~K}, \mathrm{CDCl}_{3}\right)$ : $\delta$ 7.31-7.26 (m, 2H), 7.23-7.15 (m, 3H), 3.95-3.88 (m, 1H), $3.70(\mathrm{~s}, 3 \mathrm{H}), 2.86(\mathrm{ddd}, J=14.8,9.9,5.3 \mathrm{~Hz}, 1 \mathrm{H}), 2.66(\mathrm{ddd}, J=13.8,9.6,6.9 \mathrm{~Hz}, 1 \mathrm{H}), 2.60-$ $2.51(\mathrm{~m}, 2 \mathrm{H}), 1.81(\mathrm{dtd}, J=14.8,9.5,5.3 \mathrm{~Hz}, 1 \mathrm{H}), 1.67$ (dddd, $J=13.8,10.2,6.9,3.6 \mathrm{~Hz}, 1 \mathrm{H}), 1.19$ (d, $J=7.2 \mathrm{~Hz}, 3 \mathrm{H}) ;{ }^{13} \mathrm{C}$ NMR $\left(101 \mathrm{MHz}, 300 \mathrm{~K}, \mathrm{CDCl}_{3}\right)$ : $\delta$ 176.5, 141.8, 128.5, 128.4, 125.9, 71.0, 51.8, 44.3, 35.5, 32.3, 10.8; IR (KBr): $\widetilde{v} 3432,2964,1716,1455,1434,1200,1042,913,745,699 \mathrm{~cm}^{-1}$; HRMS (ESI): $m / z$ calculated for $\mathrm{C}_{13} \mathrm{H}_{18} \mathrm{O}_{3} \mathrm{Na}^{+}\left[\mathrm{M}+\mathrm{Na}^{+}\right.$: 245.1148, found: 245.1150; $[\alpha]_{\mathrm{D}}^{26} 11.4\left(c 0.9, \mathrm{CHCl}_{3}\right.$, sample derived from aldol adduct with $95 \%$ ee).

\section{4-2. Desulfurization of thioamide to amide [3]}

A solution of the thioamide (5a) $(20.0 \mathrm{mg}, 0.06 \mathrm{mmol})$ in $\mathrm{EtOH}(2 \mathrm{~mL})$ was added $\mathrm{ZrCl}_{4}(14.0 \mathrm{mg}, 0.06 \mathrm{mmol})$ and $\mathrm{H}_{2} \mathrm{O}_{2}$ (30\% aqueous solution, $0.24 \mathrm{mmol}$ ) at RT. After strring for 1 hour at same tempreture, reaction mixture was added EtOAc $(20 \mathrm{ml})$. The dilluted solution was washed by water $(10 \mathrm{~mL} \times 3)$ and dired by $\mathrm{Na}_{2} \mathrm{SO}_{4}$. The filtrate was concentrated under reduced pressure and the residual product was purified by silica gel column chromatography (ethyl acetate/hexane $=$ 2/1) to give amide.

\section{(2S,3R)-1-(2,3-dihydro-1H-pyrrolo[2,3-b]pyridin-1-yl)-3-hydroxy-2-methyl-5-phenylpentan-1-one (7)}

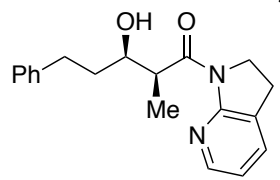

Reaction performed according to the general procedure afforded $715.5 \mathrm{mg}(82 \%$, d.r. >20/1). Colorless oil; ${ }^{1} \mathrm{H}$ NMR (400 MHz, $\left.300 \mathrm{~K}, \mathrm{CDCl}_{3}\right)$ : $\delta$ 8.15-8.05 (m,1H), 7.54-7.46 (m, 1H), 5.32-7.15 (m, $5 \mathrm{H}), 6.92(\mathrm{dd}, J=5.1,7.4 \mathrm{~Hz}, 1 \mathrm{H}), 4.75-3.75(\mathrm{~m}, 5 \mathrm{H}), 3.15-298(\mathrm{~m}, 2 \mathrm{H}), 2.95-2.85(\mathrm{~m}, 1 \mathrm{H}), 2.75-2.64(\mathrm{~m}$, $1 \mathrm{H}), 1.99-1.87(\mathrm{~m}, 1 \mathrm{H}), 1.85-1.72(\mathrm{~m}, 1 \mathrm{H}), 1.23(\mathrm{~d}, J=7.0 \mathrm{~Hz}, 3 \mathrm{H}) ;{ }^{13} \mathrm{C} \mathrm{NMR}\left(101 \mathrm{MHz}, 300 \mathrm{~K}, \mathrm{CDCl}_{3}\right)$ : ठ 177.0, 155.6, 146.0, 142.5, 134.0, 128.7, 128.4, 126.7, 125.8, 118.6, 71.7, 46.0, 42.0, 35.3, 32.5, 24.3, 11.1; IR (KBr): $\widetilde{v} 3460,1634$, 1122, 734, $700 \mathrm{~cm}^{-1}$; HRMS (ESI): $m / z$ calculated for $\mathrm{C}_{19} \mathrm{H}_{23} \mathrm{~N}_{2} \mathrm{O}_{2}+[\mathrm{M}+\mathrm{H}]^{+}: 311.1754$, found: $311.1759 ;[\alpha]_{\mathrm{D}}^{23}-39.1(c 0.9$, $\mathrm{CHCl}_{3}$, sample derived from aldol adduct with $94 \%$ ee). 


\section{Crystal structure analysis}

\section{8-1. Solid state structure of $\mathbf{2 a}$}

Single crystals of 2a were obtained by vapor diffusion from dichloromethane/pentane at RT. A suitable crystal was selected and the sample was measured on a Rigaku R-AXIS RAPIS II diffractometer using multi-layer mirror monochromated $\mathrm{Cu}-\mathrm{K} \alpha$ radiation. The data were collected at $93 \mathrm{~K}$. Refined structure and crystallographic parameters are summarized in Supplementary Table S1 and Supplementary Fig. S1. CCDC 1966091 contains the supplementary crystallographic data for $\mathbf{2 a}$.

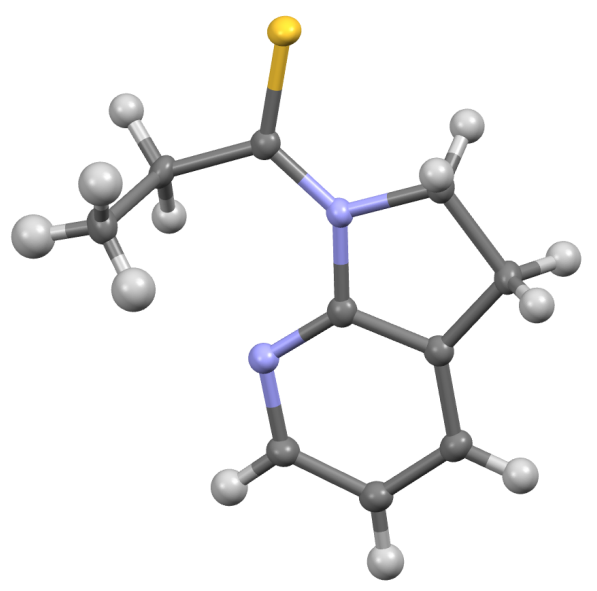

Figure S1. Structure of $2 \mathbf{a}$ in the solid state. Color code: yellow: sulfur; light blue: nitrogen; gray: carbon; white: hydrogen.
Table S1. Selected crystal data of $2 a$.

\begin{tabular}{|c|c|}
\hline Empirical Formula & $\mathrm{C}_{10} \mathrm{H}_{12} \mathrm{~N}_{2} \mathrm{~S}$ \\
\hline Formula Weight & 192.28 \\
\hline Crystal System & triclinic \\
\hline Crystal size $/ \mathrm{mm}^{3}$ & $0.1 \times 0.05 \times 0.05$ \\
\hline Space group & P-1 \\
\hline \multicolumn{2}{|l|}{ Lattice Parameters } \\
\hline $\mathrm{a}$ & $5.05620(10) \AA$ \\
\hline $\mathrm{b}$ & $8.0590(3) \AA$ \\
\hline c & $11.8737(4) \AA$ \\
\hline$\alpha$ & $99.650(3)^{\circ}$ \\
\hline$\beta$ & $96.833(3)^{\circ}$ \\
\hline$\gamma$ & $94.699(2)^{\circ}$ \\
\hline $\mathrm{V}$ & $471.02(3) \AA^{3}$ \\
\hline$Z$ value & 2 \\
\hline $\mathrm{R}_{1}$ & 0.0614 \\
\hline$w R_{2}$ & 0.1816 \\
\hline$D_{\text {calc }}$ & $1.356 \mathrm{~g} / \mathrm{cm}^{3}$ \\
\hline $\mathrm{F}_{000}$ & 204.00 \\
\hline
\end{tabular}


Product 5a (5 mg) was derivatized with 3,5-dichlorobenzoic acid $(4.4 \mathrm{mg})$ by Steglich esterification protocol (DMAP $2.8 \mathrm{mg} / \mathrm{EDCI} 4.4 \mathrm{mg} / \mathrm{DCM} 0.3 \mathrm{~mL}$ ). After aqueous workup, crystals of $\mathbf{5} \mathbf{a}^{\prime}$ were obtained by slow evaporation from hexane at RT. A suitable crystal was selected and the sample was measured on a Rigaku R-AXIS RAPIS II diffractometer using multi-layer mirror monochromated $\mathrm{Cu}-\mathrm{K} \alpha$ radiation. The data were collected at $93 \mathrm{~K}$. Refined structure and crystallographic parameters are summarized in Supplementary Table S2 and Supplementary Fig. S2. CCDC 1966092 contains the supplementary crystallographic data for $\mathbf{5 a}$ '.

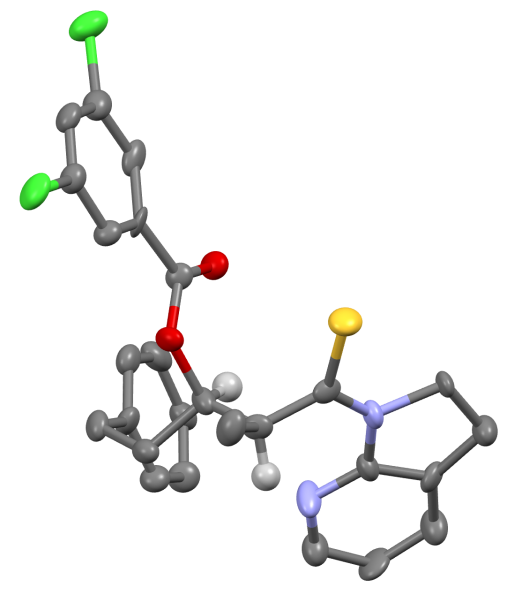

Figure S2. Structure of $\mathbf{5 a} \mathbf{a}^{\prime}$ in the solid state. Color code: yellow: sulfur; light blue: nitrogen; red: oxygen; green: chlorine; gray: carbon; white: hydrogen. Non-essential hydrogen atoms were removed for clarity.
Table S2. Selected crystal data of $\mathbf{5} \mathbf{a}^{\prime}$.

\begin{tabular}{|c|c|}
\hline Empirical Formula & $\left(\mathrm{C}_{26} \mathrm{H}_{24} \mathrm{Cl}_{2} \mathrm{~N}_{2} \mathrm{O}_{2} \mathrm{~S}\right)$ \\
\hline Formula Weight & 3995.44 \\
\hline Crystal System & monoclinic \\
\hline Crystal size $/ \mathrm{mm}^{3}$ & $0.2 \times 0.1 \times 0.02$ \\
\hline Space group & $\mathrm{P} 21$ \\
\hline \multicolumn{2}{|l|}{ Lattice Parameters } \\
\hline $\mathrm{a}$ & $34.6873(4) \AA$ \\
\hline $\mathrm{b}$ & $7.90870(10) \AA$ \\
\hline c & 36.3956(4) Å \\
\hline$\alpha$ & $90^{\circ}$ \\
\hline$\beta$ & $105.0330(10)^{\circ}$ \\
\hline$\gamma$ & $90^{\circ}$ \\
\hline $\mathrm{V}$ & $9642.8(2) \AA^{3}$ \\
\hline $\mathrm{Z}$ value & 2 \\
\hline Flack parameter & $0.036(6)$ \\
\hline $\mathrm{R}_{1}$ & 0.1145 \\
\hline$w R_{2}$ & 0.3013 \\
\hline$D_{\text {calc }}$ & $1.376 \mathrm{~g} / \mathrm{cm}^{3}$ \\
\hline$F_{000}$ & 4160.00 \\
\hline
\end{tabular}


Procedure to make NMR sample of enolate using mesitylcopper

A flame-dried $20 \mathrm{~mL}$ test tube equipped with a magnetic stirring bar and a 3-way glass stopcock were charged with thioamide 2 (0.1 mmol), (S,S)-Ph-BPE (55.7 mg, $0.11 \mathrm{mmol}, 1.1$ eq.) and mesitylcopper $(20.1 \mathrm{mg}$, $0.11 \mathrm{~mol}, 1.1 \mathrm{eq}$.$) in a$ glove box. To the mixture was added THF- $d_{8}(1.0 \mathrm{~mL})$ via syringe with a stainless-steel needle at room temperature. The resulting solution was stirred for $5 \mathrm{~min}$ at same temperature and part of solution was transferred to well-died and Arfilled NNR tube to be analyzed immediately.

All three thioamides (2a, 2e and 2f) delivered Z-enolates only, which were confirmed by NOE (Figure S7, S8 and S9).

Procedure to make NMR sample of cationic complex

A flame-dried $20 \mathrm{~mL}$ test tube equipped with a magnetic stirring bar and a 3-way glass stopcock were charged with thioamide $2(0.1 \mathrm{mmol}),(S, S)-\mathrm{Ph}-\mathrm{BPE}\left(55.7 \mathrm{mg}, 0.11 \mathrm{mmol}, 1.1\right.$ eq.) and $[\mathrm{Cu}(\mathrm{MeCN}) 4] \mathrm{PF}_{6}(41.0 \mathrm{mg}, 0.11 \mathrm{~mol}, 1.1 \mathrm{eq}$.) in a glove box. To the mixture was added THF- $d_{8}(1.0 \mathrm{~mL})$ via syringe with a stainless-steel needle at room temperature. The resulting solution was stirred for $20 \mathrm{~min}$ at same temperature and part of solution was transferred to well-died and Arfilled NNR tube to be analyzed immediately.

Though $(S, S)-\mathrm{Ph}-\mathrm{BPE}$ and $\left[\mathrm{Cu}(\mathrm{MeCN})_{4}\right] \mathrm{PF}_{6}$ used were excess comparing to the thioamide, peaks of the unchelated free thioamide could be observed, which were labeled with red asterisk $\left(^{*}\right)$ in the Figure S4 and S5.

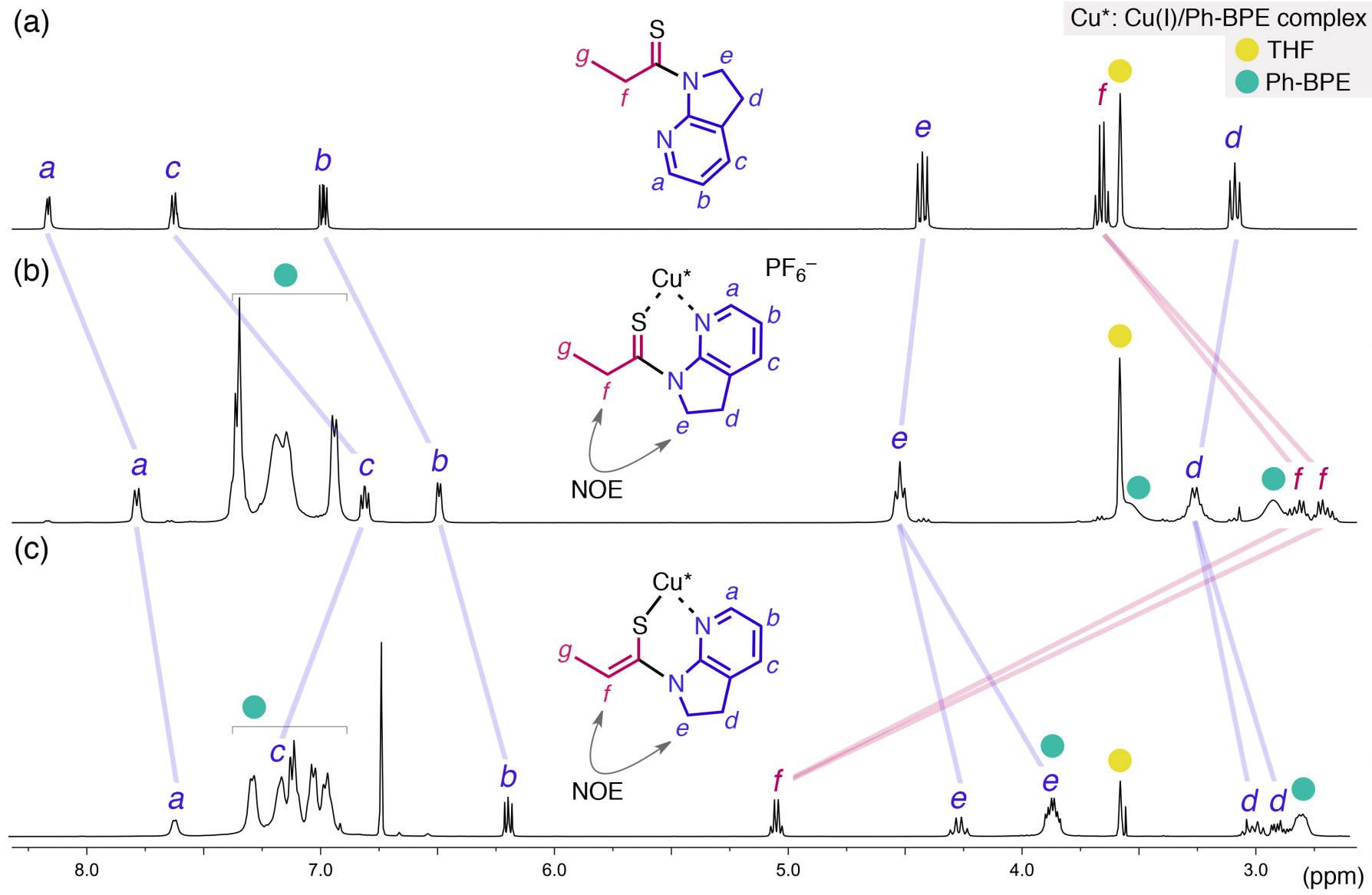

Figure S3. ${ }^{1} \mathrm{H}$ NMR spectra of 7 -azaindoline thiopropioamide $2 \mathrm{a}$ and $\mathrm{Cu}(\mathrm{I})$ complexes in THF- $d 8$. 

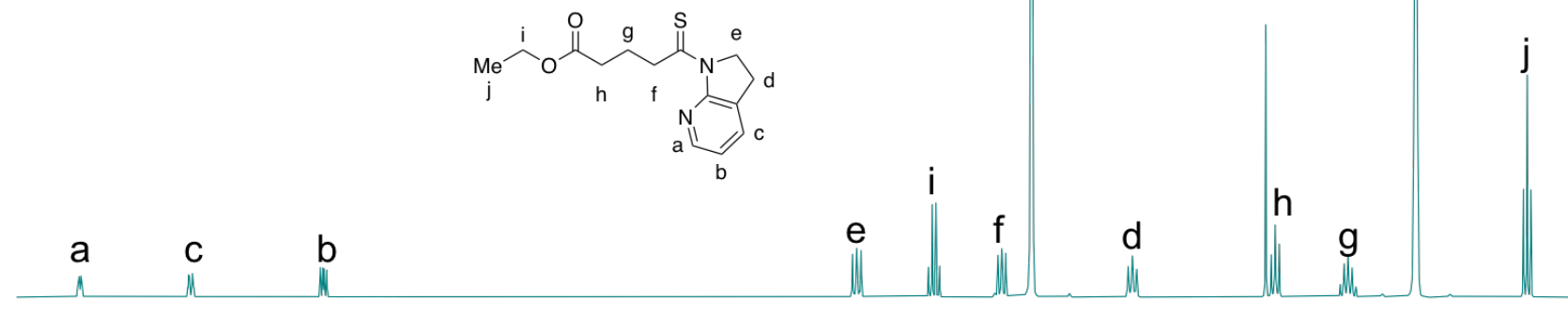

*: free thioamide
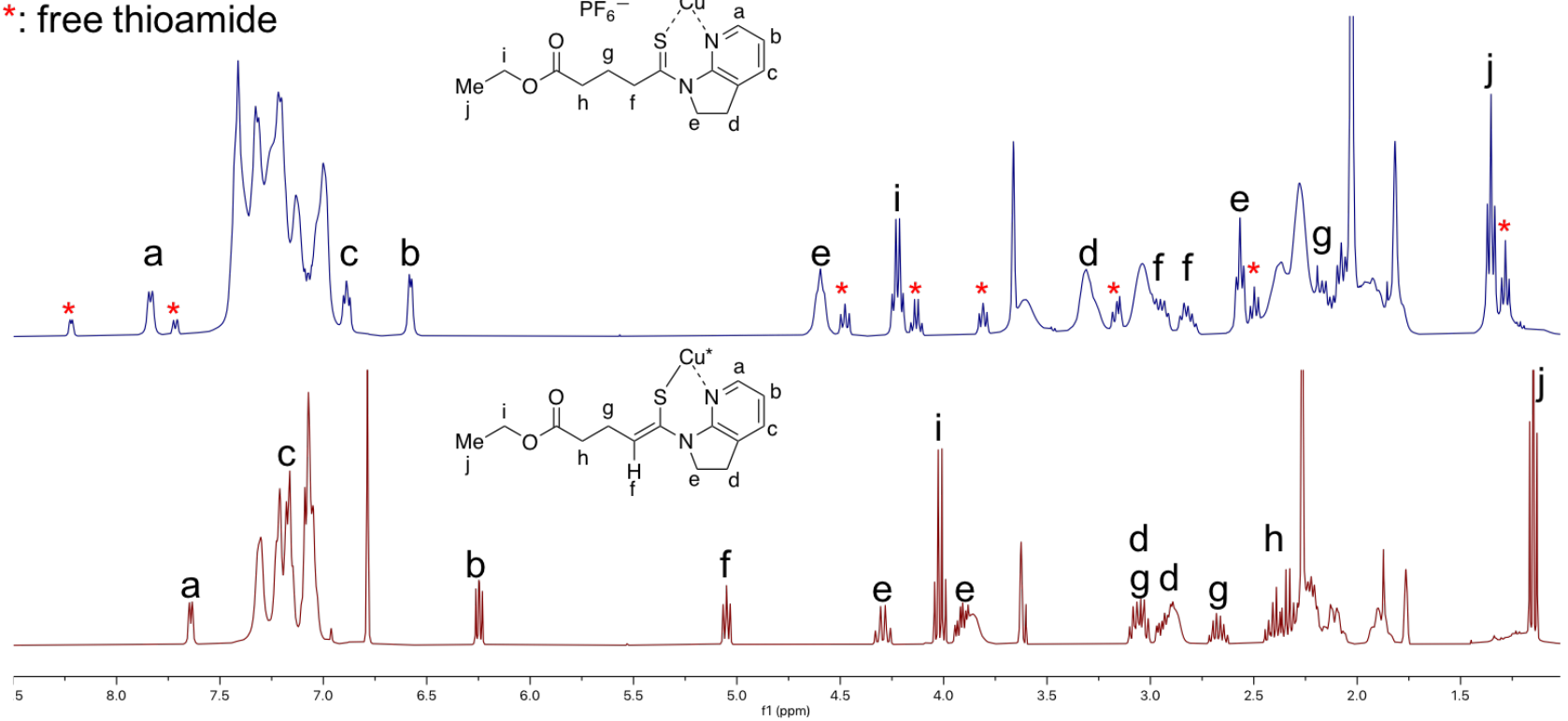

Figure S4. ${ }^{1} \mathrm{H}$ NMR spectra of 7 -azaindoline thiopropioamide $2 \mathbf{e}$ and $\mathrm{Cu}(\mathrm{I})$ complexes in THF- $d 8$.

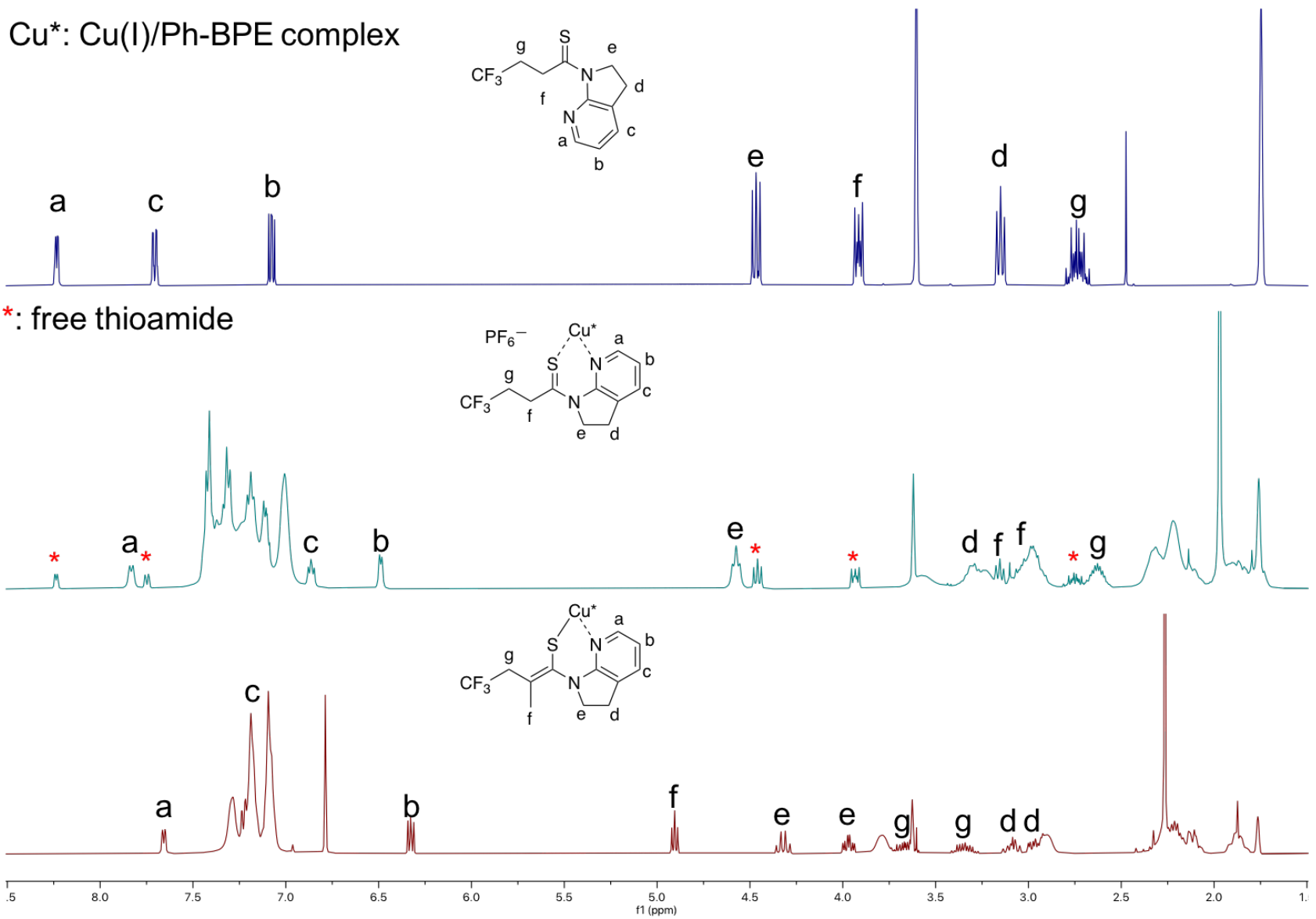

Figure S5. ${ }^{1} \mathrm{H}$ NMR spectra of 7 -azaindoline thiopropioamide $2 \mathrm{f}$ and $\mathrm{Cu}(\mathrm{I})$ complexes in THF- $d 8$. 


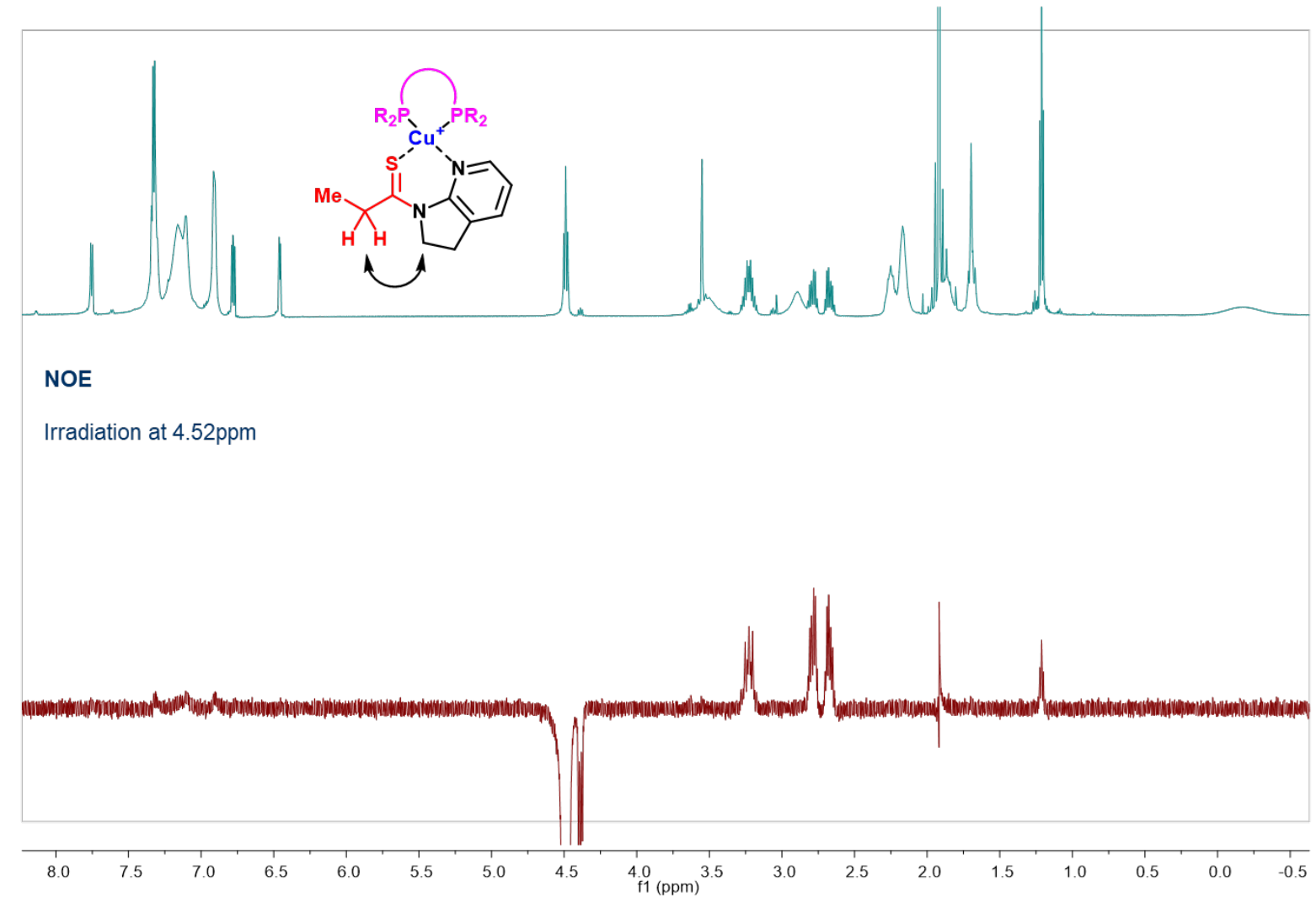

Figure S6. ${ }^{1} \mathrm{H}$ NMR spectra of cationic copper complex with 2a. NOE experiment confirms Z- configuration of amide bond.

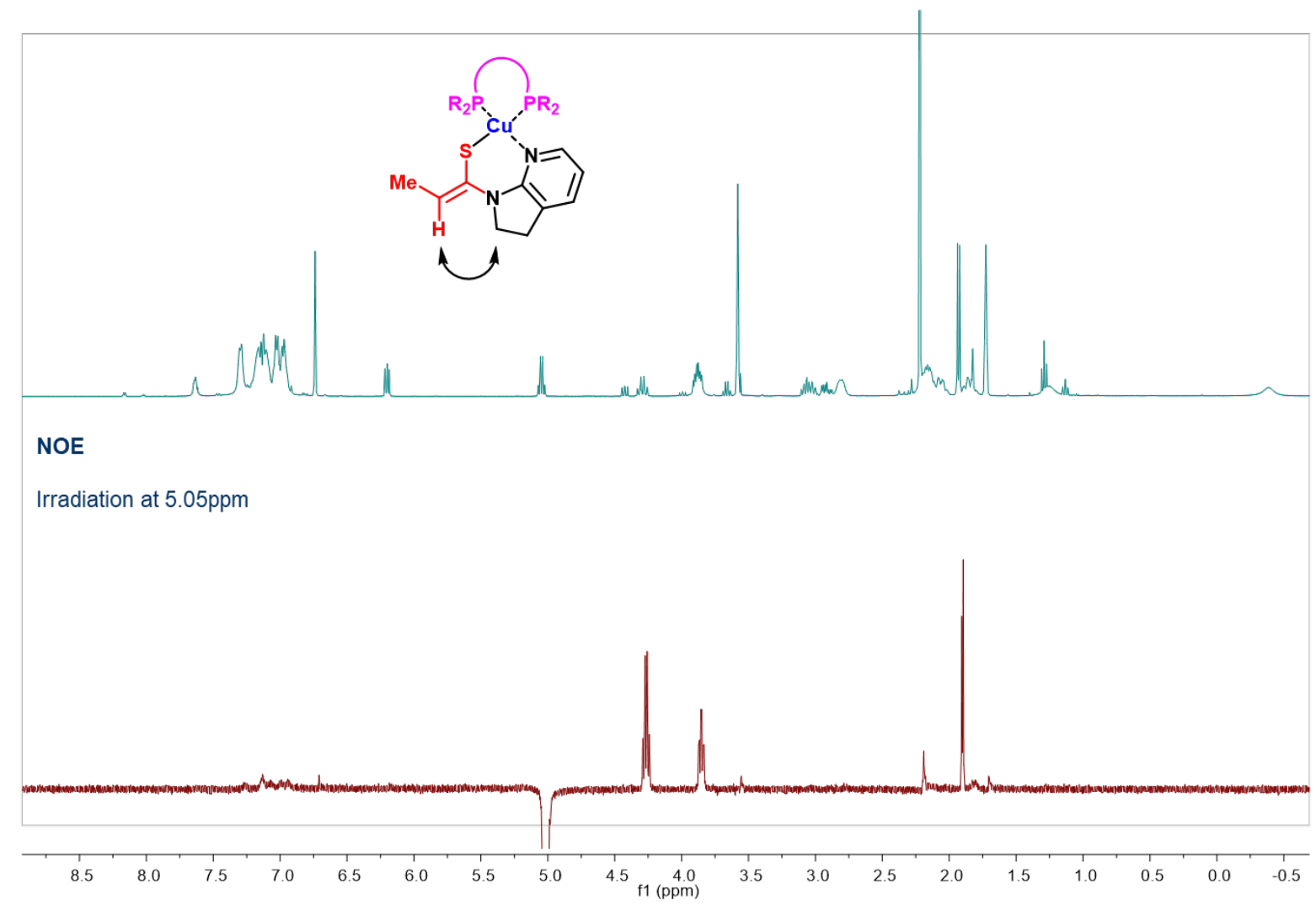

Figure S7. ${ }^{1} \mathrm{H}$ NMR spectra of copper thioenolate prepared in situ from thioamide $2 \mathbf{a},(S, S)-\mathrm{Ph}-\mathrm{BPE}$ and mesitylcopper. NOE spectrum confirms E-configuration of thioenolate. 


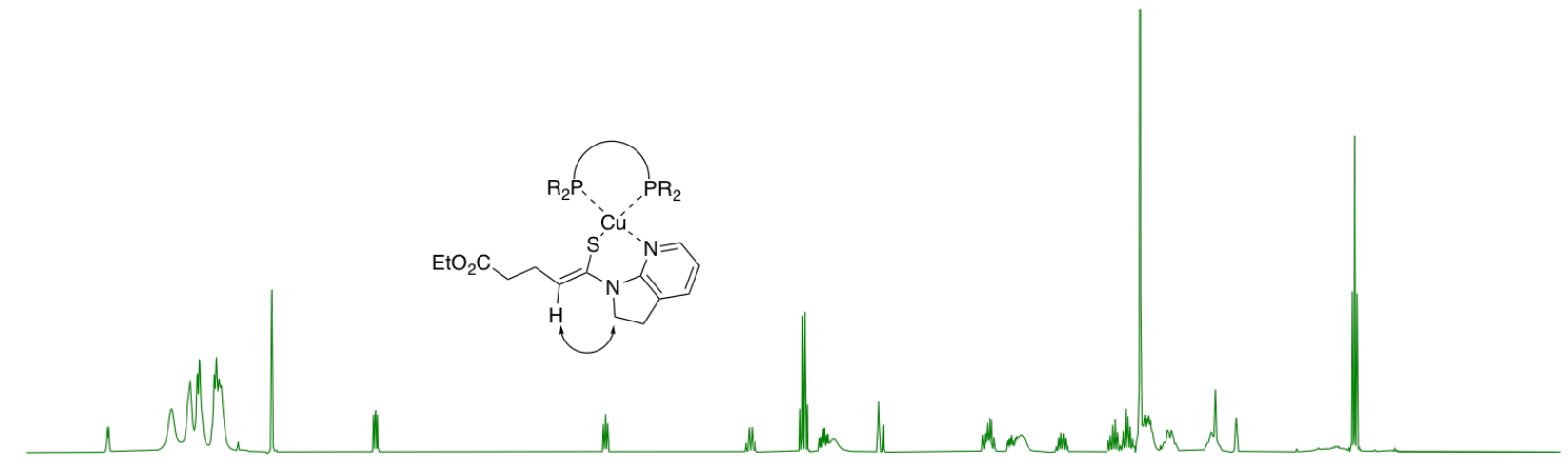

\section{NOE}

Irradiation at 5.01ppm

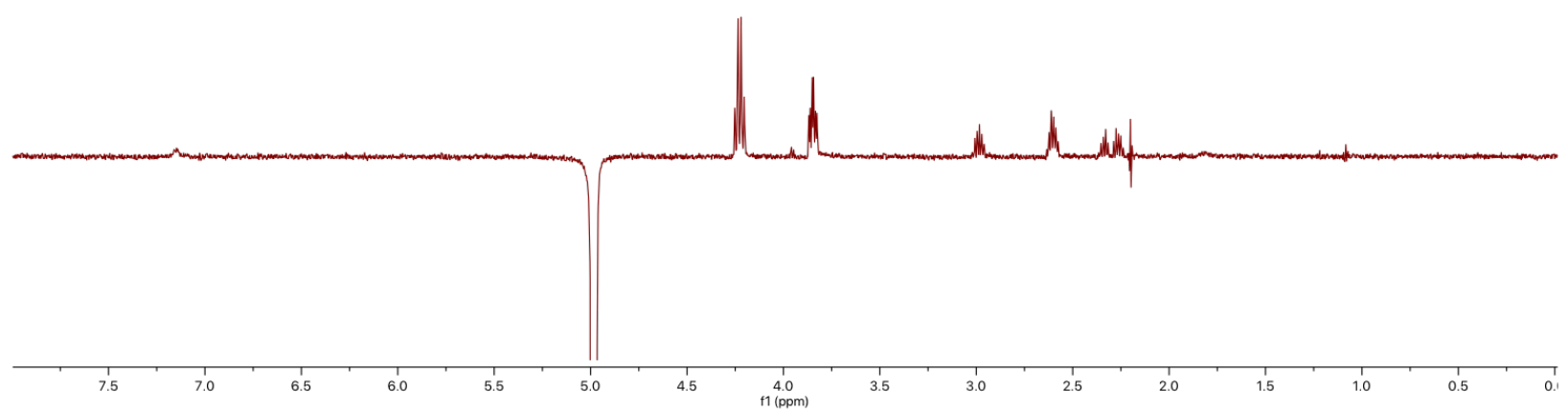

Figure S8. ${ }^{1} \mathrm{H}$ NMR spectra of copper thioenolate prepared in situ from thioamide 2e, $(S, S)-\mathrm{Ph}-\mathrm{BPE}$ and mesitylcopper. NOE spectrum confirms E-configuration of thioenolate.
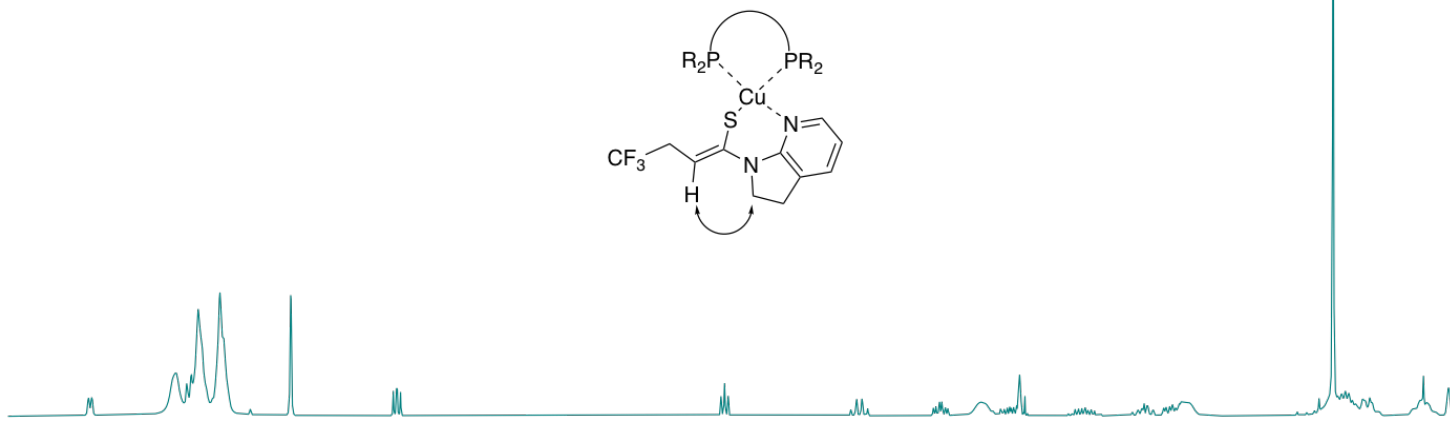

NOE

Irradiation at $4.87 \mathrm{ppm}$

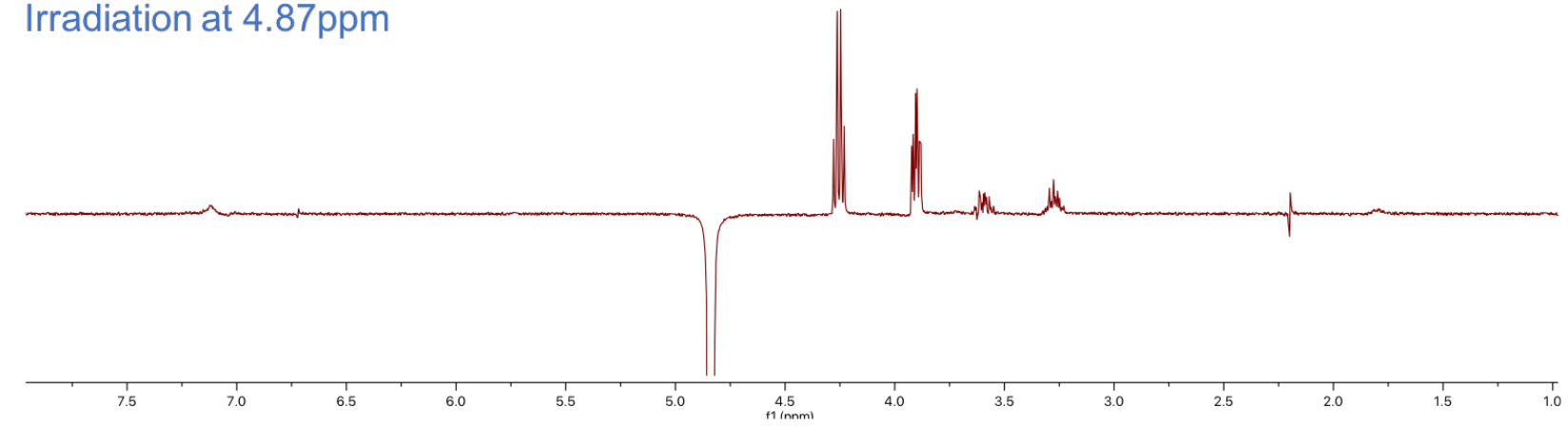

Figure S9. ${ }^{1} \mathrm{H}$ NMR spectra of copper thioenolate prepared in situ from thioamide $2 \mathbf{f},(S, S)-\mathrm{Ph}-\mathrm{BPE}$ and mesitylcopper. NOE spectrum confirms $E$-configuration of thioenolate. 

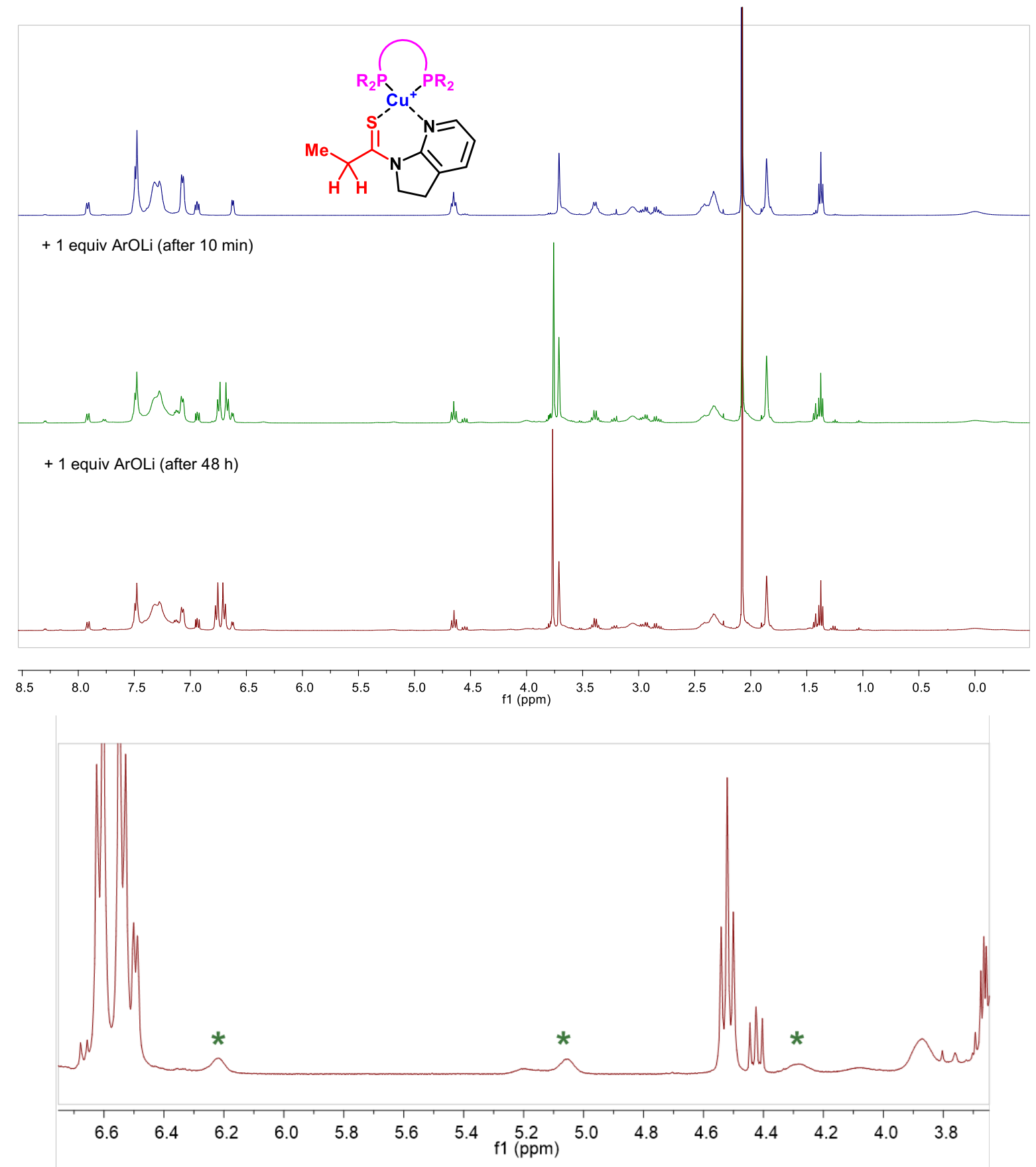

Figure S10. ${ }^{1} \mathrm{H}$ NMR spectrum of cationic copper complex after addition of 1 equiv of ArOLi (after $10 \mathrm{~min} \& 48 \mathrm{~h}$ after preparation). Protons assigned to enolate form are denoted with asterisks in the expansion.

\section{References}

[1] F. Arteaga Arteaga, Z. Liu, L. Brewitz, J. Chen, B. Sun, N. Kumagai, M. Shibasaki. Org. Lett. 2016, 18, 2391.

[2] Y. Ota, Z. Li, N. Kumagai, M. Shibasaki. Synlett 2019, 30, 620.

[3] K. Bahrami, M. M. Khodaei, Y. Tirandaz. Synthesis 2009, 3, 369. 
12. Spectra of New Compounds

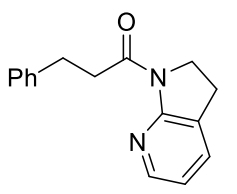

$F=F=0 \%$

等

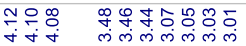

${ }^{1} \mathrm{H}$ NMR: S2c
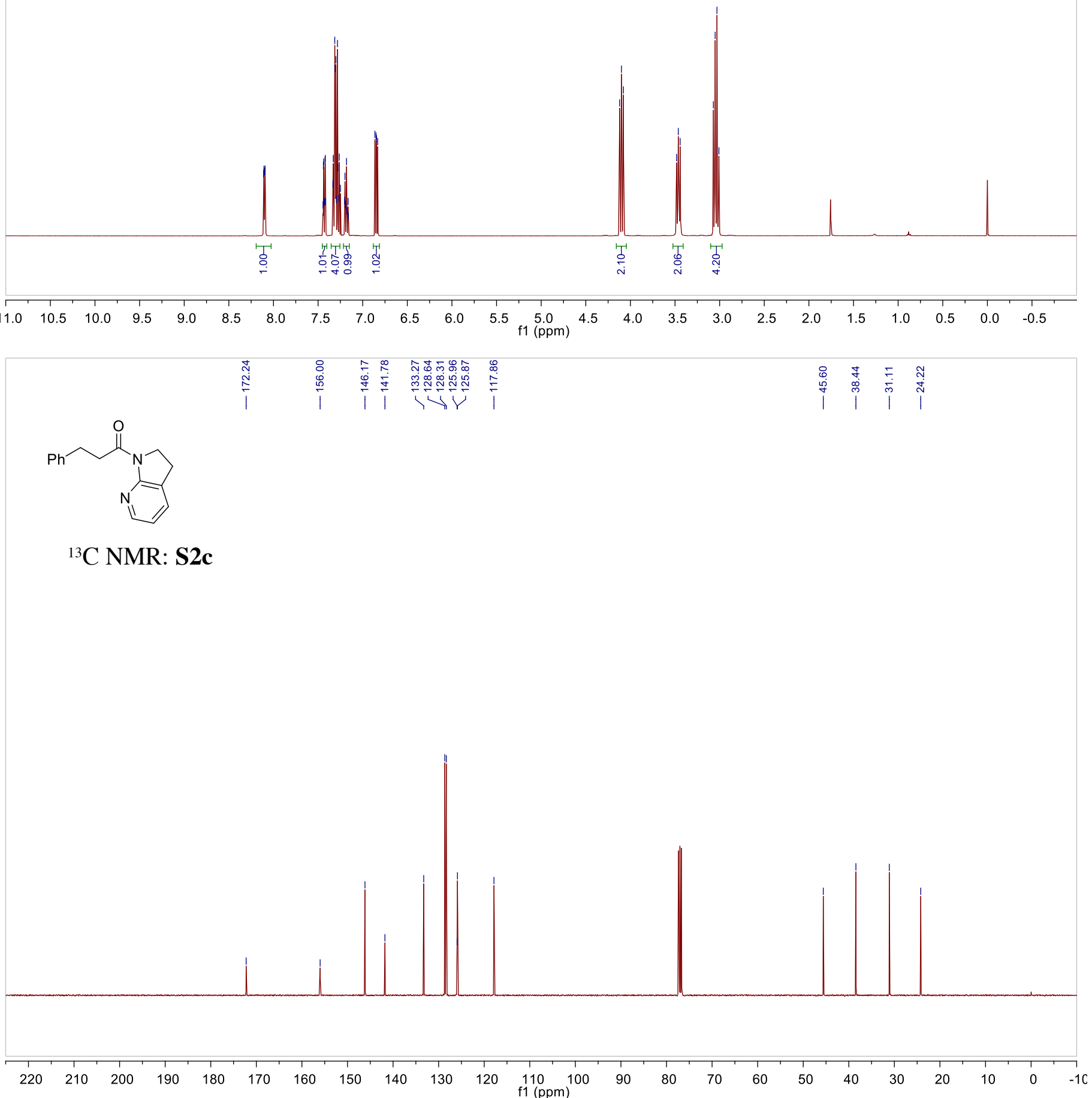

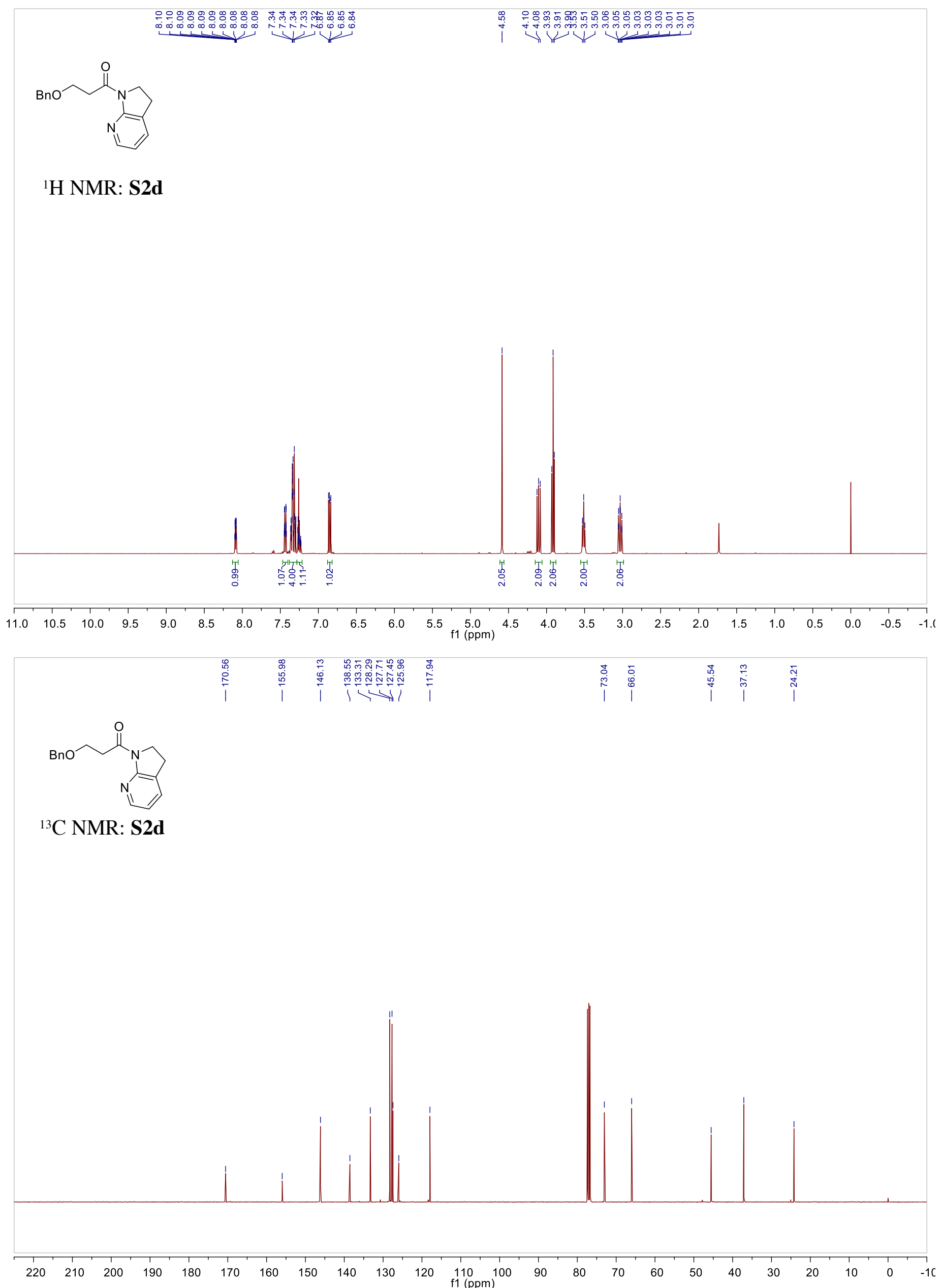

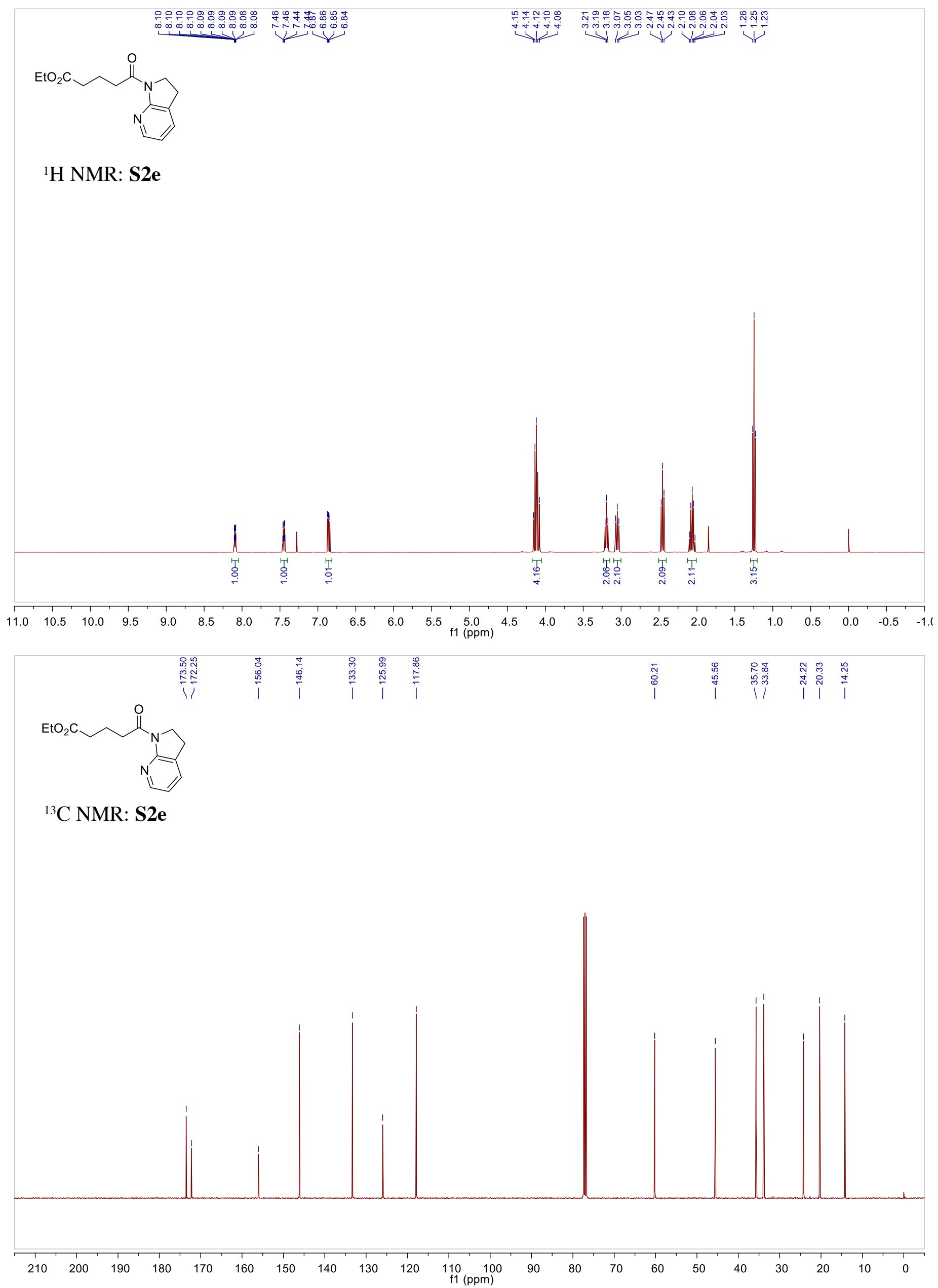

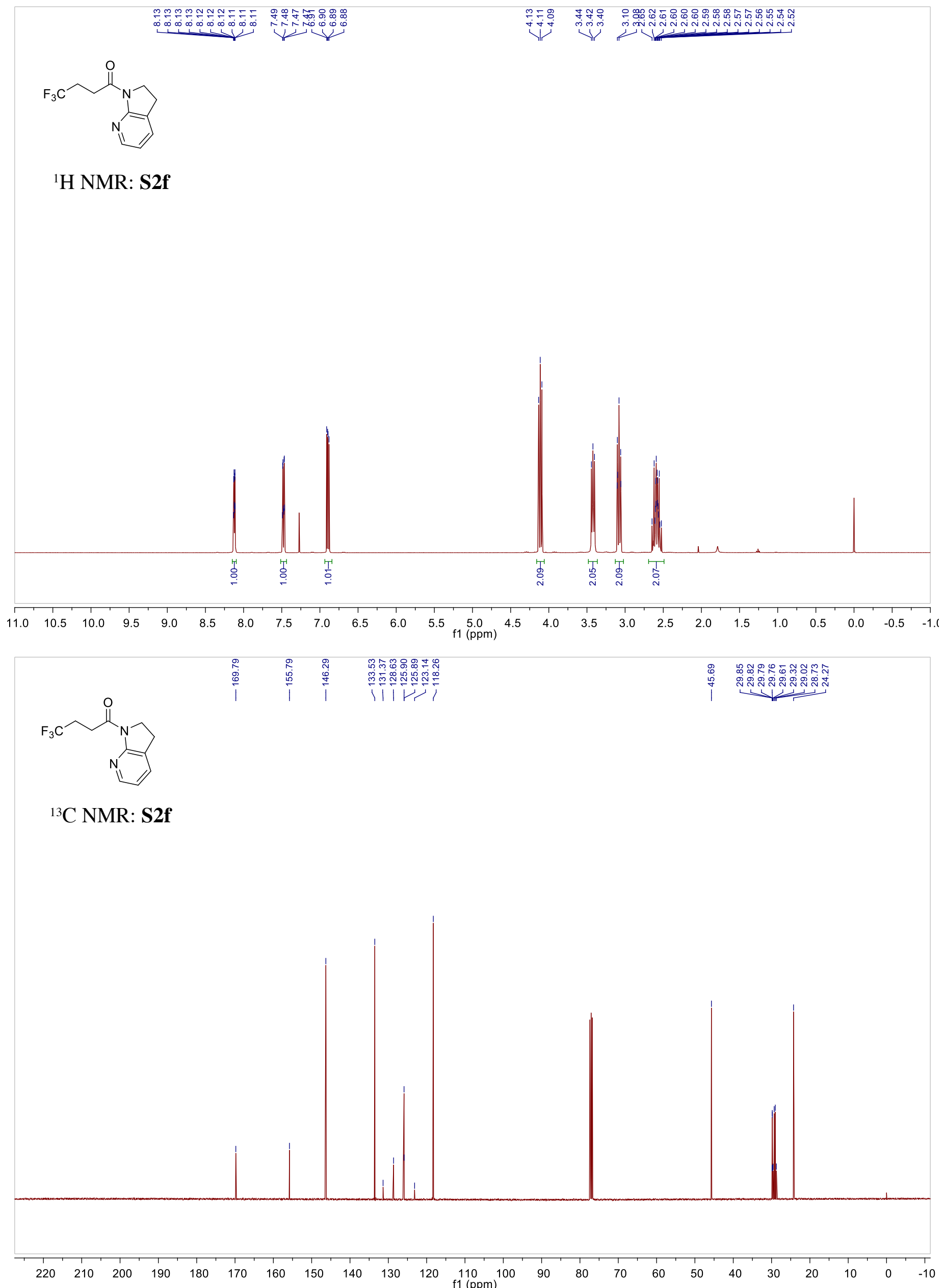


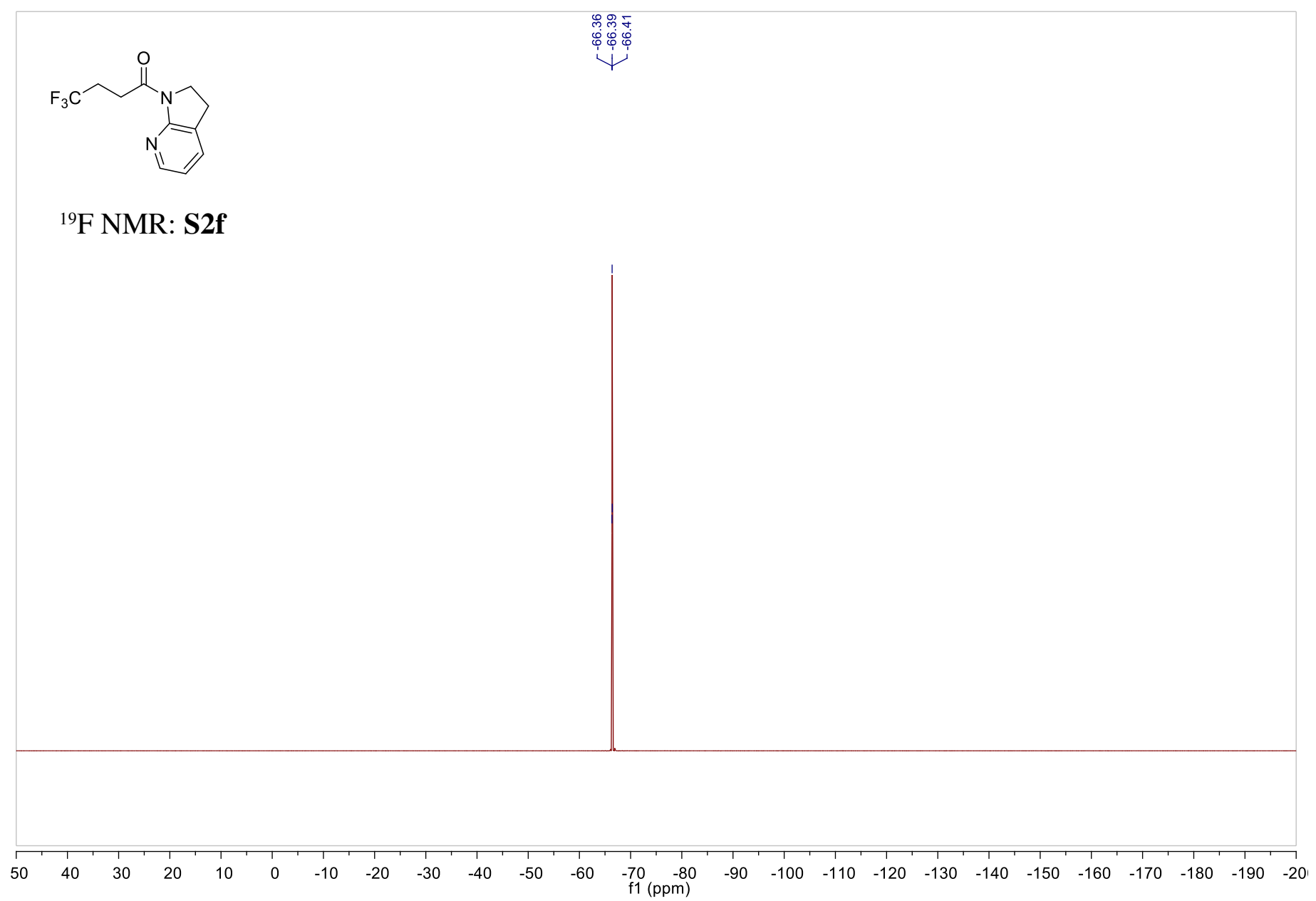



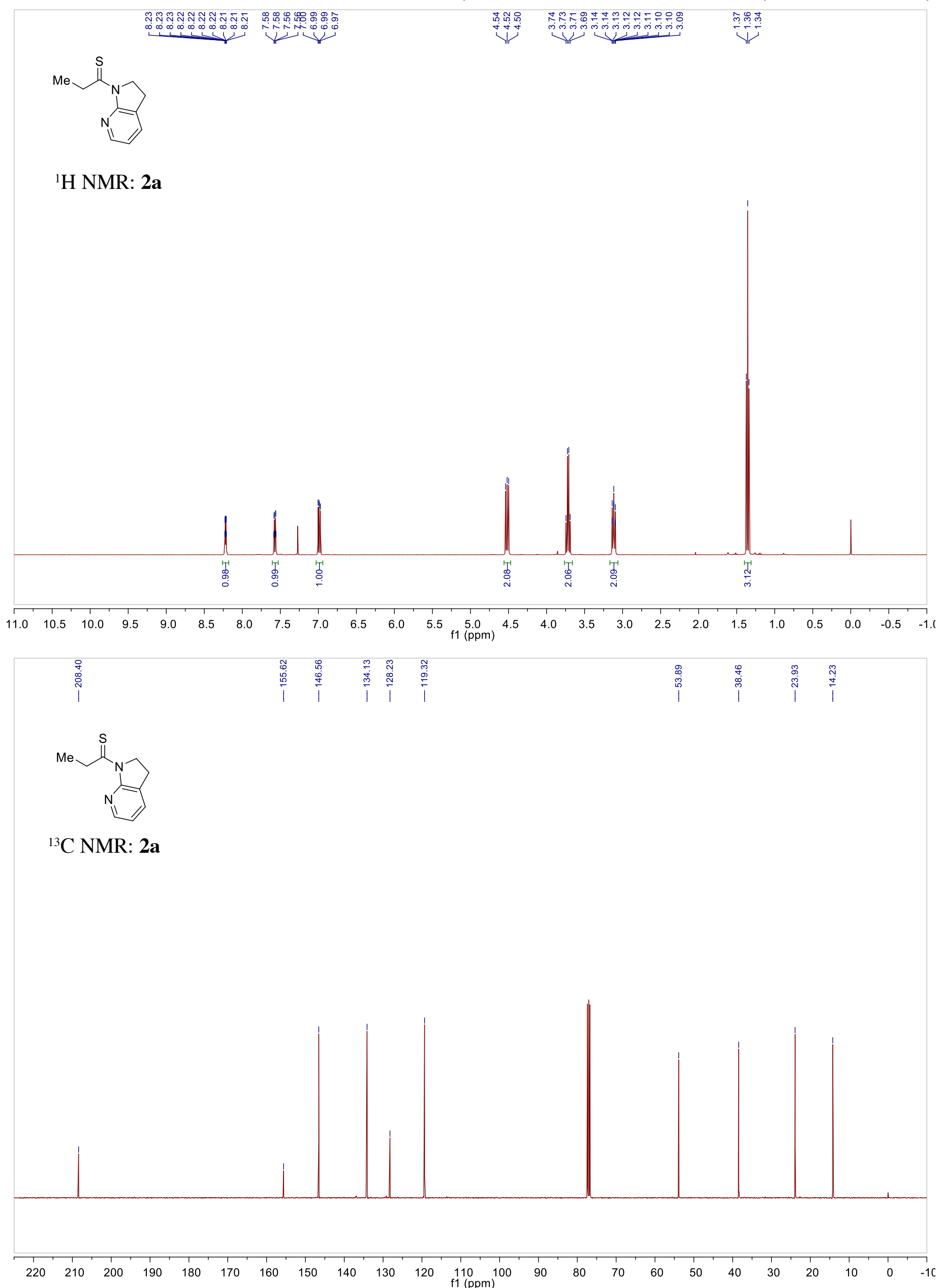

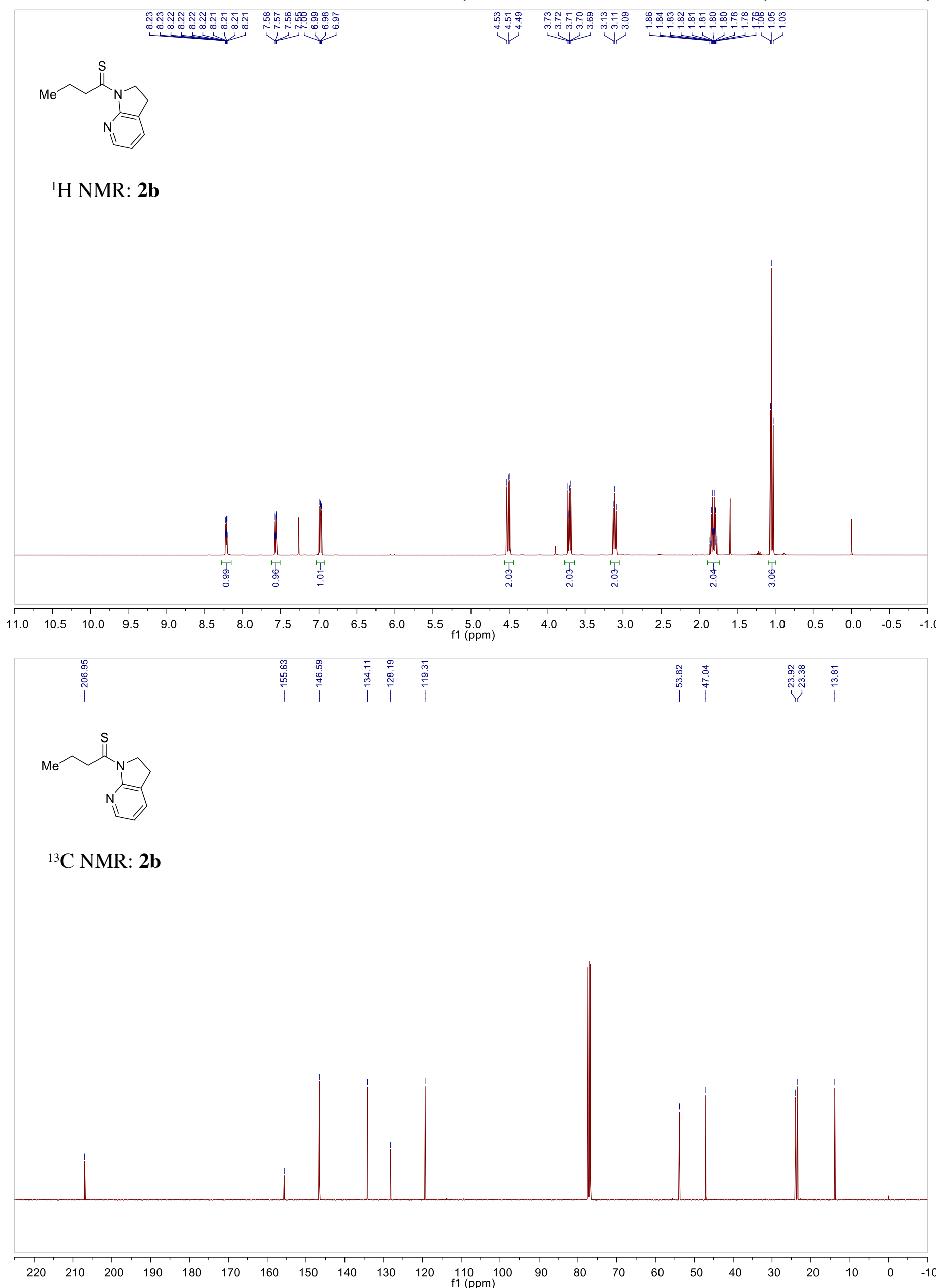

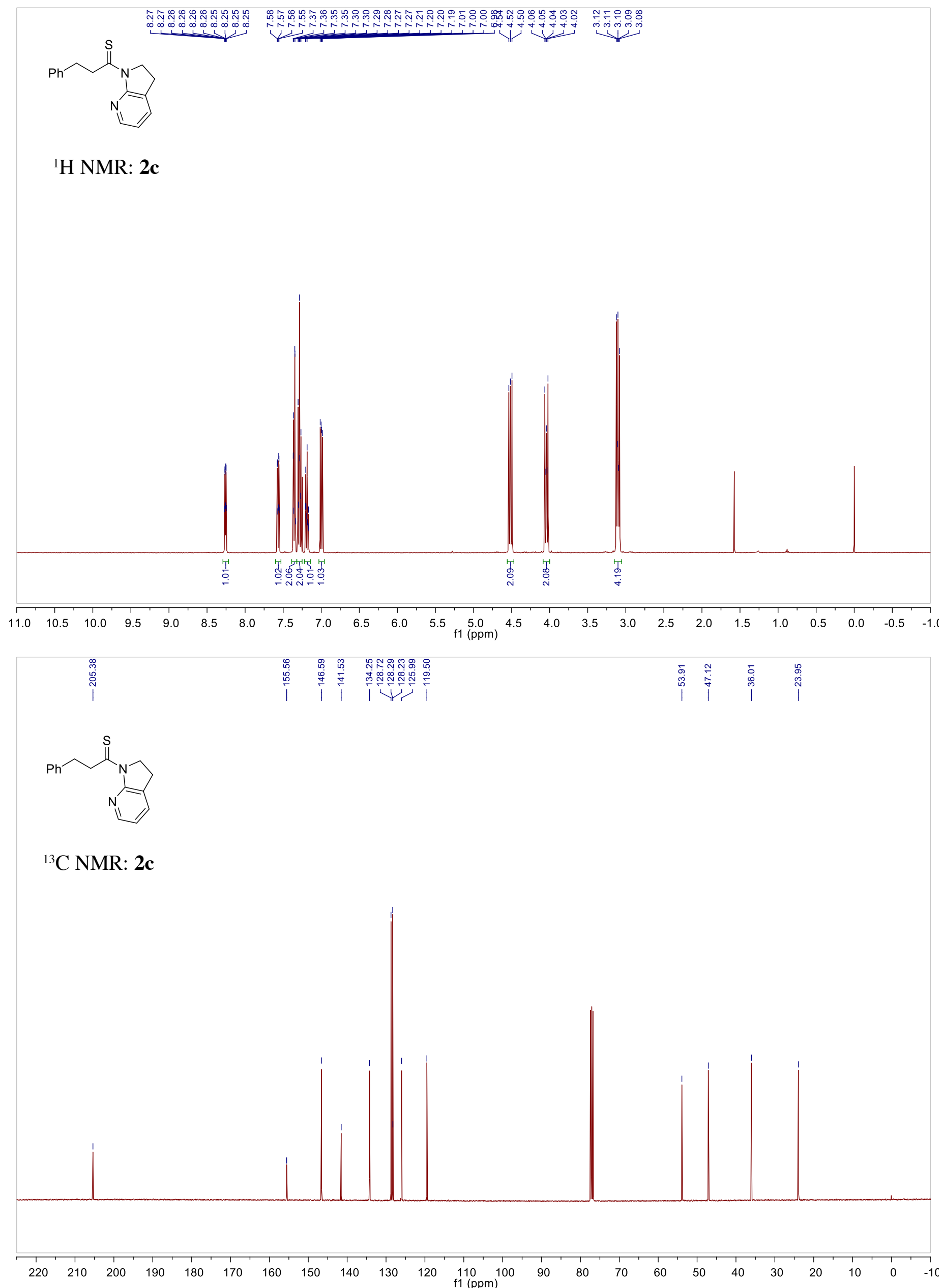

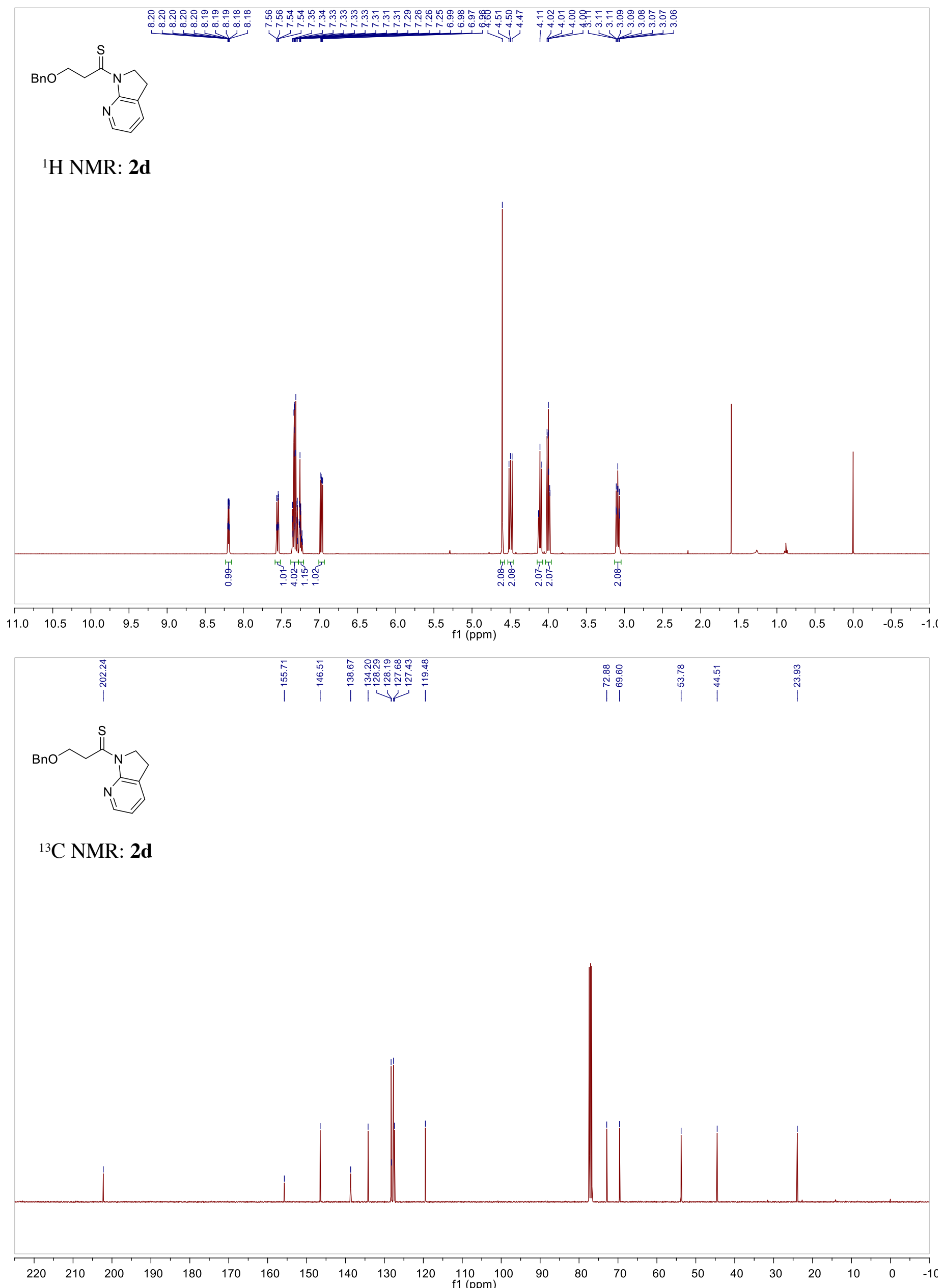

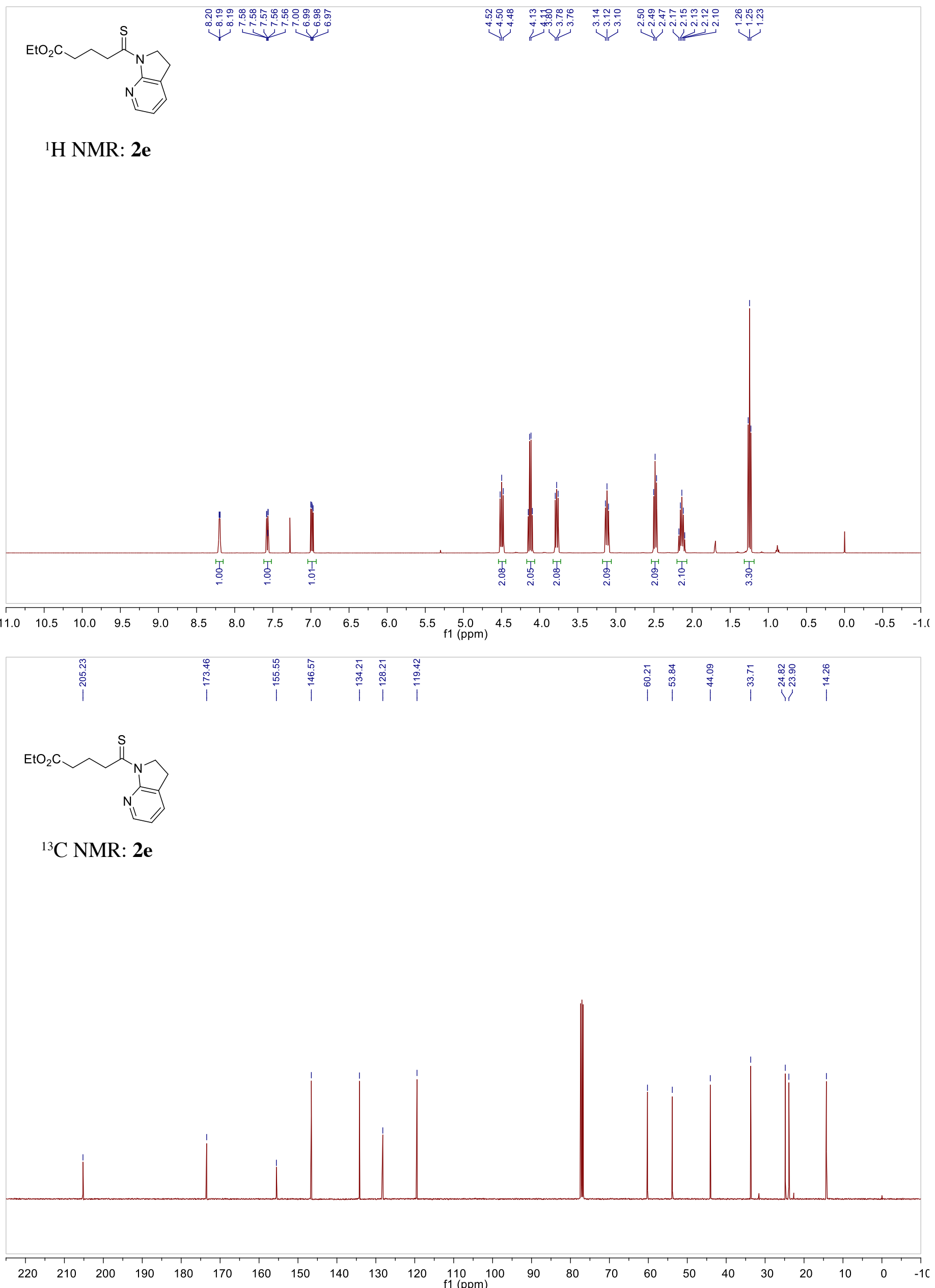

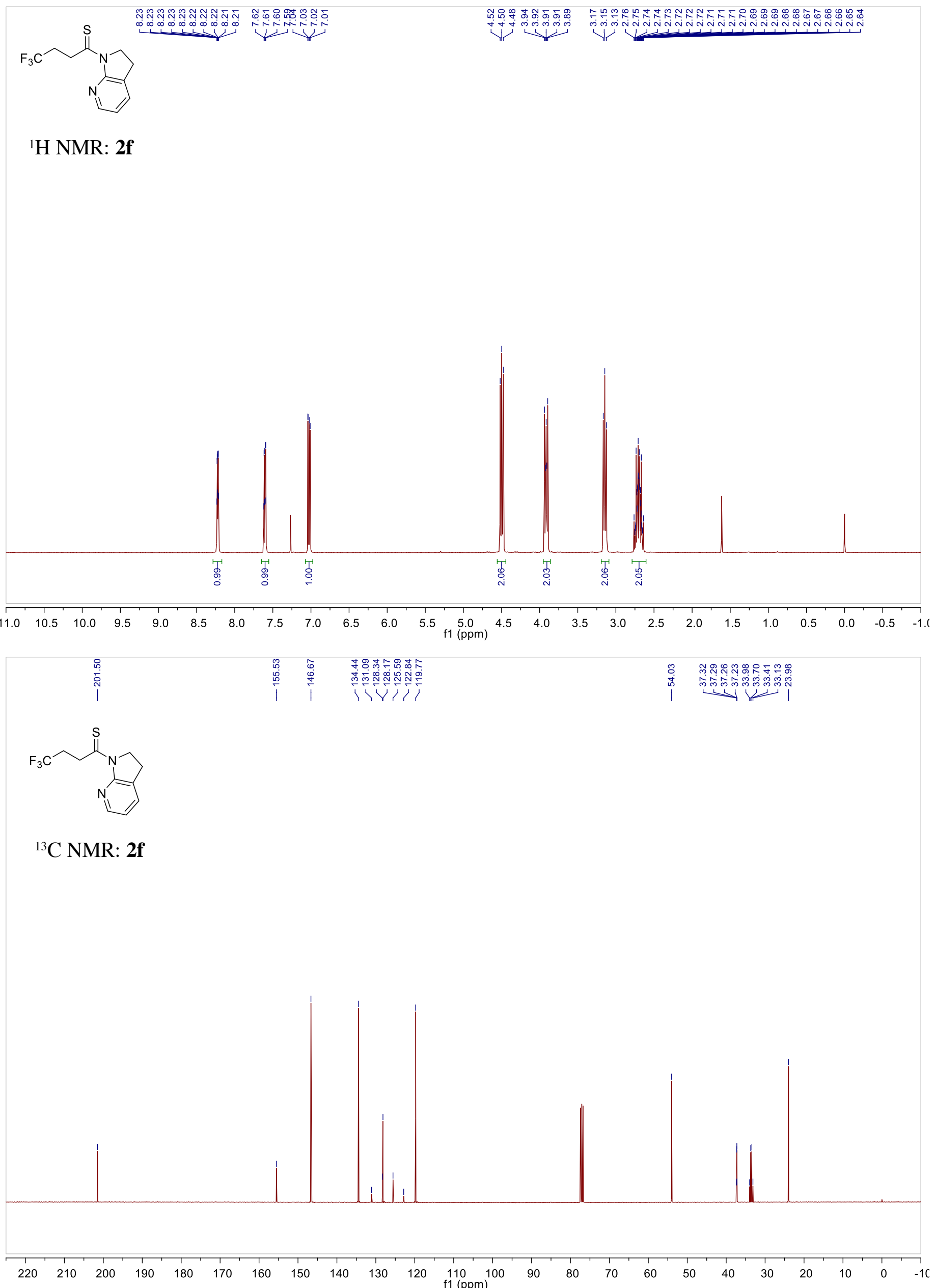


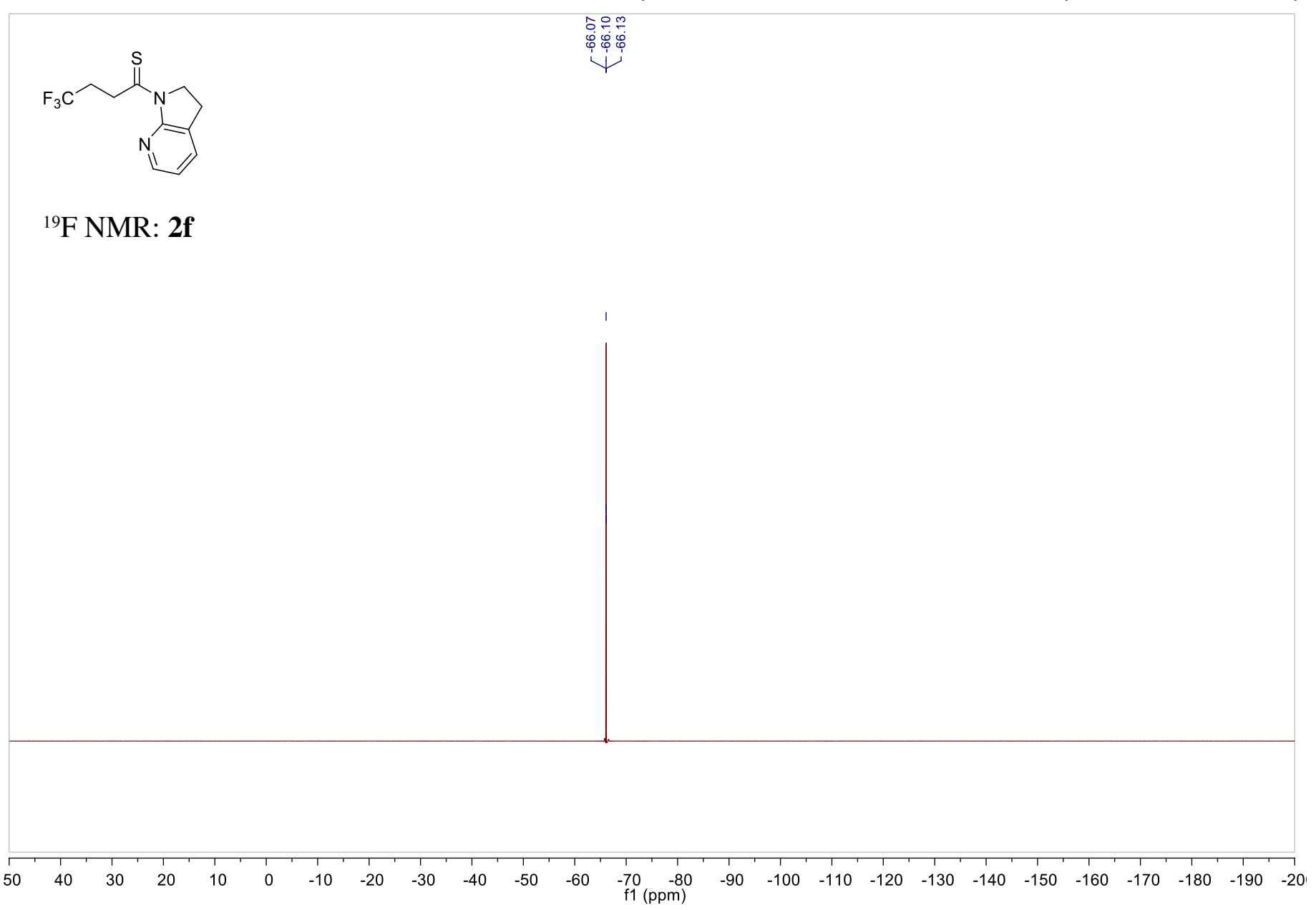


<smiles>[Y4]C(C(=S)N1CCc2cccnc21)C(O)CCc1ccccc1</smiles>

${ }^{1} \mathrm{H}$ NMR: 5a
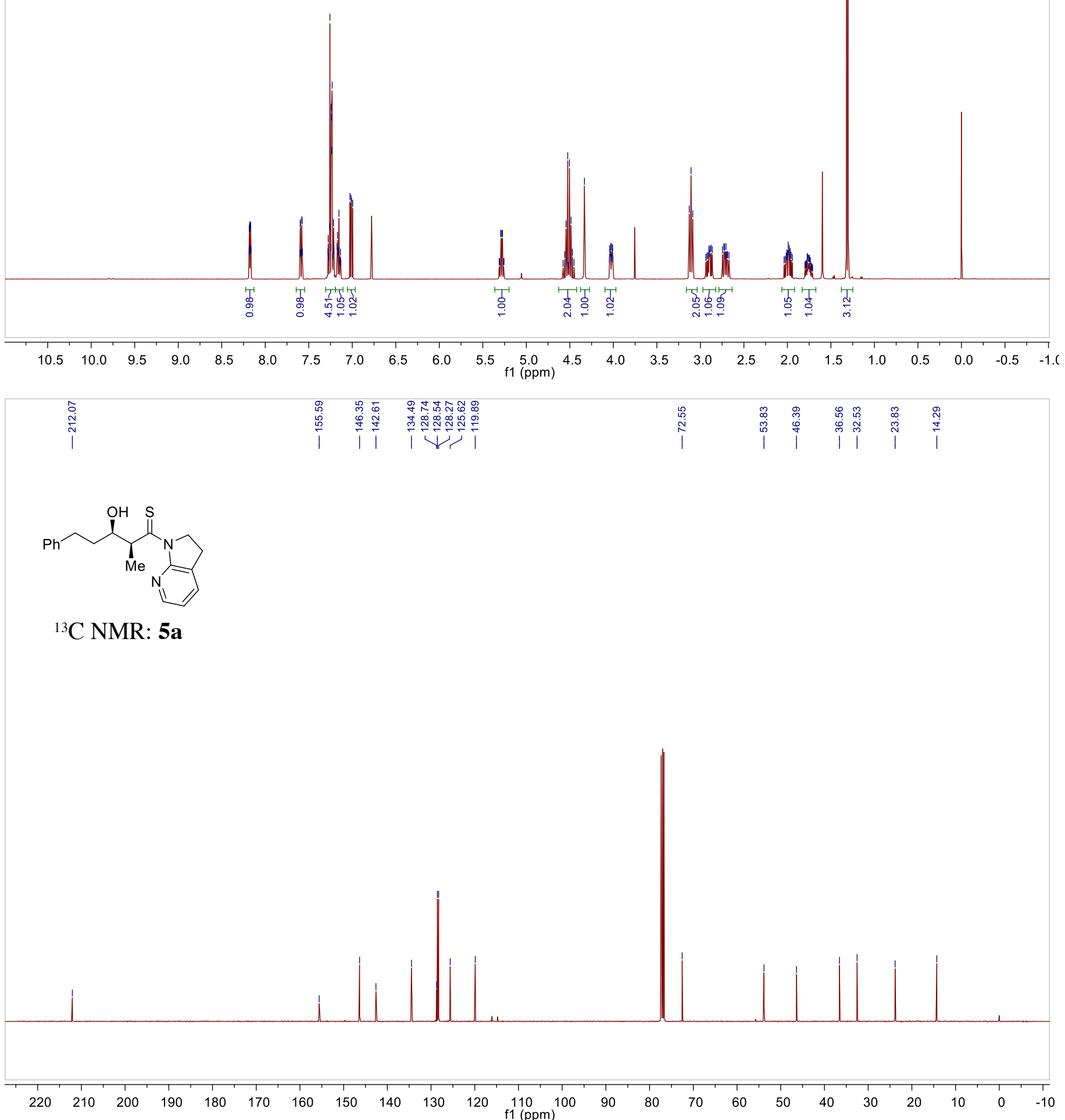

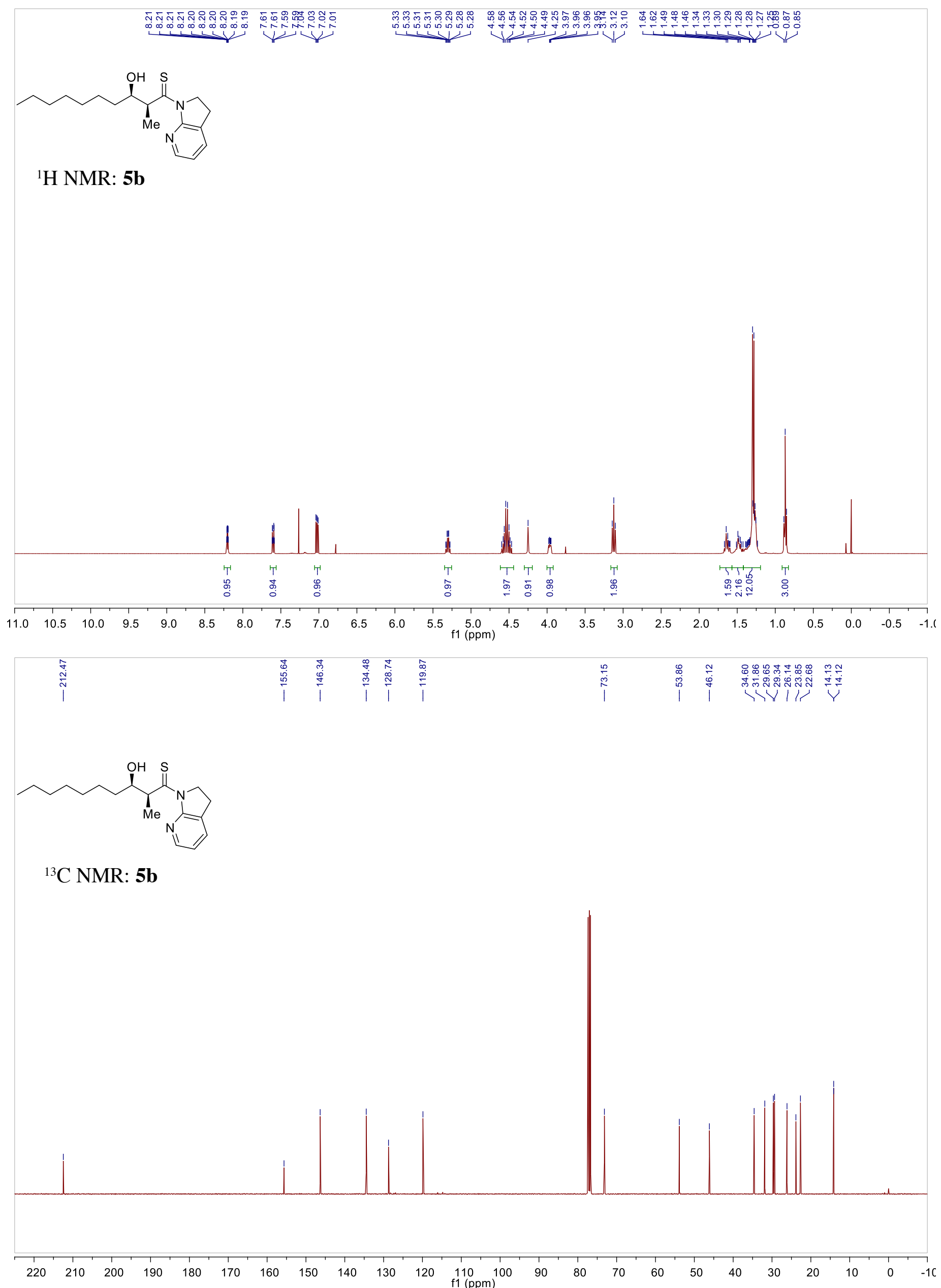
<smiles>CC(C)C[C@H](O)[C@H](C)C(=S)N1CCc2cccnc21</smiles>

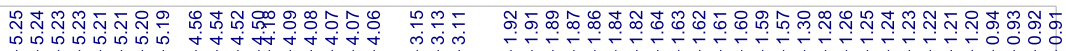

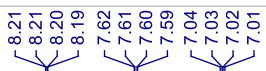

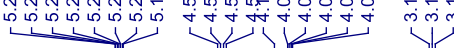

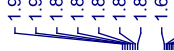

${ }^{1} \mathrm{H}$ NMR: 5c
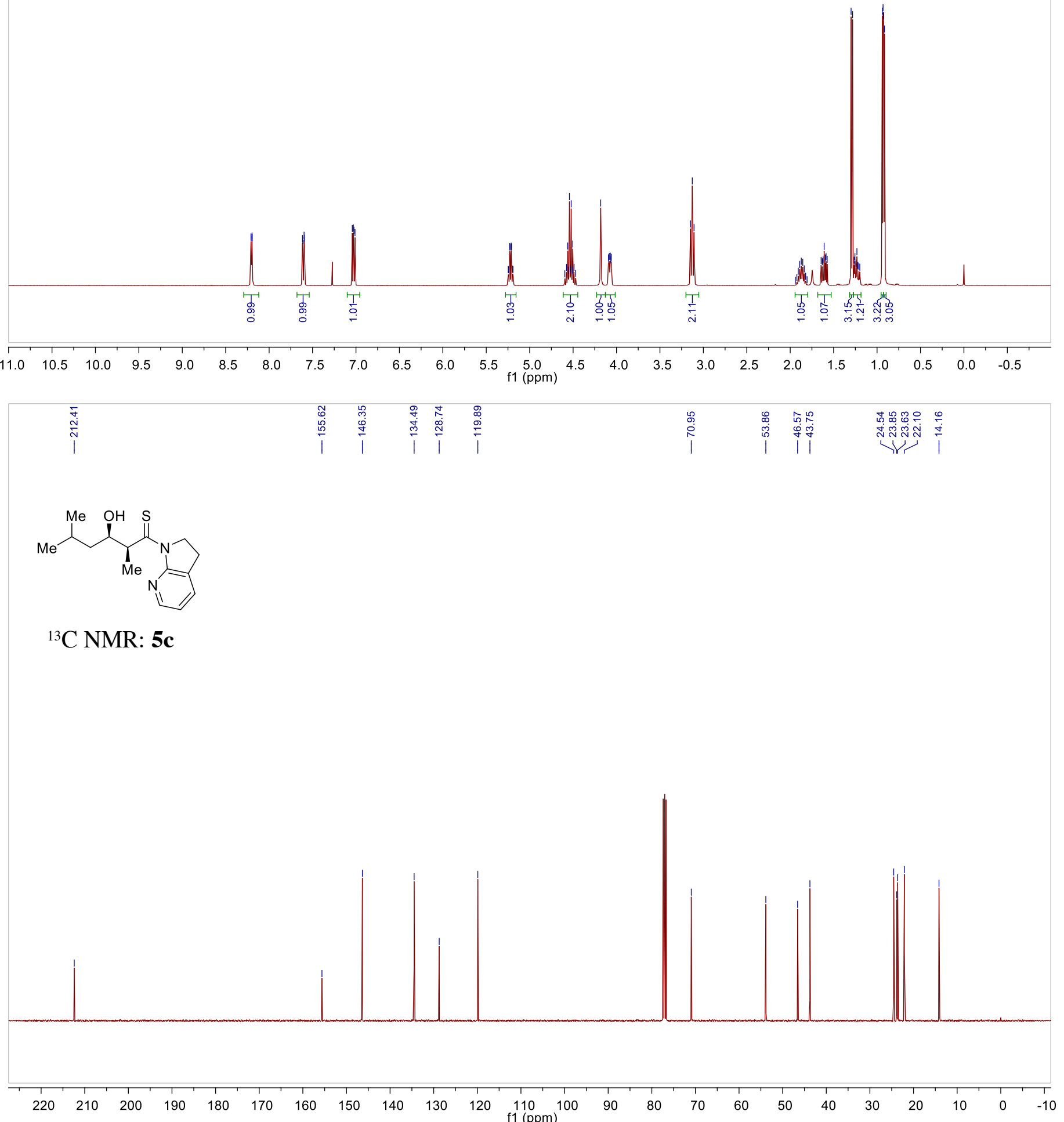


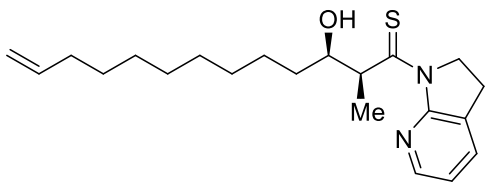

${ }^{1} \mathrm{H}$ NMR: 5d
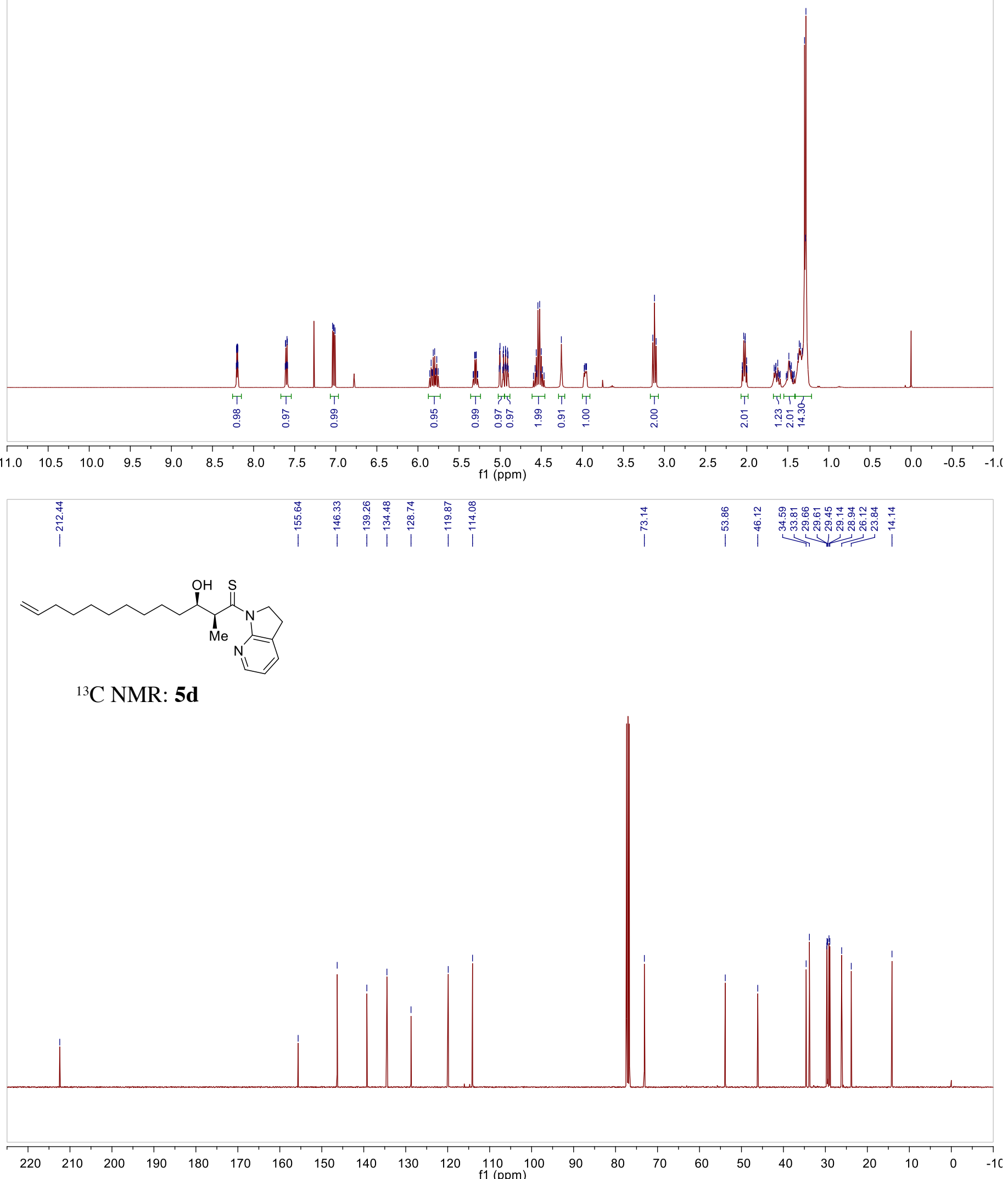
<smiles>C[C@@H](CCOc1ccccc1)[C@H](O)C(=S)N1CCc2cccnc21</smiles>

${ }^{1} \mathrm{H}$ NMR: 5e

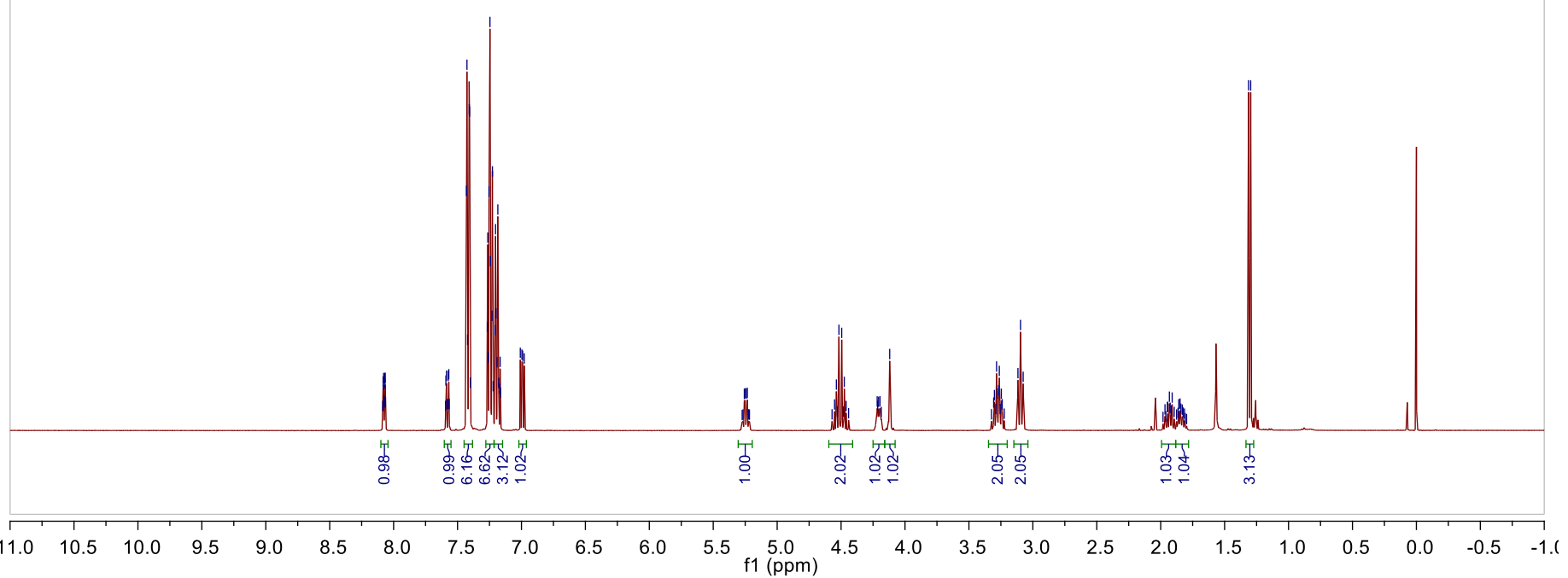

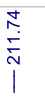<smiles>C[C@H](CCOc1ccccc1)[C@H](O)C(=S)N1CCc2cccnc21</smiles>

${ }^{13} \mathrm{C}$ NMR: 5e

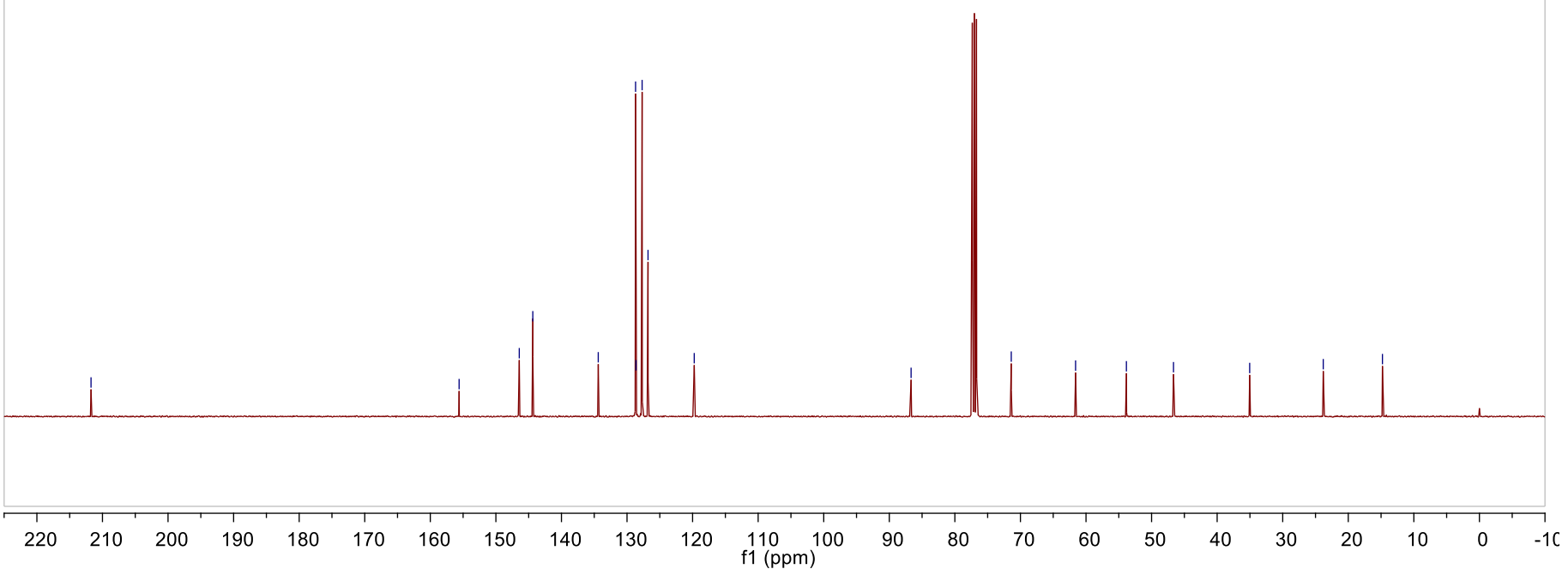


<smiles>CCOC(=O)CCCC[C@H](O)[C@H](C)C(=S)N1CCc2cccnc21</smiles>

${ }^{1} \mathrm{H}$ NMR: $\mathbf{5 f}$

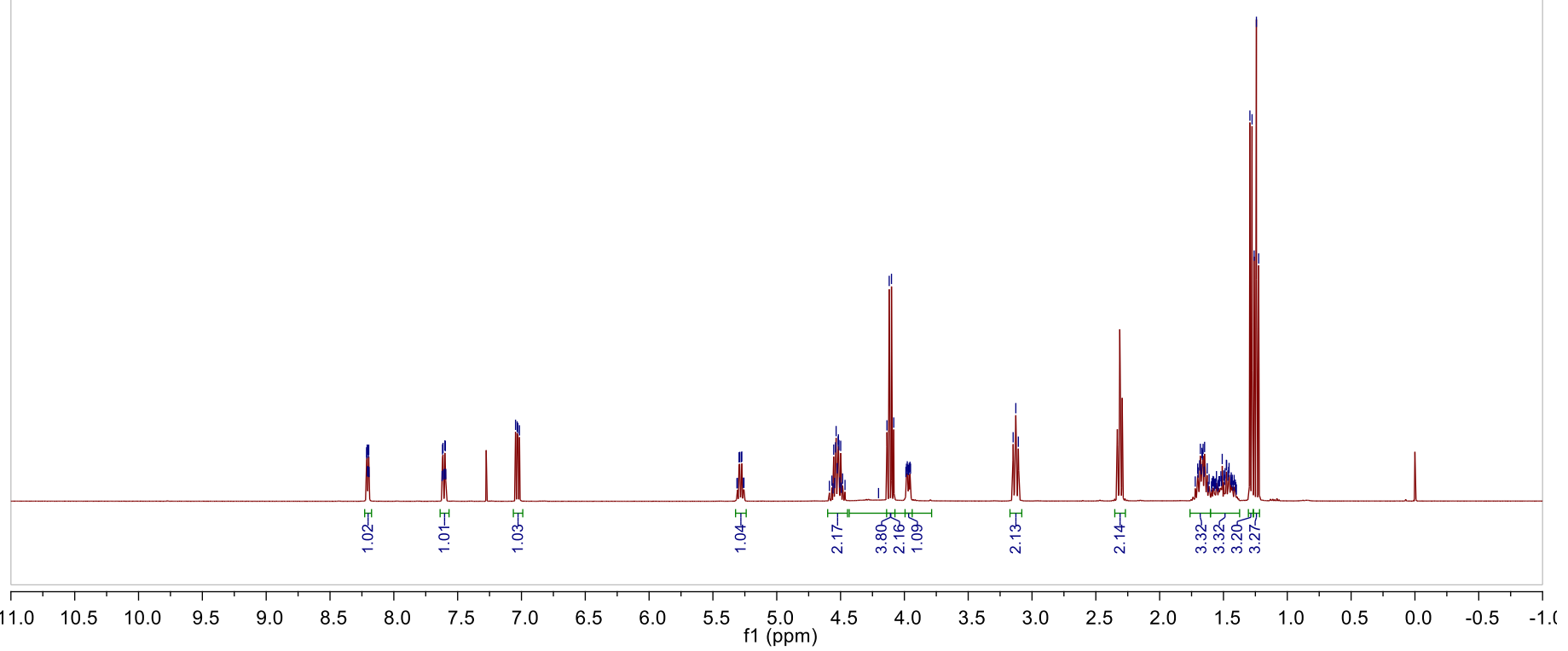<smiles>CCOC(=O)CCCCC(O)C(=S)N1CCc2cccnc21</smiles>

${ }^{13} \mathrm{C}$ NMR: $\mathbf{5 f}$

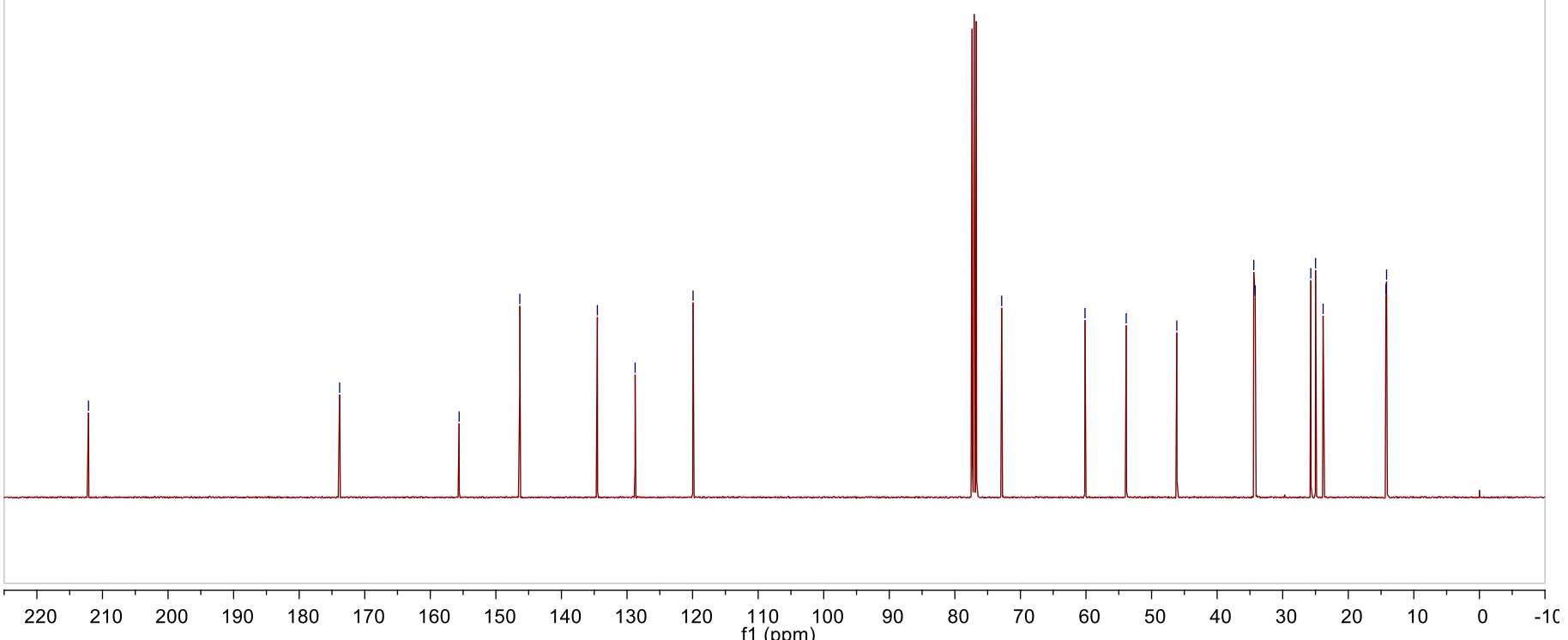




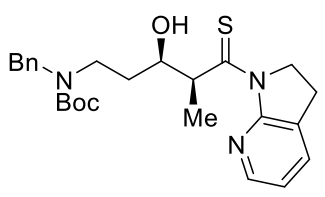

${ }^{1} \mathrm{H}$ NMR: $5 \mathbf{g}$

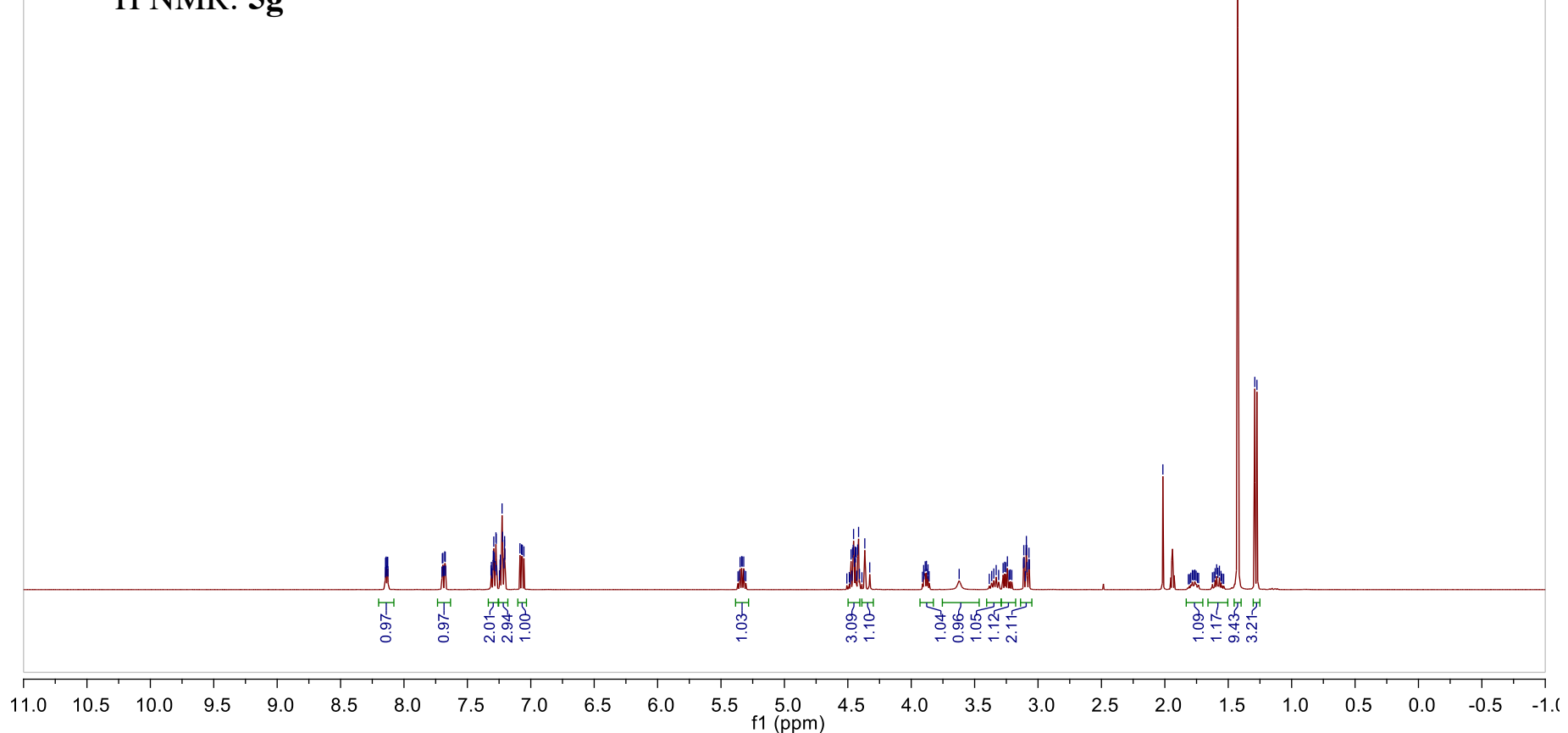

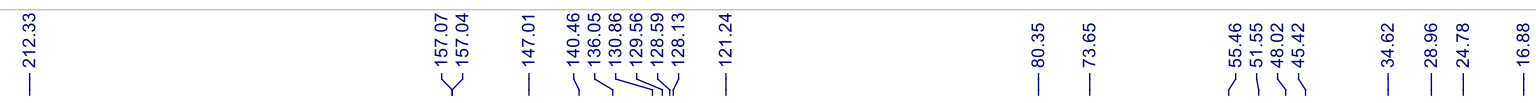

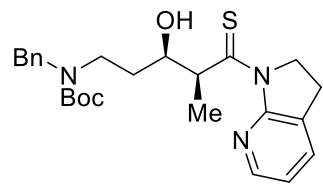

${ }^{13}$ C NMR: 5g

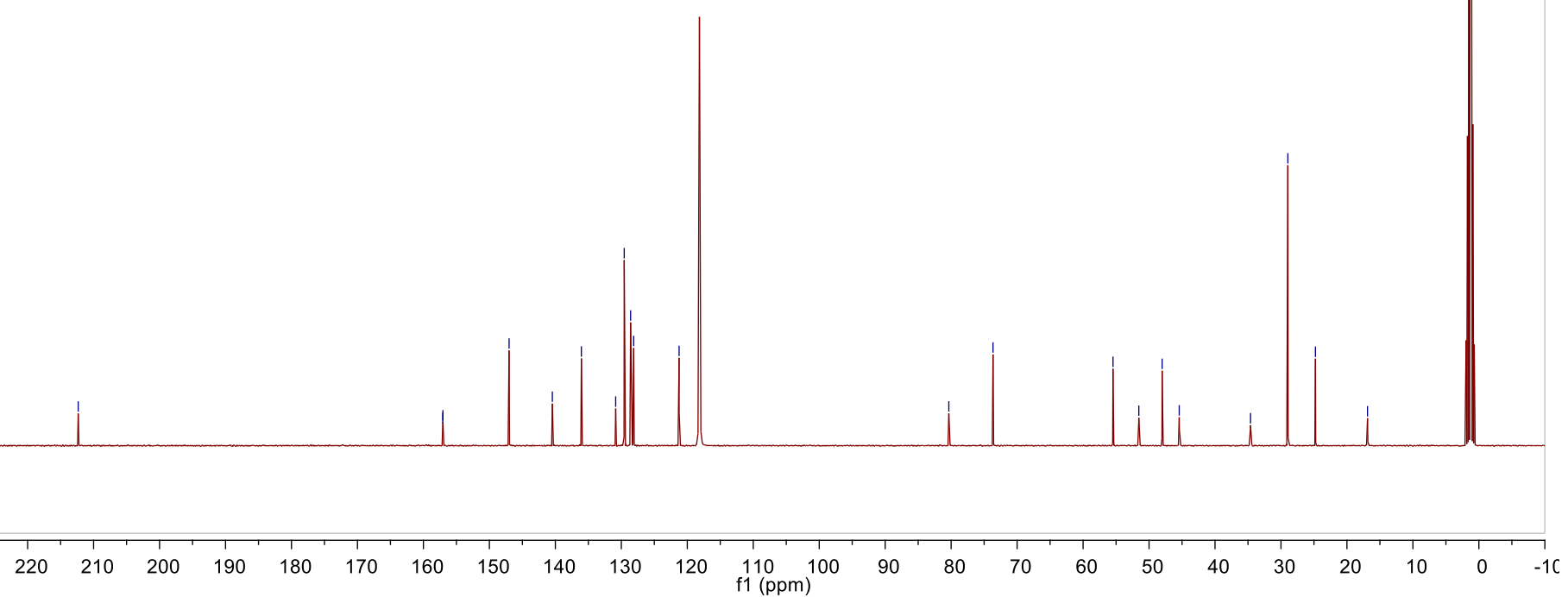




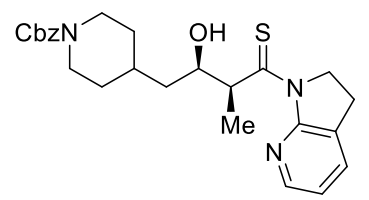

${ }^{1} \mathrm{H}$ NMR: $\mathbf{5 h}$
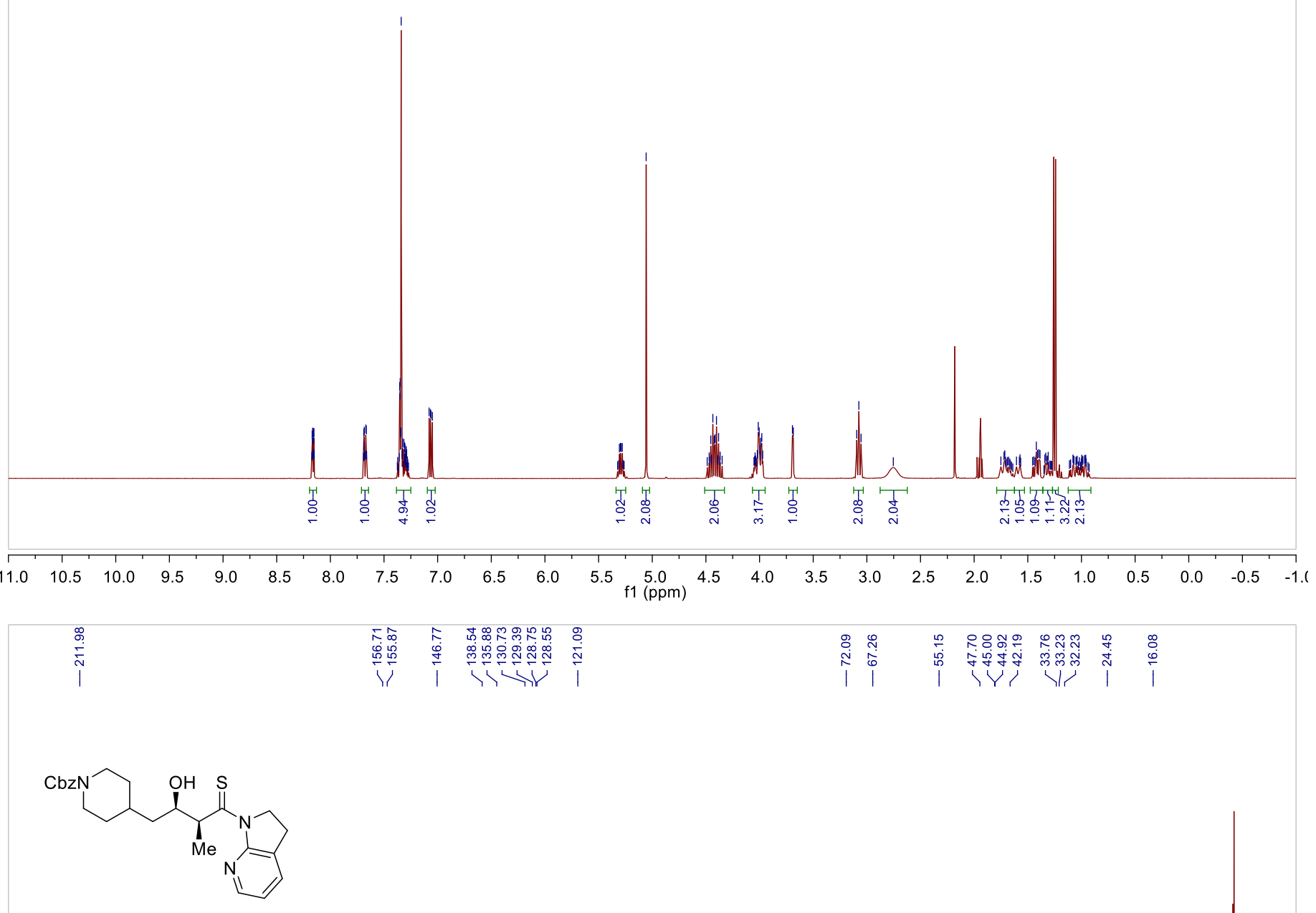

${ }^{13} \mathrm{C}$ NMR: $\mathbf{5 h}$
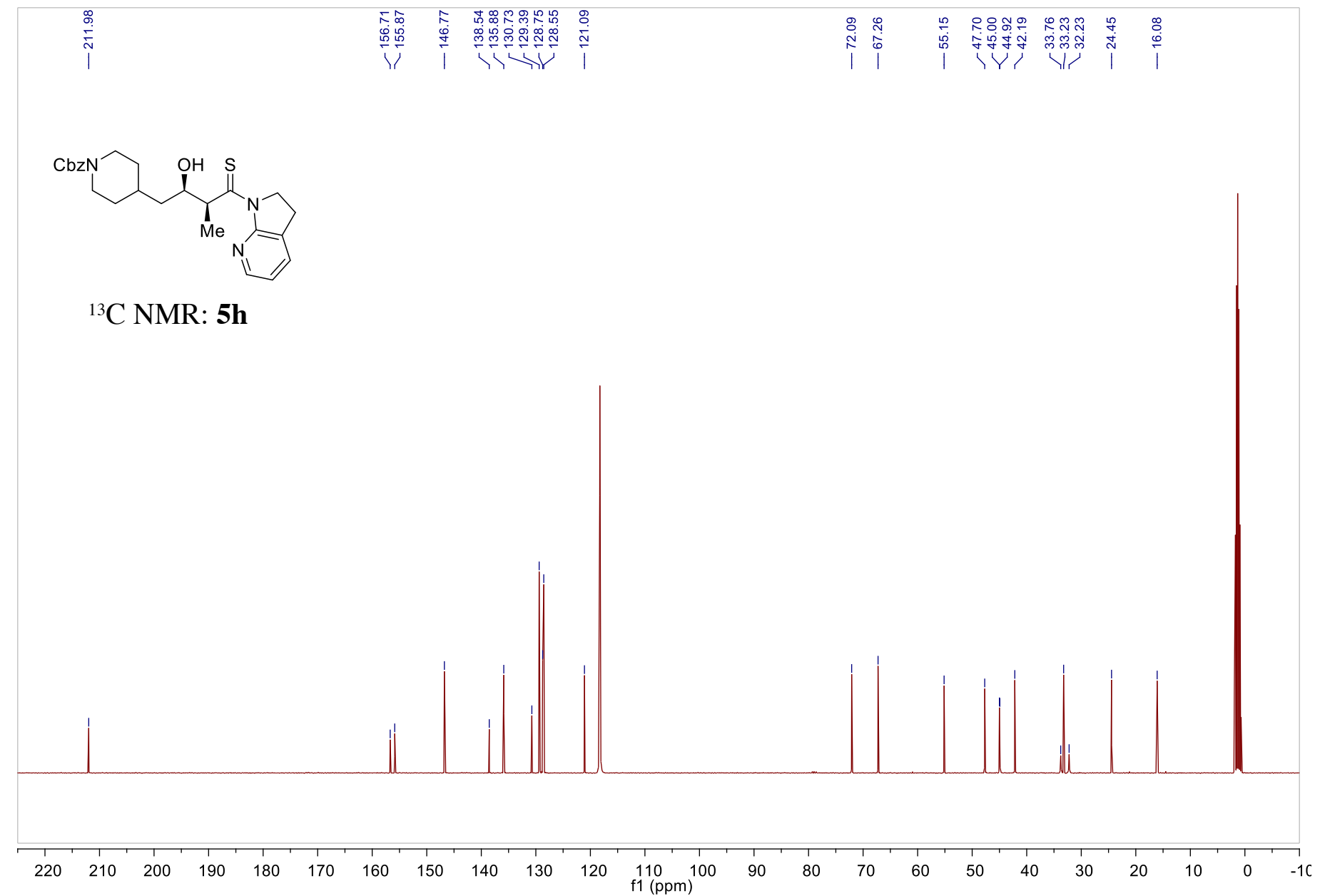


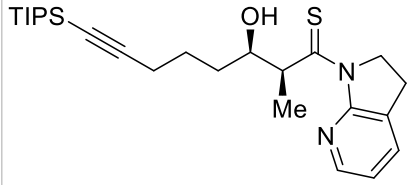

${ }^{1} \mathrm{H}$ NMR: $5 \mathbf{i}$
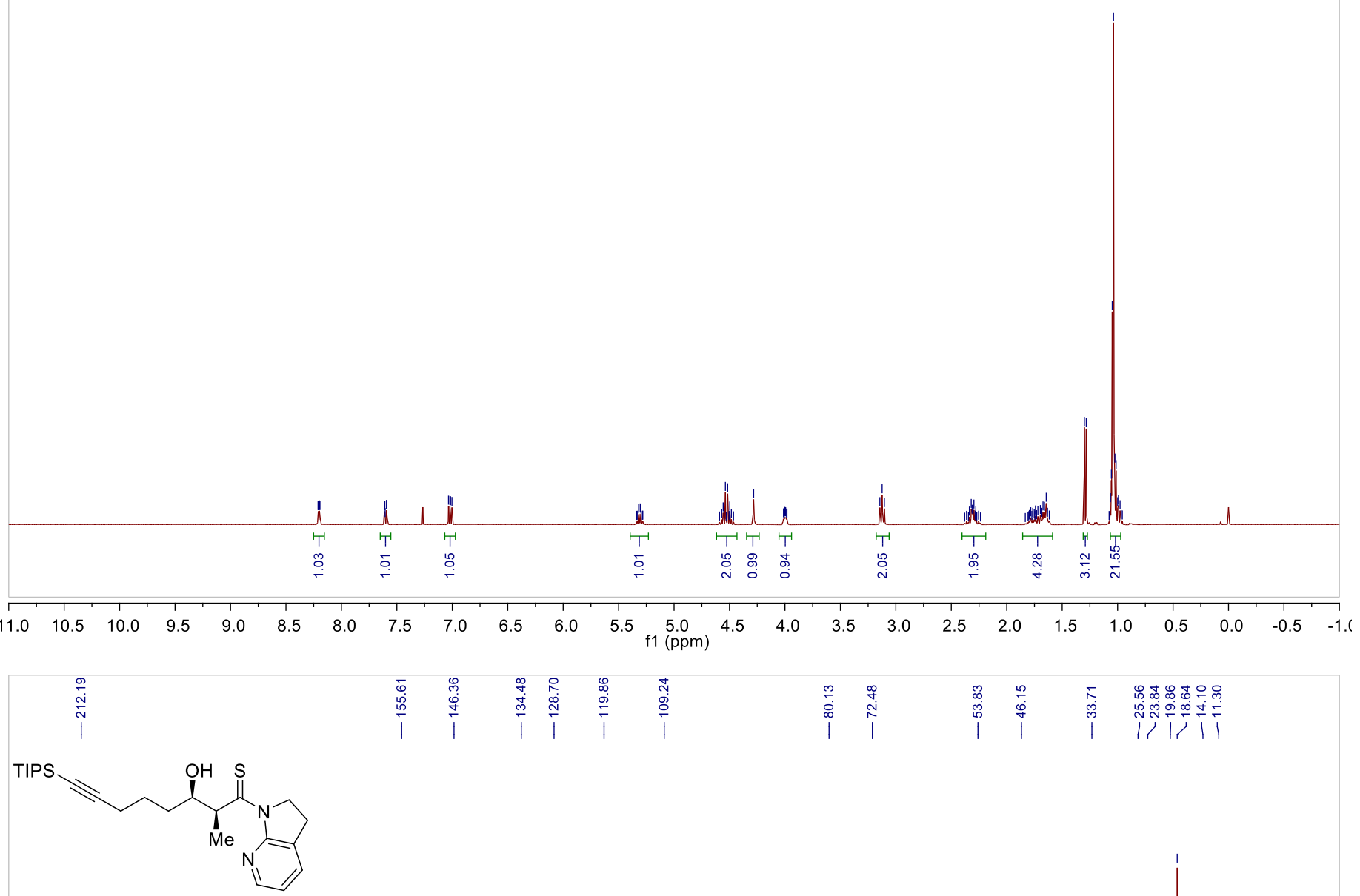

${ }^{13}$ C NMR: 5i

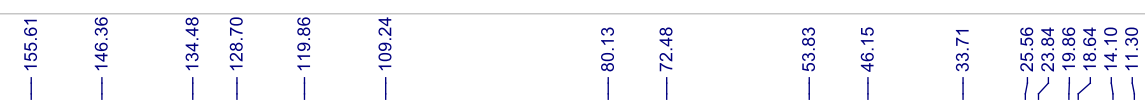

\section{CNMR: 5 i}

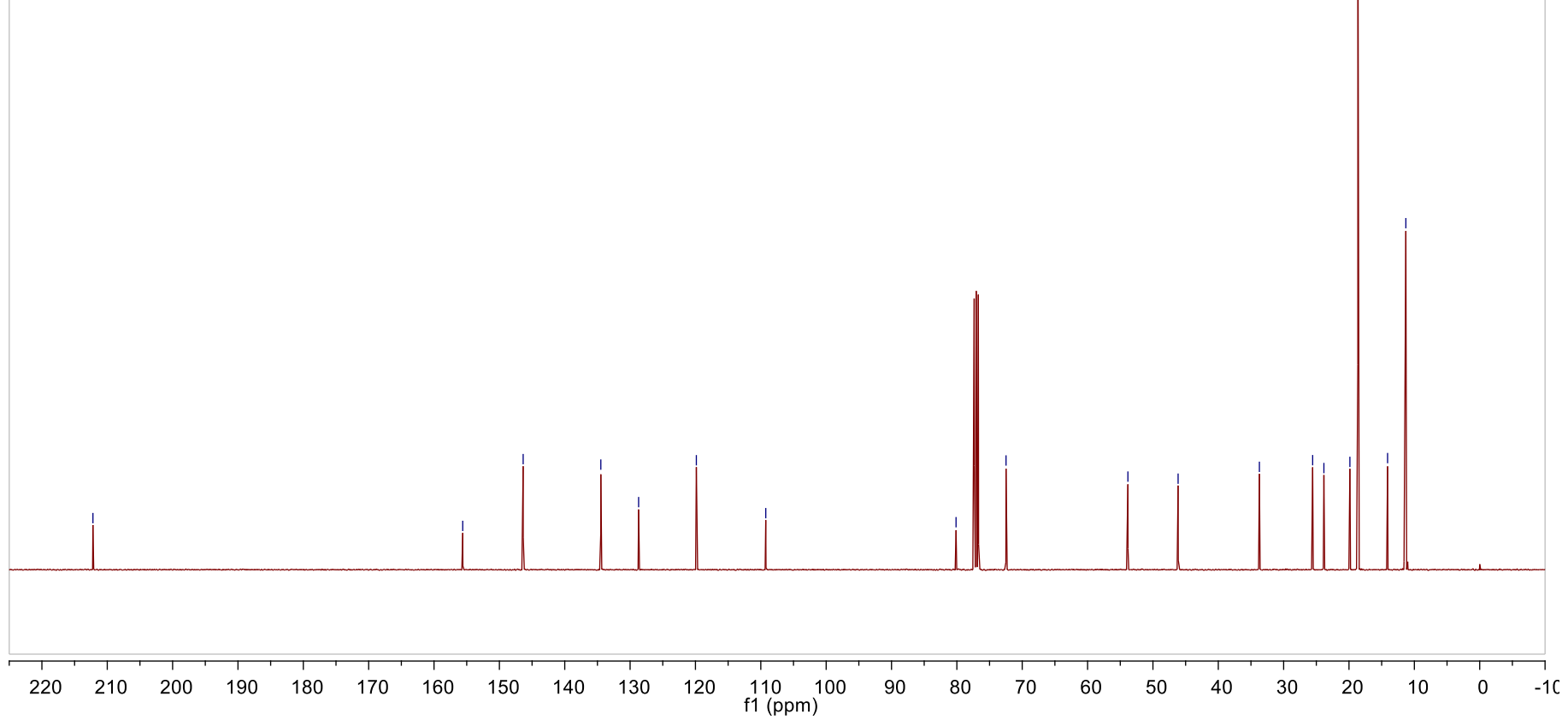


<smiles>C[C@H](CCC1CCSCC1)[C@@H](O)C(=S)N1CCc2cccnc21</smiles>

${ }^{1} \mathrm{H}$ NMR: 5j
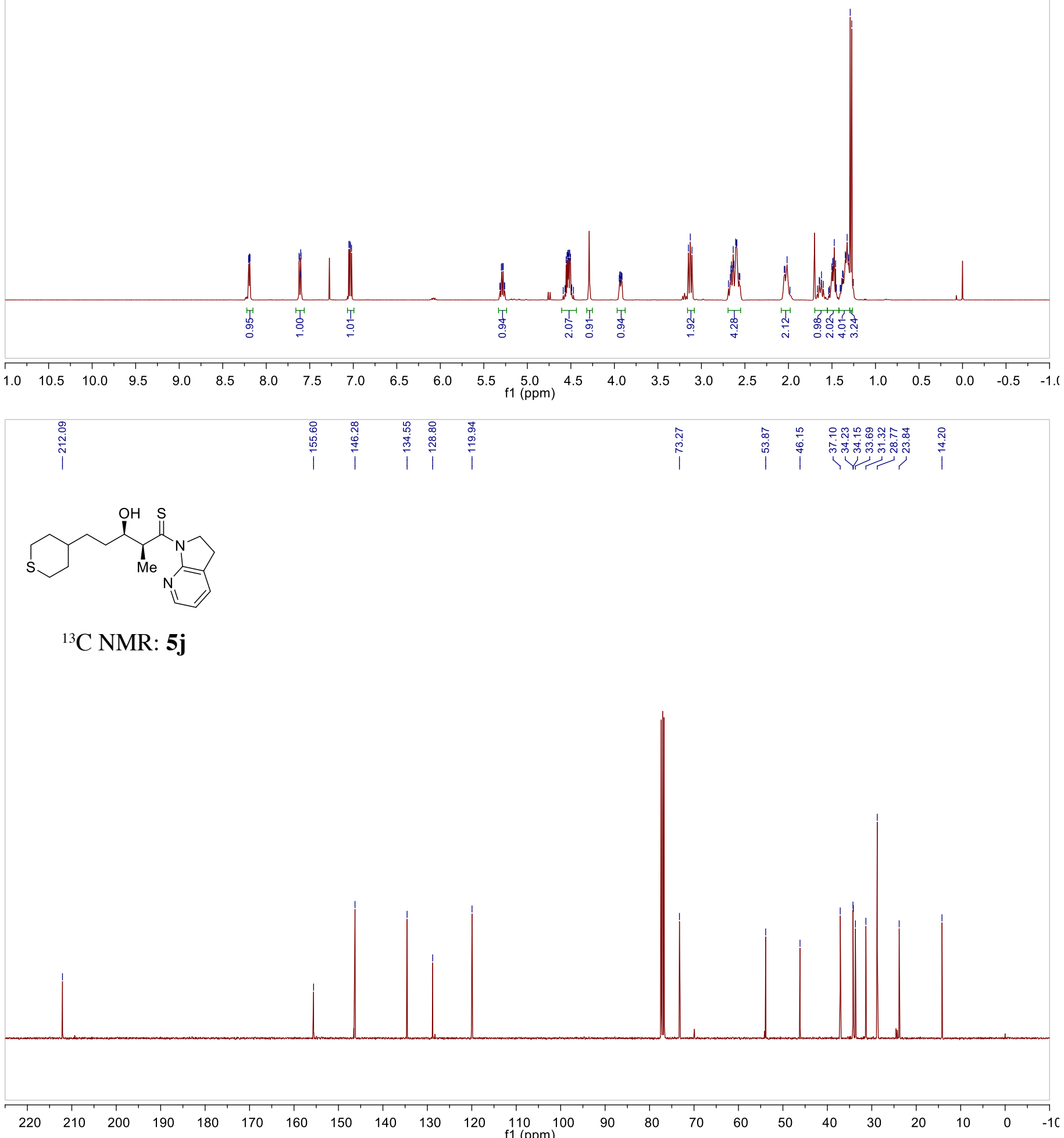
<smiles>CCC(C(=S)N1CCc2cccnc21)C(O)CCc1ccccc1</smiles>

${ }^{1} \mathrm{H}$ NMR: 5k
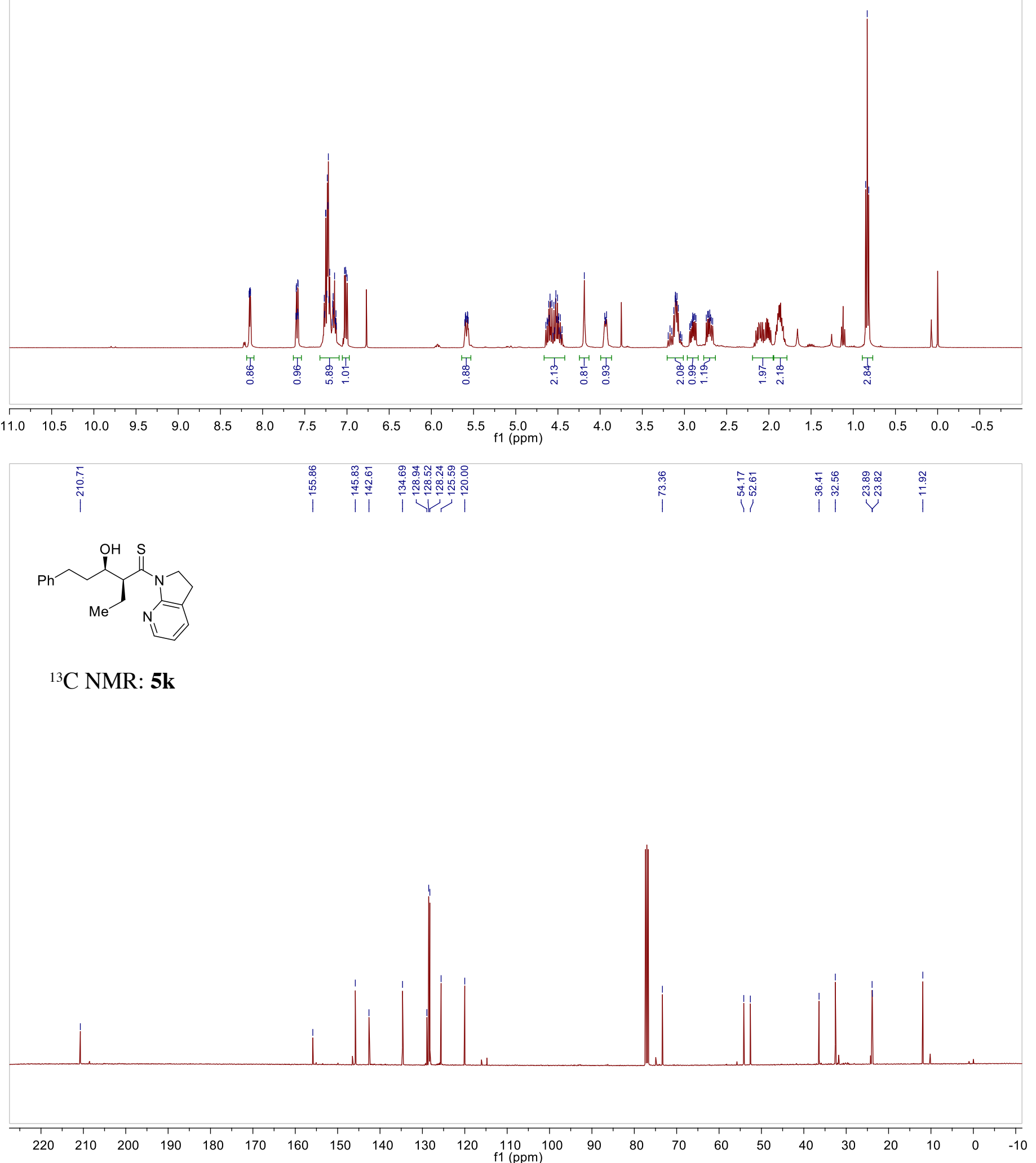
<smiles>O[C@H](CCc1ccccc1)[C@H](Cc1ccccc1)C(=S)N1CCc2cccnc21</smiles>

${ }^{1} \mathrm{H}$ NMR: $5 \mathbf{l}$

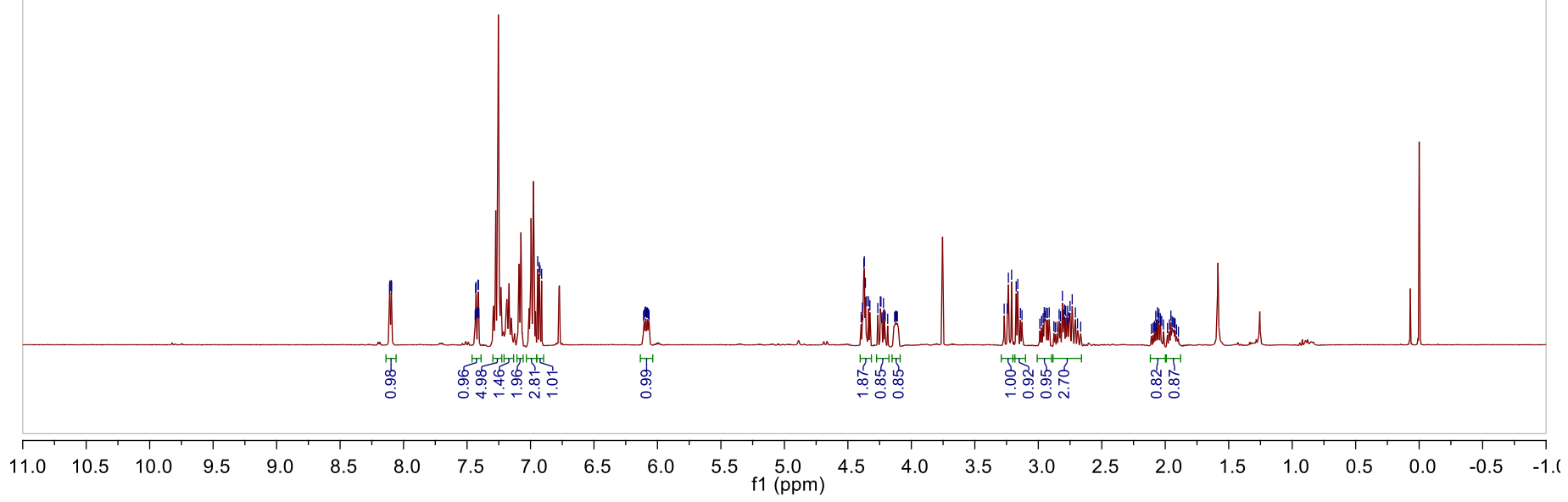<smiles>OC(CCc1ccccc1)C(Cc1ccccc1)C(=S)N1CCc2cccnc21</smiles>

${ }^{13}$ C NMR: 5 l

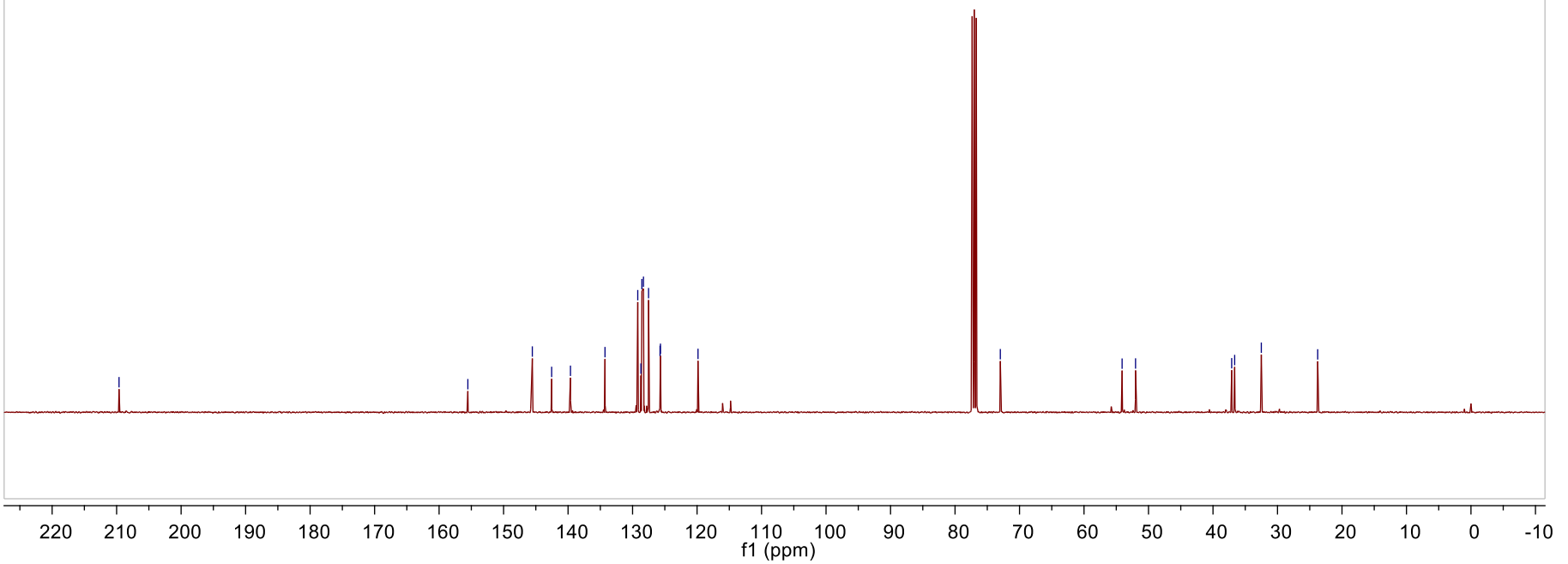


<smiles>O[C@H](CCc1ccccc1)[C@H](COc1ccccc1)C(=S)N1CCc2cccnc21</smiles>

${ }^{1} \mathrm{H}$ NMR: 5m
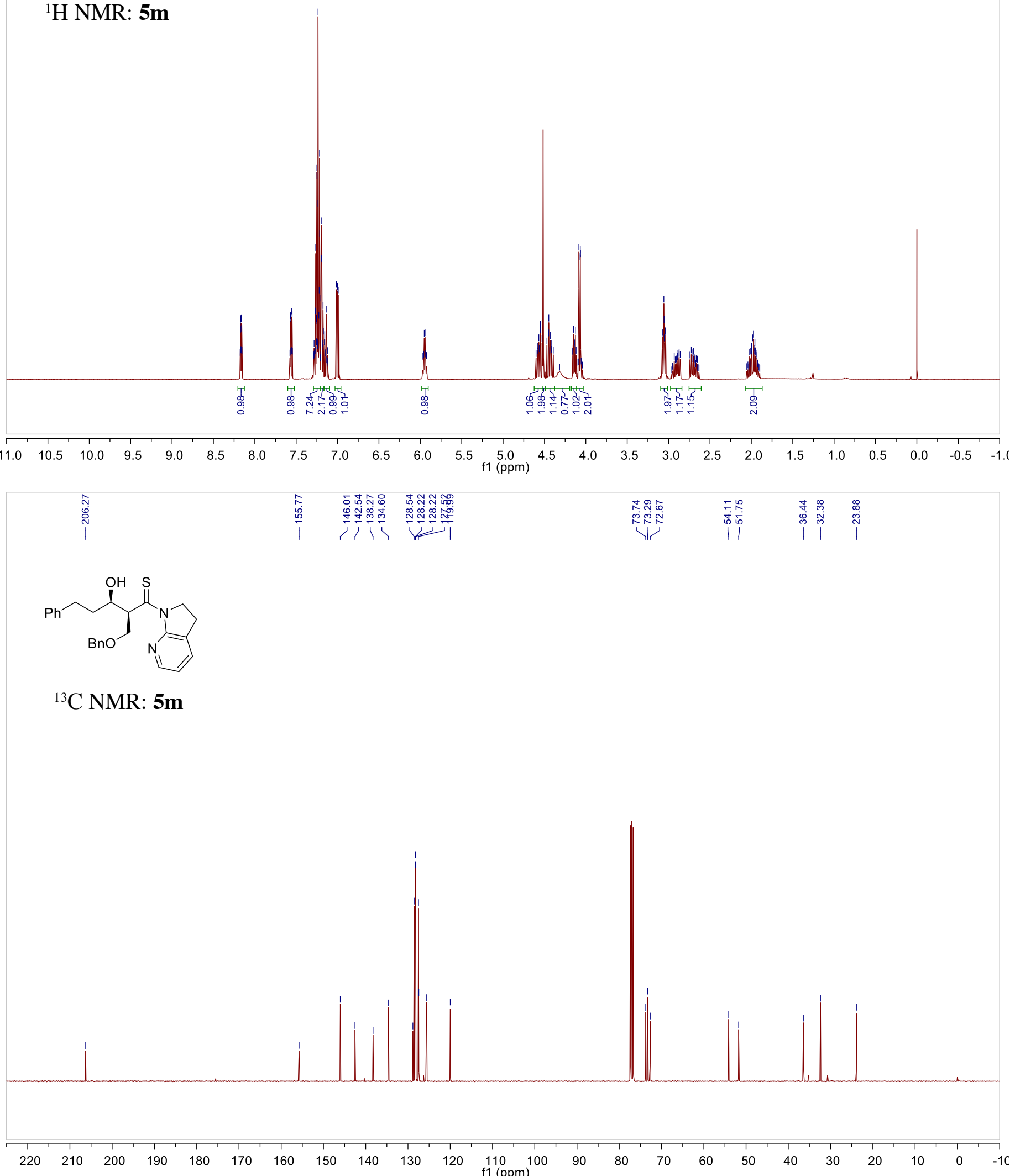


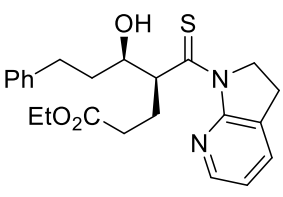

${ }^{1} \mathrm{H}$ NMR: 5n
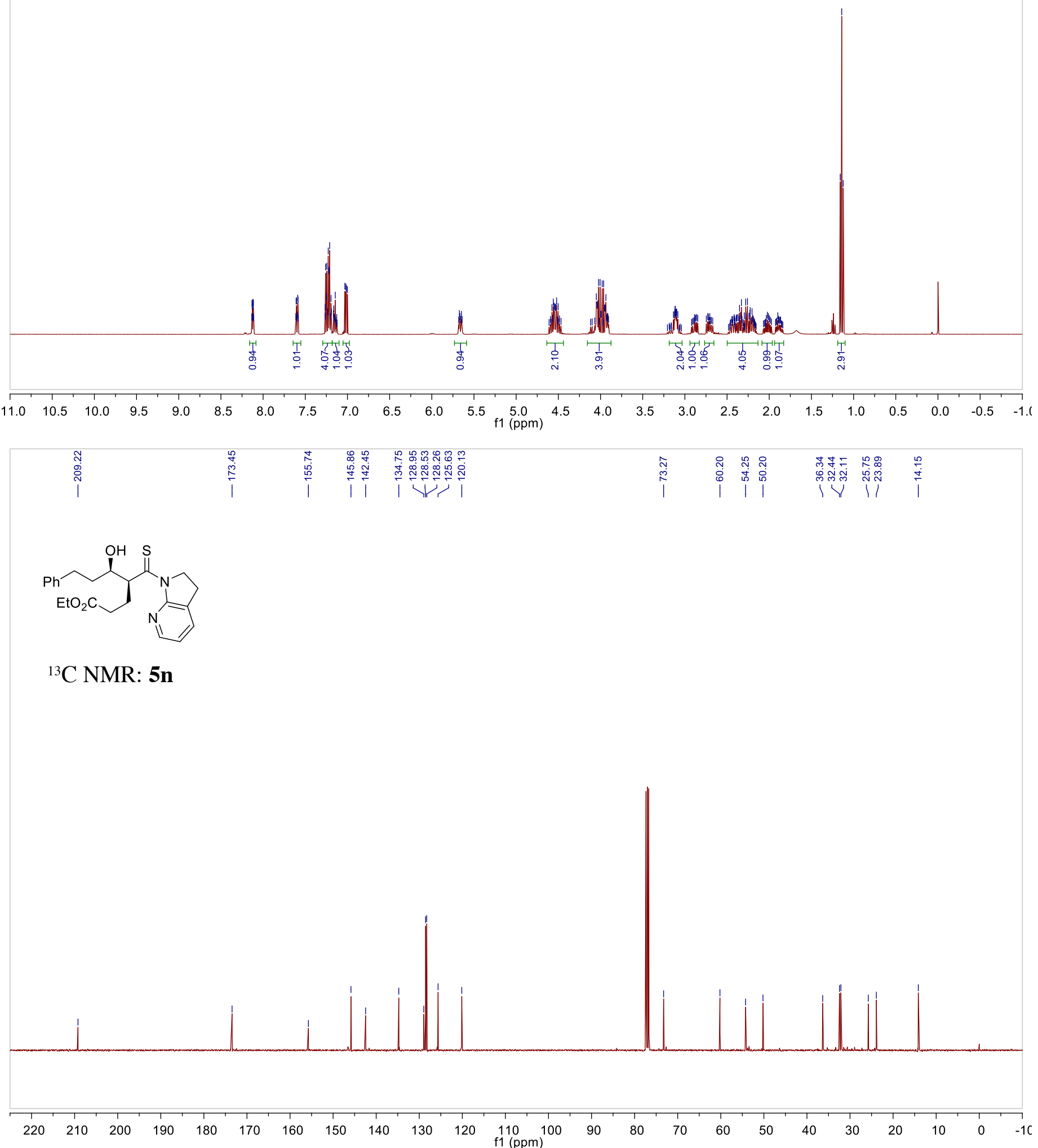
<smiles>OC(CCc1ccccc1)C(CC(F)(F)F)C(=S)N1CCc2cccnc21</smiles>

${ }^{1} \mathrm{H}$ NMR: 50
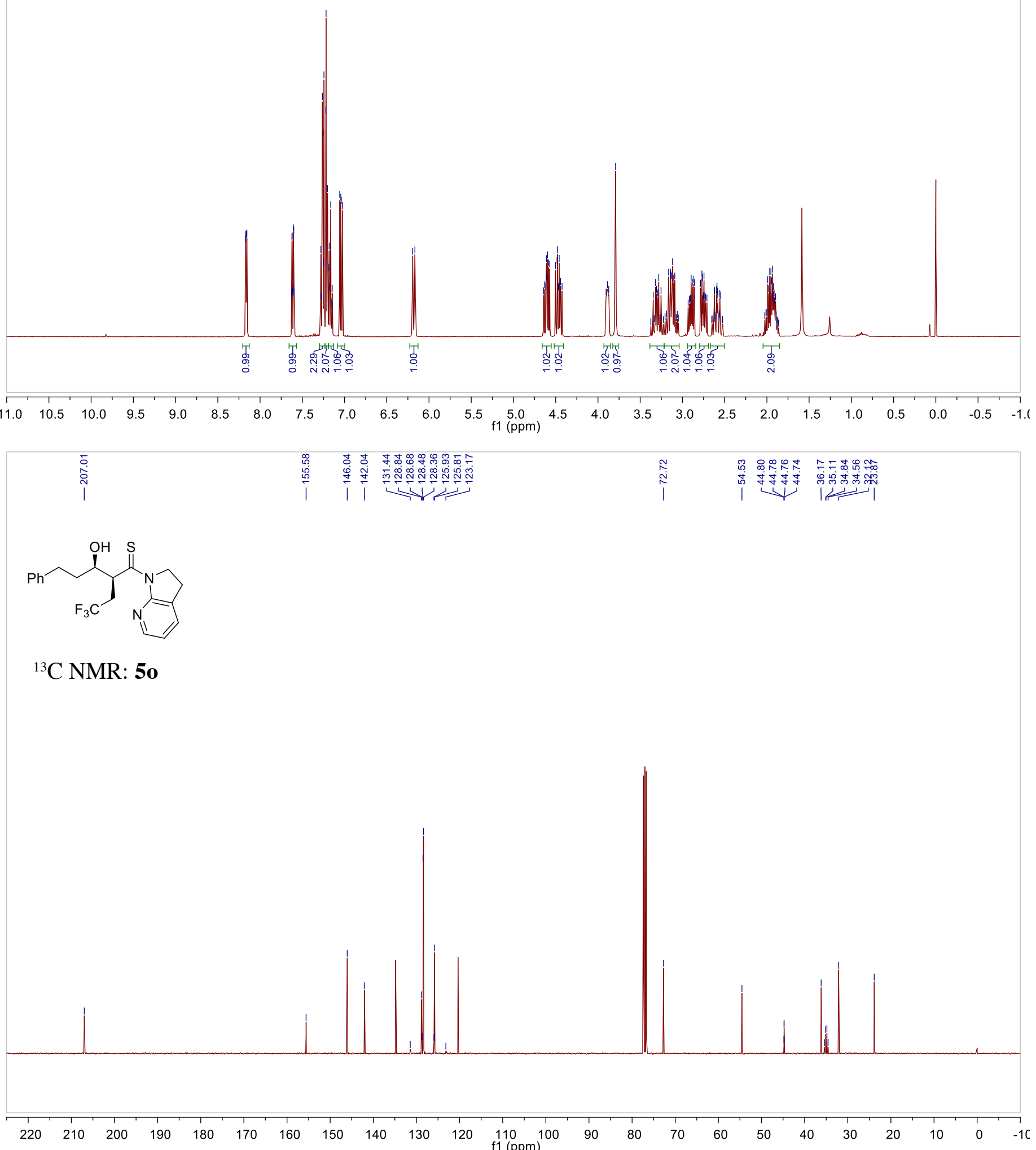


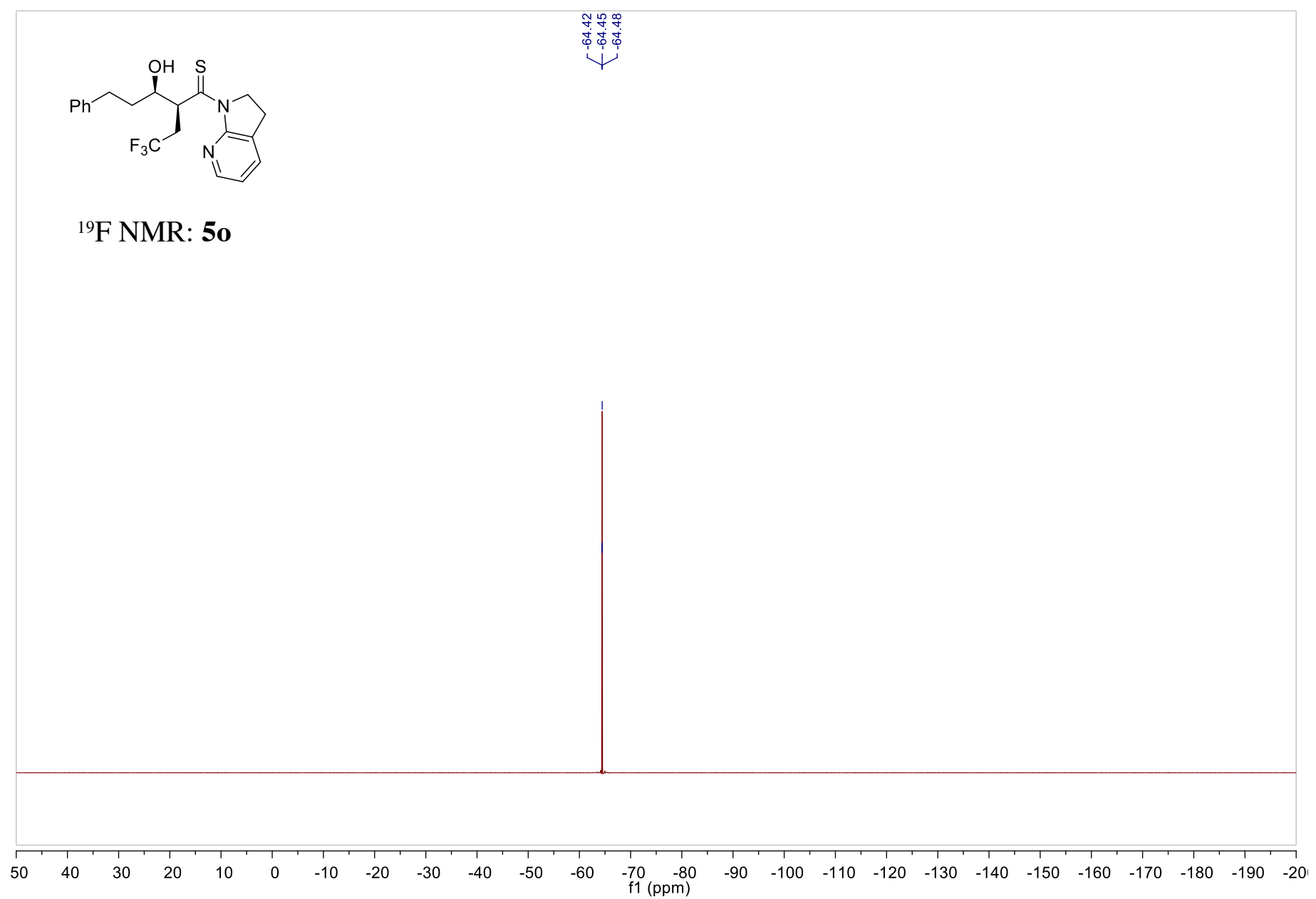


<smiles>CC(C)=CCC[C@H](C)C[C@H](O)C(C)C(=S)N1CCc2cccnc21</smiles>

${ }^{1} \mathrm{H}$ NMR: 5p
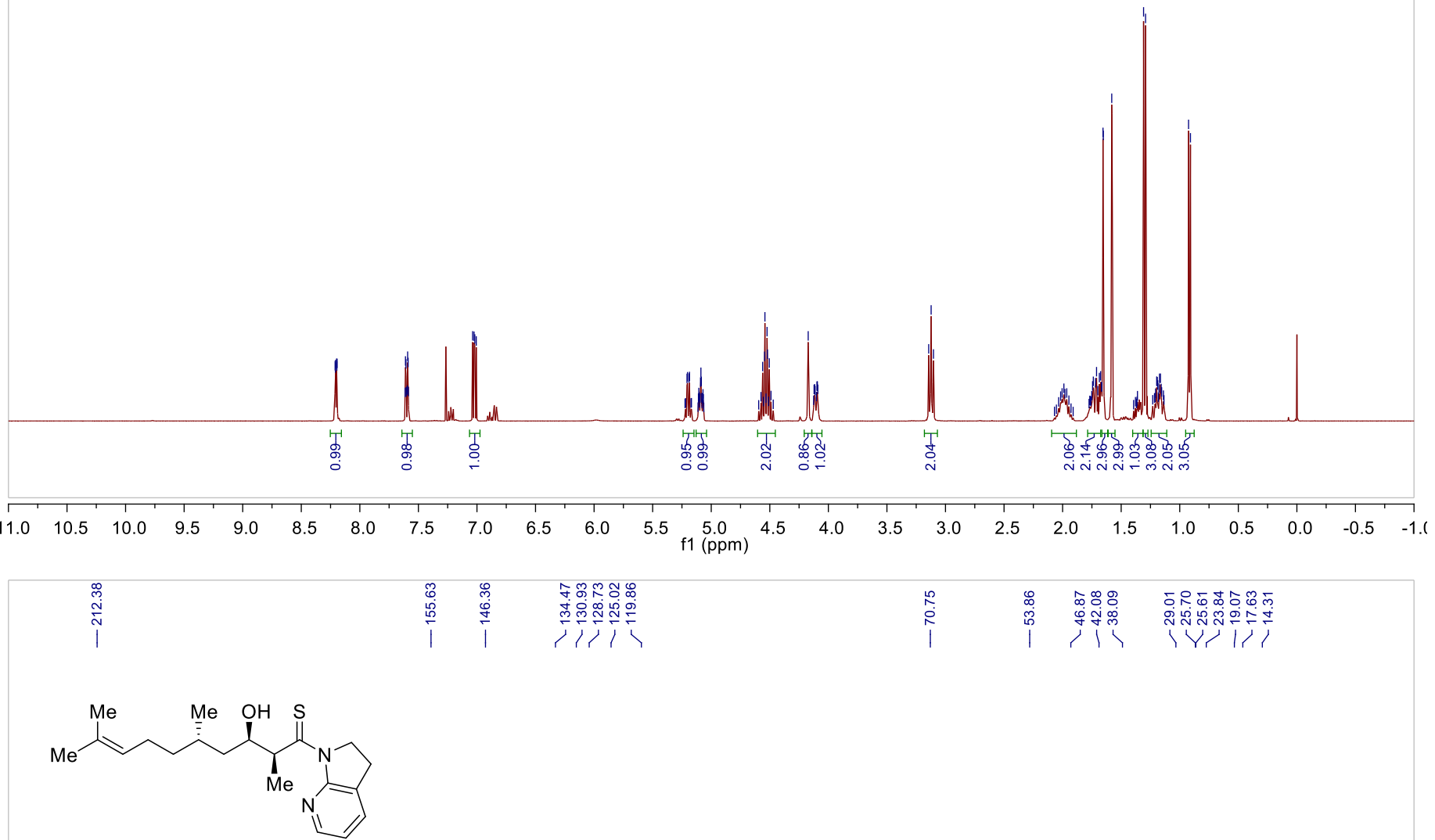

${ }^{13}$ C NMR: 5p

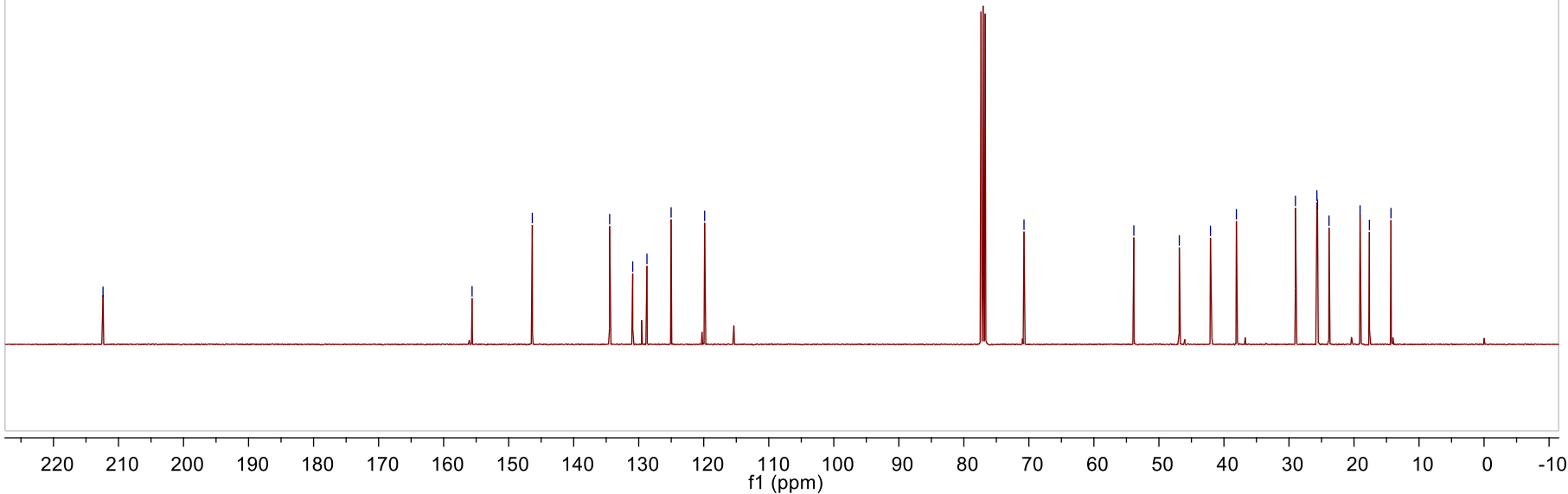




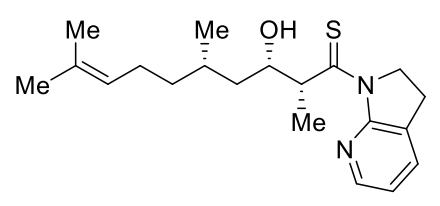

${ }^{1} \mathrm{H}$ NMR: 5q
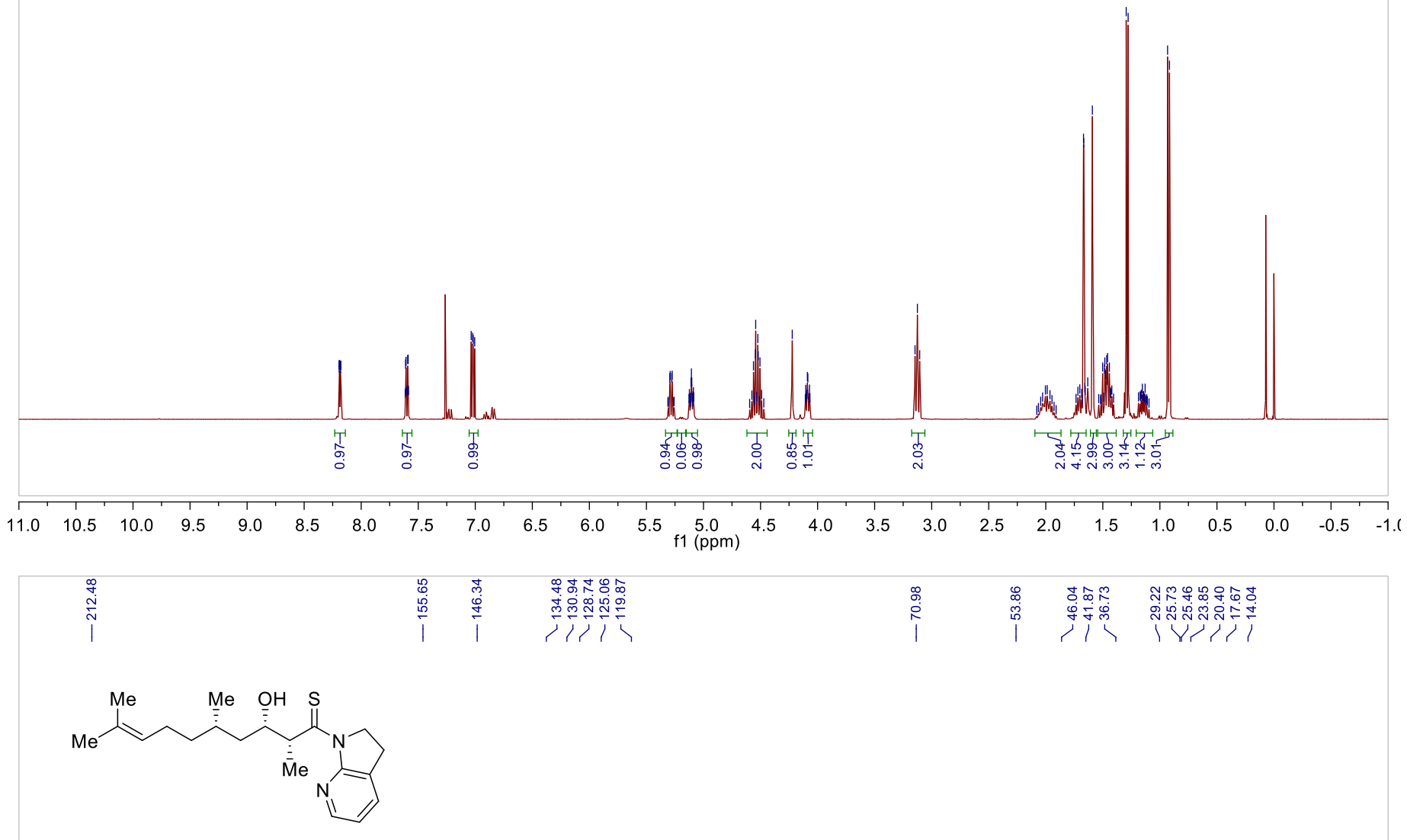

${ }^{13}$ C NMR: 5q

I

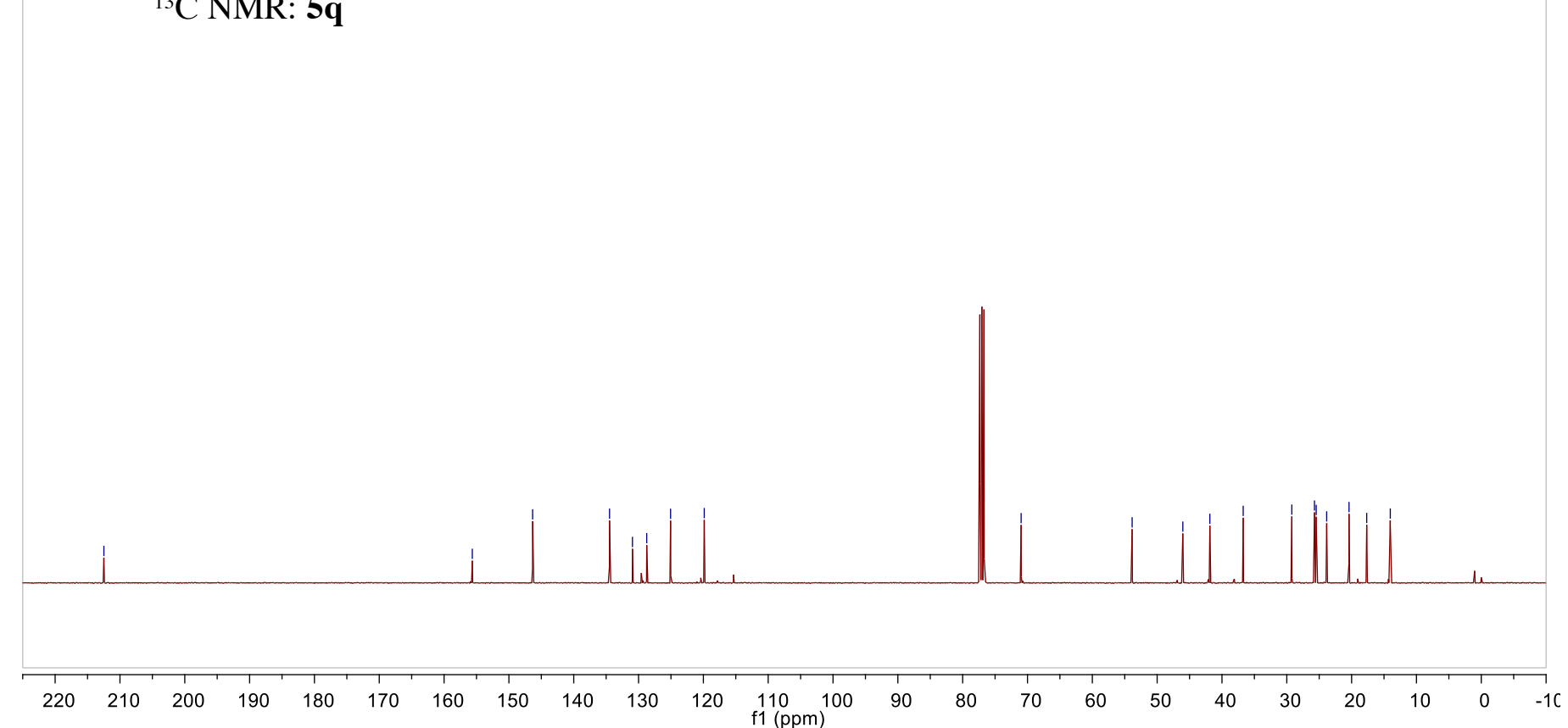



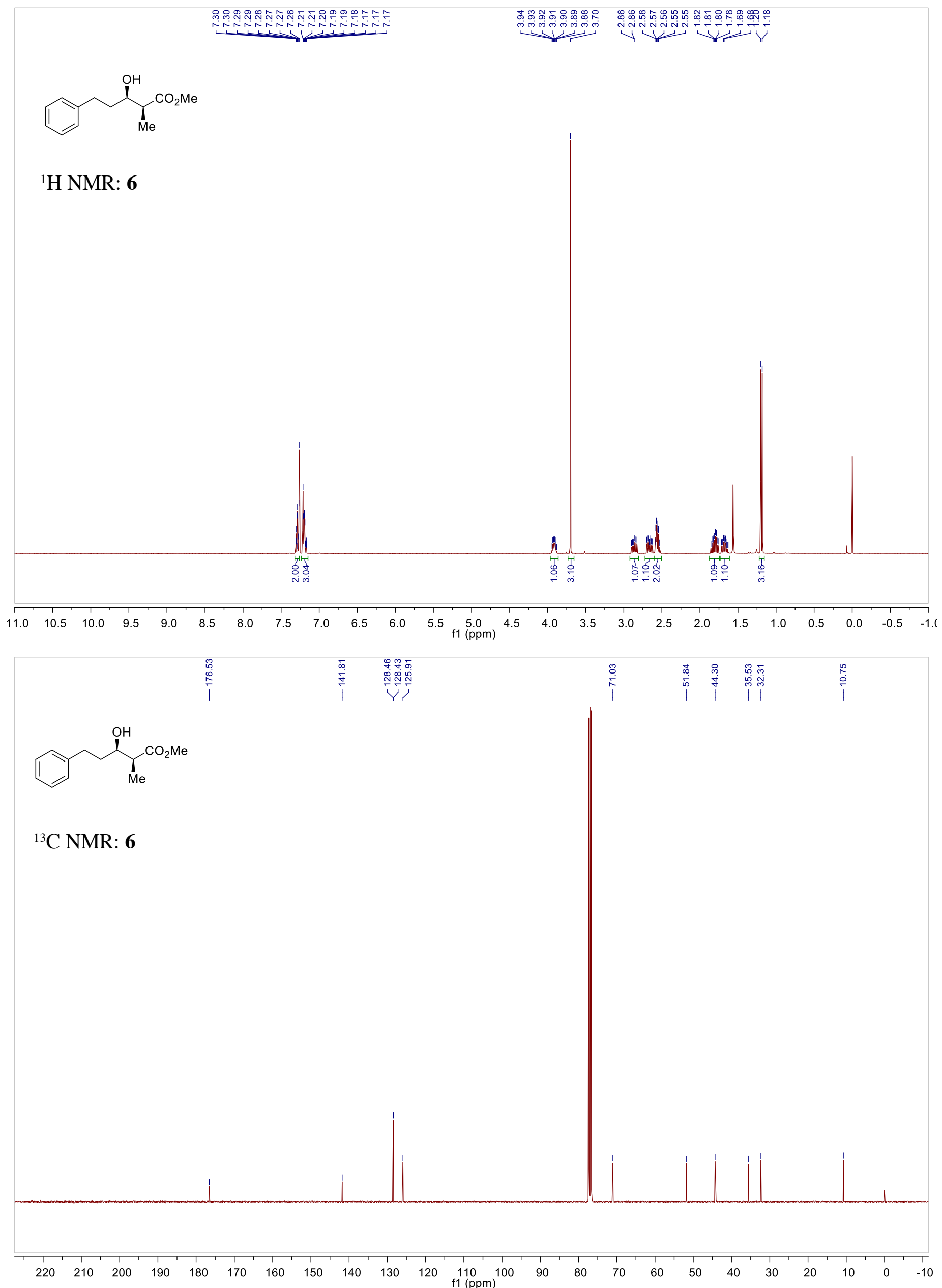

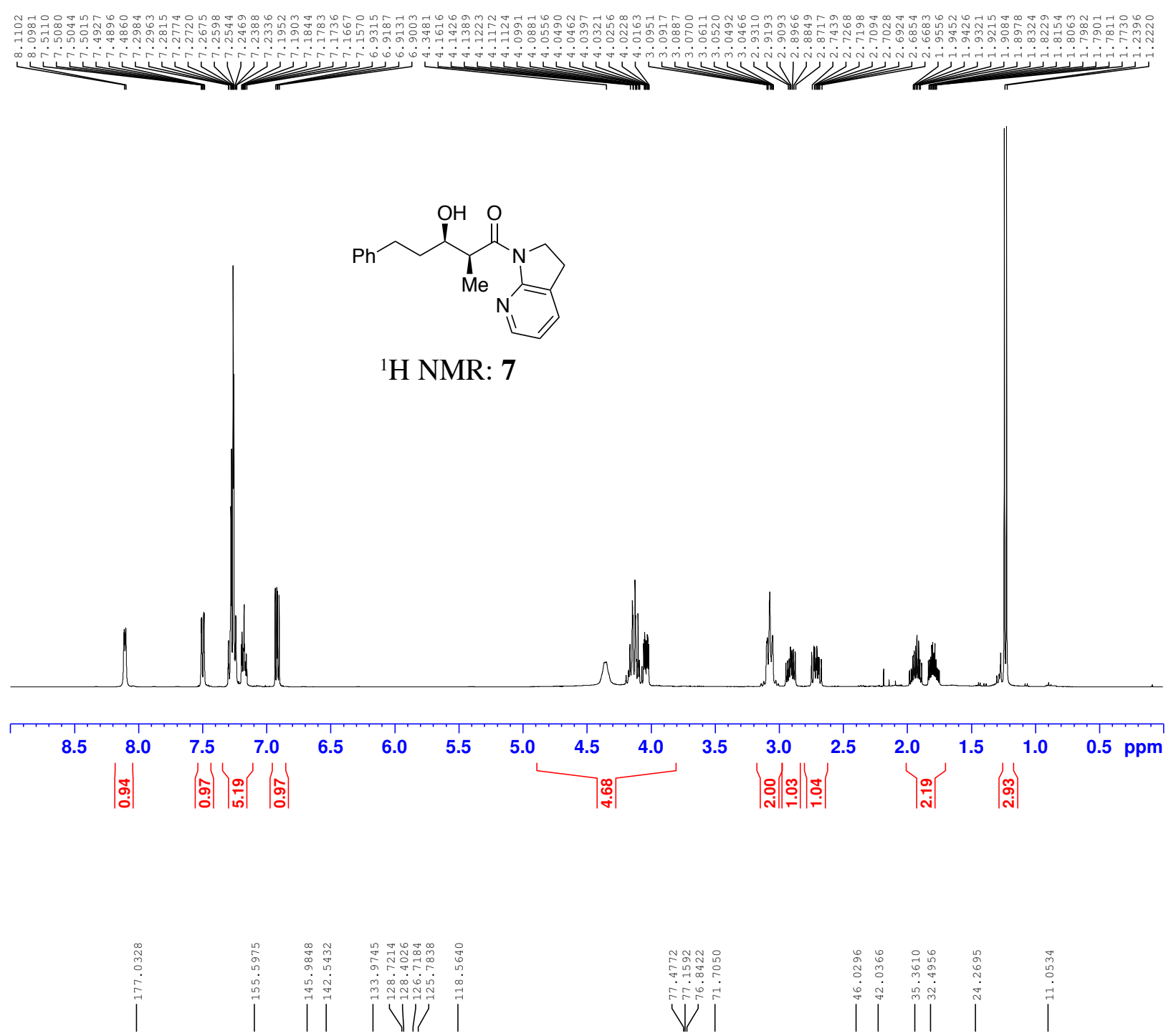<smiles>[M]C(C(=O)N1CCc2cccnc21)C(O)CCc1ccccc1</smiles>

${ }^{13} \mathrm{C}$ NMR: 7

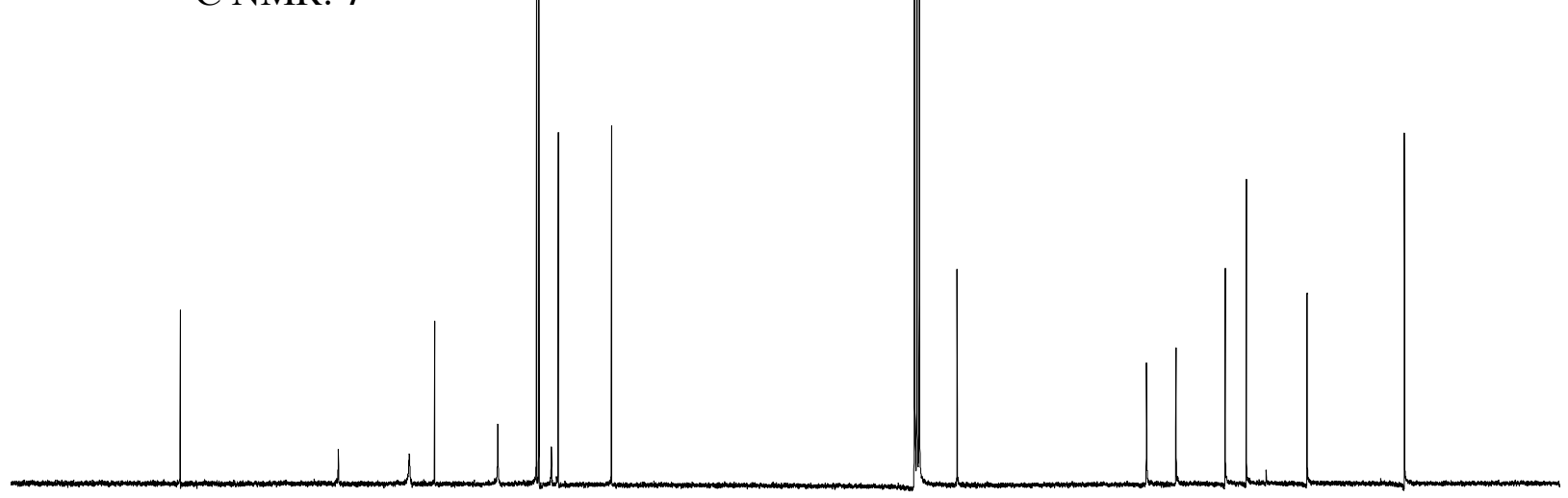

$\begin{array}{llllllllllllllllllll}190 & 180 & 170 & 160 & 150 & 140 & 130 & 120 & 110 & 100 & 90 & 80 & 70 & 60 & 50 & 40 & 30 & 20 & 10 & 0\end{array}$ 